\title{
Annual dialysis data report 2017, JSDT Renal Data Registry
}

\author{
Kosaku Nitta', Ikuto Masakane ${ }^{1,2^{*}}$, Norio Hanafusa', Masatomo Taniguchi', Takeshi Hasegawa', Shigeru Nakai', \\ Shunsuke Goto', Atsushi Wada', Takayuki Hamano', Junichi Hoshino', Nobuhiko Joki', Masaki Abe', \\ Keichi Yamamoto ${ }^{1}$, Hidetomo Nakamoto ${ }^{1}$ and on behalf of Japanese Society for Dialysis Therapy Renal Data \\ Registry Committee
}

\begin{abstract}
The annual survey of the Japanese Society for Dialysis Therapy Renal Data Registry (JRDR) was conducted for 4413 dialysis facilities at the end of 2017; among which 4360 facilities (98.8\%) responded to the facility questionnaire, and 4188 (94.9\%) responded to the patient questionnaire. The response rate of the 2017 survey was comparable with the past, even though it was the third year after the new anonymization method. The number of chronic dialysis patients in Japan continues to increase every year; it has reached 334,505 at the end of 2017. The mean age was 68.43 years. The prevalence rate was 2640 patients per million population. Diabetic nephropathy was the most common primary disease among the prevalent dialysis patients (39.0\%), followed by chronic glomerulonephritis (27.8\%) and nephrosclerosis (10.3\%). The rate of diabetic nephropathy and nephrosclerosis has been increasing year by year, whereas that of chronic glomerulonephritis was declining. The number of incident dialysis patients during 2017 was 40,959; it has remained stable since 2008. The average age was 69.68 years and diabetic nephropathy $(42.5 \%)$ was the most common cause in the incident dialysis patients. These patients caused by diabetes did not change in number for recent several years. Further, 32,532 patients died in 2017; the crude mortality rate was 9.8\%. The patients treated by hemodiafiltration (HDF) have been increasing rapidly from the revision of medical reimbursement for HDF therapy in 2012. It has attained 95,140 patients at the end of 2017, which were 18,304 greater than that in 2016. The number of peritoneal dialysis (PD) patients was 9090 in 2017, which had been slightly decreasing since 2014. Further, $19.4 \%$ of PD patients treated in the combination of hemodialysis (HD) or HDF therapy (hybrid therapy). And 984 patients were treated by home HD therapy at the end of 2017; it increased by 49 from 2016.
\end{abstract}

Trial registration: JRDR was approved by the ethical committee of JSDT (approval number 1-3) and has been registered in "University hospital Medical Information Network (UMIN) Clinical Trials Registry" as a clinical trial ID of UMIN000018641 at 8th August 2015. https://upload.umin.ac.jp/cgi-bin/ctr/ctr_view_reg.cgi?recptno=R000021578 (Accessed 31 July 2019).

\footnotetext{
*Correspondence: imasakan.aipod@seieig.or.jp

This article is translated from Japanese, originally published as "Annual dialysis data report 2017, JSDT Registry" in the Journal of the Japanese Society for Dialysis Therapy, 2018; 51: 699-766 with permission from Japanese Society for Dialysis Therapy. The original work is at "https://doi.org/ 10.4009/jsdt.51.699" (Accessed 31 July 2019).

${ }^{1}$ Japanese Society for Dialysis Therapy Renal Data Registry Committee, Japanese Society for Dialysis Therapy, Tokyo, Japan

${ }^{2}$ Department of Nephrology, Yabuki Hosipital, 4-5-5 Shimakita, Yamagata City, Yamagata 990-00885, Japan
}

\section{Part I. JRDR 2017 annual data report: general remarks Introduction}

Since 1968, the Japanese Society for Dialysis Therapy (JSDT) has conducted a survey of the status of chronic dialysis treatment in Japan at the end of every year. This survey, known as the JSDT Renal Data Registry (JRDR), covers nearly all dialysis facilities throughout the country $[1,2]$. Although participating facilities are not compensated, the nearly complete response rate ensures that it is an unbiased survey of the status of regular dialysis in

(c) The Author(s). 2019 Open Access This article is distributed under the terms of the Creative Commons Attribution 4.0 International License (http://creativecommons.org/licenses/by/4.0/), which permits unrestricted use, distribution, and reproduction in any medium, provided you give appropriate credit to the original author(s) and the source, provide a link to the Creative Commons license, and indicate if changes were made. The Creative Commons Public Domain Dedication waiver (http://creativecommons.org/publicdomain/zero/1.0/) applies to the data made available in this article, unless otherwise stated. 
Japan. It is, therefore, rare in the world. On the publication of the latest survey, the authors would like to express their sincere gratitude to all who participated in this survey while performing their routine clinical tasks at the same time.

JRDR had previously included two types of reports: $A n$ Overview of Regular Dialysis Treatment in Japan, the Illustrated Report and An Overview of Regular Dialysis Treatment in Japan, the CD-ROM Report, both of which were released at the end of the year after the target year, and the following year the reports were published in the Japanese-language edition of the Society's journal [1]. The English-language versions were then published approximately 6 months later in the English-language edition of the Society's journal, which is known as Renal Replacement Therapy (prior to the 2014 Report, it was published in the journal Therapeutic Apheresis and Dialysis). Starting in 2015, the JSDT began examining methods of reporting the results of the JRDR as the editorial policy regarding the charts and graphs listed in both the Illustrated Report and the CD-ROM Report was largely unified. As a result, in 2017, the survey was produced in full color for the first time. This was then published in the December 2018 issue of the Japanese-language journal and the Illustrated Report was discontinued. Previously, the Illustrated Report had been distributed to JSDT member dialysis facilities as well as facilities that participated in the survey in printed form only. Since the change, however, the annual report-which contains almost the same information as the Illustrated Report-is now sent to all facilities and individuals who are JSDT members. We anticipate that this will lead to more effective use of the JRDR survey results. In addition, JSDT set up its Web-based Analysis of Dialysis Data Archives system (WADDA system), which allows forms to be output freely using conditions set by the user. This system has made it far more convenient for JSDT members to utilize JRDR survey results and it has allowed members to perform a variety of analyses using the most up-to-date data. In light of this new development, the publication of the current $A n$ Overview of Regular Dialysis Treatment in Japan, the CDROM Report is scheduled to be discontinued after the release of the 2019 Survey Results Report.

In the 2017, JRDR details of the prescriptions for highperformance membranes (HPM) and hemodiafiltration (HDF), which was a major feature of dialysis therapy in Japan and has been increasing rapidly in recent years, were surveyed. The similar survey was also conducted in 2008; thus, it is of significance as the latest version will show the changes in treatment technologies that have occurred over a period of approximately 10 years.

\section{Ethical basis for the JRDR survey}

In December 2014, the Ministry of Health, Labour and Welfare $(\mathrm{MHLW})$ and the Ministry of Education, Culture, Sports,
Science and Technology (MEXT) issued the Ethical Guidelines for Medical and Health Research Involving Human Subjects. This document requires all academic organizations to strictly follow ethical considerations and protect personal information [3]. JSDT adheres to these Guidelines, and as a result, starting with the survey released at the end of 2015, it strengthened its policy on anonymization and changed the survey methods it utilizes in order to improve its protection of personal information. The efforts to improve anonymization include the use of an algorithm that coverts patient information into random strings of English letters and numbers, as well as the use of a decoding key that dialysis facilities can use in their own computer systems to decode the encoded patient names, but that the administrative office of the JSDT cannot use to recreate patient information. In addition to these technology-based improvements, efforts to guarantee the ethical validity, fairness, and transparency of the survey included an examination by the ethical committee of JSDT (approval number 1-3) in March 2015, public release on the UMIN Clinical Trials Registry (UMIN000018641), and full release of these results on the JSDT homepage [4].

\section{Survey methods}

\section{Sending and recovering the questionnaires}

The JRDR annual surveys consist of two types of questionnaires: The facility survey questionnaire, which is used to investigate the number of dialysis consoles, number of staff members, number of patients, and related information, and the patient survey questionnaire, which is used to investigate data such as the dialysis prescriptions, laboratory data, and outcome factors of each patient at the dialysis facilities. For the 2017 survey, USB memory devices containing the facility surveys and 2016 anonymized patient surveys in Excel format were mailed to dialysis facilities throughout the country in December 2017. The dialysis facilities decoded the patient names using the decoding key in the USB memory device sent to them in 2015 and they then updated patient data related to patient outcomes, including survival vs. death and transfer to another facility, as well as other data. They also registered incident patients into the system. Once all patient record input and update tasks were concluded, they once again anonymized the data. After all dialysis facilities had completely anonymized the patient data, only the USB memory device containing the questionnaires was returned to the administrative office of JSDT. Paper-based patient surveying was discontinued in 2015. The initial deadline for the data was January 31, 2018, but facilities that had not returned data as of that date were encouraged to do so. To accommodate these facilities, a final deadline of June 30 was set and data collection for the end of 2017 was closed at this time.

\section{Survey items}

The following items were surveyed in 2017. 
Facility survey

1. Overview and scope of facilities

- Facility code, name of facility, and the date (month and year) that dialysis was begun at the facility

- Dialysis capabilities: simultaneous dialysis treatment capacity, maximum dialysis treatment capacity

- Number of dialysis consoles, number of consoles with endotoxin retentive filters (ETRF)

2. Patient dynamics

- Number of prevalent dialysis patients at the end of 2017 (no. of patients by treatment modality, outpatient/inpatient)

- Number of dialysis patients undergoing nightshift dialysis in 2017

- Number of incident dialysis patients in 2017 that began $\mathrm{HD}(\mathrm{F})$ and the number that began PD

- Number of deceased patients in 2017

3. Dialysis fluid quality control

- Frequency with which dialysis fluid endotoxin (ET) concentration was measured and the ET concentration

- Frequency with which dialysis fluid total viable microbial count (TVC) was measured and the TVC

- Source of dialysis water

- Frequency of residual chlorine measurement before daily dialysis practice, and the measurement technique

Awareness toward the JSDT standard for dialysis fluid (chemical contamination standard), and frequency of the measurement

\section{Patient survey}

1. Patient personal information

- Sex, date of birth, year and month of start of dialysis, year and month of transfer from another hospital, primary disease, residence (prefecture), dialysis modality, month of transfer (destination facility code), outcome category, outcome date (transfer, death, dropout, or transplantation) (destination facility code), month of death, cause of death, dates of changes, change codes, status of combined therapies involving PD with HD or HDF, etc., PD experience, and number of kidney transplants

2. HD/HDF therapy conditions
- Frequency of dialysis session per week, dialysis time per session, and blood flow rate

- HDF: dilution method, substitution fluid volume per session

- Membrane material, JSDT membrane category, membrane surface area

- Body height, pre- and post-dialysis body weight, pre-dialysis systolic blood pressure, pre-dialysis diastolic blood pressure, and pre-dialysis pulse rate

3. Laboratory findings

- Pre- and post-dialysis serum urea nitrogen (UN), preand post-dialysis serum creatinine concentration, predialysis serum albumin concentration, pre-dialysis serum C-reactive protein (CRP) concentration, predialysis serum calcium concentration, pre-dialysis serum phosphorus concentration, serum parathyroid hormone (PTH) assay method, PTH level (intact or whole $\mathrm{PTH}$ ), pre-dialysis hemoglobin concentration, serum total cholesterol concentration (total cholesterol), and serum high-density-lipoprotein-cholesterol concentration (HDL-C), pre- and post-dialysis serum beta2-microglobulin ( $\beta 2$-MG) concentration

4. Outcome factors

- Antihypertensive drug use, smoking, history of diabetes, history of myocardial infarction, history of cerebral hemorrhage, history of cerebral infarction, limb amputation, history of proximal femur fracture, history of encapsulating peritoneal sclerosis (EPS), history of carpal tunnel syndrome operation, hospitalization, cause of the hospitalization

5. Peritoneal dialysis (PD) survey

- Therapeutic history: current PD dialysis vintage, number of months in which PD was performed in 2017

- Peritoneal function: implementation of peritoneal equilibration test (PET), 4-h creatinine concentration dialysate/plasma ratio in PET (PET Cr D/P ratio)

- Dialysis prescription: type of PD fluid, volume of PD fluid per day, PD treatment time per day, daily urine volume, mean fluid removal volume per day, Kt/V by residual kidney function (residual kidney Kt/V), Kt/V by PD (PD Kt/V)

- PD method: use of automated peritoneal dialysis (APD) machine, changing maneuver of PD fluid

- PD-related infections: number of peritonitis during 2017 (peritonitis frequency), number of exit-site infections during 2017

\section{Revisions to the primary disease codes and cause of death codes}

In the 2017 survey, changes were made to the disease types and terms and the detailed primary disease codes and cause 
Table 1 Kidney disease codes for primary kidney diseases, Comparison of the new (2017 ) and the previous ( 2016), 2017

\begin{tabular}{|c|c|c|c|c|c|c|c|}
\hline \multirow{2}{*}{ Kidney disease } & \multicolumn{2}{|c|}{ Code } & \multirow{2}{*}{$\begin{array}{c}\text { Kidney disease } \\
\text { category }\end{array}$} & \multirow{2}{*}{ Kidney disease } & \multicolumn{2}{|c|}{ Code } & \multirow{2}{*}{ Classification in JRDR report } \\
\hline & \multicolumn{2}{|c|}{ Clinical diagnosis Pathologically proven } & & & Clinical diagnosis & Pathologically prover & \\
\hline Chronic glomerulonephritis & 010 & 011 & \multirow{6}{*}{\begin{tabular}{|l} 
Chronic \\
glomerulonephr \\
itis
\end{tabular}} & Chronic glomerulonephritis & 010 & 011 & \multirow{6}{*}{ Chronic glomerulonephritis } \\
\hline IgA nephropathy & 012 & 013 & & IgA nephropathy, Henoch-Schönlein purpura & 012 & 013 & \\
\hline Other proliferative glomerulonephritis & 014 & 015 & & Other proliferative glomerulonephritis & 014 & 015 & \\
\hline Membranous nephropathy & 016 & 017 & & Membranous nephropathy & 016 & 017 & \\
\hline \multirow[t]{2}{*}{ Membranoproliferative glomerulonephritis } & 018 & 019 & & Membranoproliferative glomerulonephritis & 018 & 019 & \\
\hline & & & & Focal segmental glomerulosclerosis & 240 & 241 & \\
\hline Chronic pyelonephritis & 020 & 021 & \multicolumn{2}{|c|}{ Chronic pyelonephritis } & 020 & 021 & Chronic pyelonephritis \\
\hline Other interstitial nephritis & 022 & $023^{-}$ & \multicolumn{2}{|c|}{ Interstitial nephritis } & 250 & 251 & Interstitial nephritis \\
\hline Rapidly progressive glomerulonephritis & 030 & 031 & \multicolumn{2}{|c|}{\begin{tabular}{|l|} 
Rapidly progressive glomerulonephritis (ANCA- \\
associated nephritis, anti-GBM nephritis)
\end{tabular}} & 030 & 031 & Rapidly progressive glomerulonephritis \\
\hline $\begin{array}{l}\text { Nephropathy of pregnancy/ } \\
\text { pregnancy toxemia/PIH }\end{array}$ & 050 & 051 & \multicolumn{2}{|c|}{ Pregnancy induced hypertension (PIH) } & 050 & 051 & Pregnancy induced hypertension \\
\hline Unclassifiable nephritis & 060 & 061 & \multicolumn{2}{|c|}{\begin{tabular}{|l|} 
Other unclassifiable nephritis \\
\end{tabular}} & 060 & 061 & Unclassifiable nephritis \\
\hline Hereditary nephritis & 062 & 063 & & & & & \\
\hline Polycystic kidney diseases & 070 & 071 & \multicolumn{2}{|c|}{ Polycystic kidney diseases } & 070 & 071 & Polycystic kidney diseases \\
\hline & & & \multirow{4}{*}{$\begin{array}{l}\text { Genetic } \\
\text { disorders }\end{array}$} & Alport syndrome & 142 & 143 & \multirow{4}{*}{ Genetic disorders } \\
\hline \multirow[t]{3}{*}{ 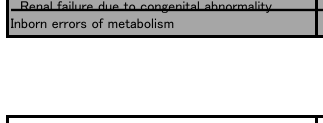 } & 740 & T41 & & Other genetic kidney diseases & 144 & 145 & \\
\hline & & & & Fabry disease & 146 & 147 & \\
\hline & & & & Kidney diseases due to other in & 148 & 149 & \\
\hline Nephrosclerosis & 080 & 081 & \multicolumn{2}{|l|}{ Nephrosclerosis } & 080 & 081 & Nephrosclerosis \\
\hline Hypertensive emergencies & 090 & 091 & \multicolumn{2}{|c|}{ Malignant hypertension or hypertensive emerge } & 090 & 091 & Malignant hypertension \\
\hline Diabetic nephropathy & 100 & 101 & \multirow{3}{*}{$\begin{array}{l}\text { Diabetic } \\
\text { nephropathy }\end{array}$} & Diabetic nephropathy & 100 & 101 & \multirow{3}{*}{ Diabetic nephropathy } \\
\hline Type 1 diabetes & 102 & 103 & & Type 1 diabetes & 102 & 103 & \\
\hline Type 2 diabetes & 104 & 105 & & Type 2 diabetes & 104 & 105 & \\
\hline SLE nephritis & 110 & 111 & Autoimmune & Lupus nephritis & 110 & 111 & Autoimmune nenhritis \\
\hline Other autoimmune disease & 112 & 113 & nephritis & \begin{tabular}{|l|} 
Other disorders due to auto-immune diseases \\
\end{tabular} & 112 & 113 & \\
\hline Amyloidal kidney & 120 & 121 & Amyloidosis & & 120 & 121 & Amyloidosis \\
\hline Gout kidney & 130 & 131 & Gout kidney & & 130 & 131 & Gout kidney \\
\hline Tuberculosis & 150 & & Urinary tract tu & uberculosis & 150 & & Urinary tract tuberculosis \\
\hline & & & Viral infection & & 260 & & Viral infection \\
\hline Urolithiasis & 160 & & Urolithiasis & & 160 & & Urolithiasis \\
\hline Kidney and urinary tract tumor & 170 & & Kidney and urin & nary tract tumor & 170 & & Kidney and urinary tract tumor \\
\hline Urinary tract obstruction & 180 & & Urinary tract of & bstruction & 180 & & Urinary tract obstruction \\
\hline Myeloma & 190 & & Paraproteinemi & ia including myeloma kidney* & 190 & & Paraproteinemia including myeloma kidney* \\
\hline & & & & Circulatory disorders & 270 & & \\
\hline & & & Acute kidney if & Thrombotic microangiopathy including TTP or HUS & 272 & & Acute kidney injury \\
\hline & & & & Other AKI & 274 & & \\
\hline & & & & Drug-induced kidney diseases & 280 & & \\
\hline & & & Extrinsic kidne & Cholesterol crystal embolism & 282 & & Extrinsic kidney disease \\
\hline & & & & Other extrinsic kidney disease & 284 & & \\
\hline Hypoplastic kidney & 200 & & $\begin{array}{l}\text { Congenital anon } \\
\text { tract(CAKUT) }\end{array}$ & naly of kidneys and urinary & 200 & & $\begin{array}{l}\text { Congenital anomaly of } \\
\text { kidneys and urinary tract }\end{array}$ \\
\hline Undetermined & 210 & & Undetermined & & 210 & & Undetermined \\
\hline Graft loss & 220 & & Graft loss & & 220 & & Graft loss \\
\hline Others & 230 & & Others & & 230 & & Others \\
\hline
\end{tabular}

*Excluding amyloidosis

of death codes were revised for better comparisons between registries in the world.

The changes to the types of primary disease codes included removal of hereditary diseases such as Alport syndrome from "Other unclassified nephritis/hereditary nephritis" and their placement under their own classification known as "Hereditary diseases," which includes the major hereditary diseases. Interstitial nephritis and nephritis 
Table 2 Kidney disease classification in JRDR report, 2017

\begin{tabular}{|c|c|c|c|}
\hline \multirow{2}{*}{ Classification in JRDR report } & \multirow{2}{*}{ Kidney disease } & \multicolumn{2}{|c|}{ Code } \\
\hline & & $\begin{array}{r}\begin{array}{c}\text { Clinical } \\
\text { diagnosis }\end{array} \\
\end{array}$ & \begin{tabular}{|c|c|}
$\begin{array}{c}\text { Pathologically } \\
\text { proven }\end{array}$ \\
\end{tabular} \\
\hline \multirow{6}{*}{ Chronic glomerulonephritis } & Chronic glomerulonephritis & 010 & 011 \\
\hline & IgA nephropathy, Henoch-Schönlein purpura & 012 & 013 \\
\hline & Other proliferative glomerulonephritis & 014 & 015 \\
\hline & Membranous nephropathy & 016 & 017 \\
\hline & Membranoproliferative glomerulonephritis & 018 & 019 \\
\hline & Focal segmental glomerulosclerosis & 240 & 241 \\
\hline Chronic pyelonephritis & Chronic pyelonephritis & 020 & 021 \\
\hline Interstitial nephritis & Interstitial nephritis & 250 & 251 \\
\hline Rapidly progressive glomerulonephriti & Rapidly progressive glomerulonephritis (ANCA-associated nephritis, anti-GBM nephritis) & 030 & 031 \\
\hline Pregnancy induced hypertension & Pregnancy induced hypertension (PIH) & 050 & 051 \\
\hline Unclassifiable nephritis & Other unclassifiable nephritis & 060 & 061 \\
\hline Polycystic kidney diseases & Polycystic kidney diseases & 070 & 071 \\
\hline \multirow{4}{*}{ Genetic disorders } & Alport syndrome & 142 & 143 \\
\hline & Other genetic kidney diseases & 144 & 145 \\
\hline & Fabry disease & 146 & 147 \\
\hline & Kidney diseases due to other inborn errors of metabolism & 148 & 149 \\
\hline Nephrosclerosis & Nephrosclerosis & 080 & 081 \\
\hline Malignant hypertension & Malignant hypertension or hypertensive emergency & 090 & 091 \\
\hline \multirow{3}{*}{ Diabetic nephropathy } & Diabetic nephropathy & 100 & 101 \\
\hline & Type 1 diabetes & 102 & 103 \\
\hline & Type 2 diabetes & 104 & 105 \\
\hline \multirow{2}{*}{ Autoimmune nephritis } & Lupus nephritis & 110 & 111 \\
\hline & Other disorders due to auto-immune diseases & 112 & 113 \\
\hline Amyloidosis & Amyloidosis & 120 & 121 \\
\hline Gout kidney & Gout kidney & 130 & 131 \\
\hline Urinary tract tuberculosis & Urinary tract tuberculosis & 150 & \\
\hline Viral infection & Viral infection & 260 & \\
\hline Urolithiasis & Urolithiasis & 160 & \\
\hline Kidney and urinary tract tumor & Kidney and urinary tract tumor & 170 & \\
\hline Urinary tract obstruction & Urinary tract obstruction & 180 & \\
\hline Paraproteinemia including myeloma $\mathrm{k}$ & Paraproteinemia including myeloma kidney* & 190 & \\
\hline \multirow{3}{*}{ Acute kidney injury } & Circulatory disorders & 270 & \\
\hline & Thrombotic microangiopathy including TTP or HUS & 272 & \\
\hline & Other AKI & 274 & \\
\hline \multirow{3}{*}{ Extrinsic kidney disease } & Drug-induced kidney diseases & 280 & \\
\hline & Cholesterol crystal embolism & 282 & \\
\hline & Other extrinsic kidney disease & 284 & \\
\hline Congenital anomaly of kidneys and ur & Congenital anomaly of kidneys and urinary tract(CAKUT) & 200 & \\
\hline Undetermined & Undetermined & 210 & \\
\hline Graft loss & Graft loss & 220 & \\
\hline Others & Others & 230 & \\
\hline
\end{tabular}

*: excluding amyloidosis 
Table 3 Codes for cause of death, comparison of the new (2017 ) and the previous (2010 2016), 2017

\begin{tabular}{|c|c|c|c|}
\hline \multirow{2}{*}{\multicolumn{2}{|c|}{ Classification of cause of death }} & \multicolumn{2}{|c|}{ Code } \\
\hline & & \multirow{2}{*}{$\begin{array}{c}\text { clinical } \\
110\end{array}$} & \multirow{2}{*}{$\begin{array}{c}\text { mefinite } \\
111\end{array}$} \\
\hline \multirow{7}{*}{$\begin{array}{c}\text { Cardiac } \\
\text { Diseases }\end{array}$} & Heart failure & & \\
\hline & Pulmonary edema (congestion) & 120 & 121 \\
\hline & Acute myocardial infarction (died & 130 & 131 \\
\hline & Ischemic heart diseases (excludir & 140 & 141 \\
\hline & Arrhythmia, conduction disorders & 150 & 151 \\
\hline & Endocarditis and Valvular disease & 160 & 161 \\
\hline & Others & 100 & 101 \\
\hline \multirow{4}{*}{$\begin{array}{c}\text { Cerebrovascula } \\
\text { r diseases }\end{array}$} & Subarachnoid hemorrhage & 210 & 211 \\
\hline & Intracerebral hemorrhage & 220 & 221 \\
\hline & Cerebral infarction & 230 & 231 \\
\hline & Others & 200 & 201 \\
\hline
\end{tabular}

\begin{tabular}{|c|c|c|c|}
\hline \multirow{2}{*}{ Sudden death } & Hyperkalemia & 910 & 911 \\
\hline & Unknown origin & 920 & 921 \\
\hline \multirow{10}{*}{$\begin{array}{l}\text { Infectious } \\
\text { diseases }\end{array}$} & Sepsis & 310 & 311 \\
\hline & Central nervous system infection & 320 & 321 \\
\hline & Pneumonia & 330 & 331 \\
\hline & Influenza & 340 & 341 \\
\hline & Urinary tract infection & 350 & 351 \\
\hline & Gastrointestinal, cholangitis, and perit & 360 & 361 \\
\hline & Fulminant (acute) viral hepatitis & 370 & 371 \\
\hline & Tuberculosis & 380 & 381 \\
\hline & Human immunodeficiency viral (HI & 390 & 391 \\
\hline & Others & 300 & 301 \\
\hline \multirow{10}{*}{ Malignancy } & Malignant neoplasm of central nervous system & 410 & 411 \\
\hline & Malignant neoplasm of respiratory system & 420 & 421 \\
\hline & Liver cancer & 430 & 431 \\
\hline & Malignant neoplasm of digestive system excluding liver cancer & 440 & 441 \\
\hline & Breast cancer & 450 & 451 \\
\hline & Genital organs malignancies & 460 & 461 \\
\hline & Malignant neoplasm of kidney & 470 & 471 \\
\hline & Endocrinological organ cancer & 480 & 481 \\
\hline & Hematological malignancies & 490 & 491 \\
\hline & Others & 400 & 401 \\
\hline \multirow{2}{*}{ Liver cirrhosis } & Viral liver cirrhosis & 510 & 511 \\
\hline & Non-viral liver cirrhosis & 520 & 521 \\
\hline
\end{tabular}

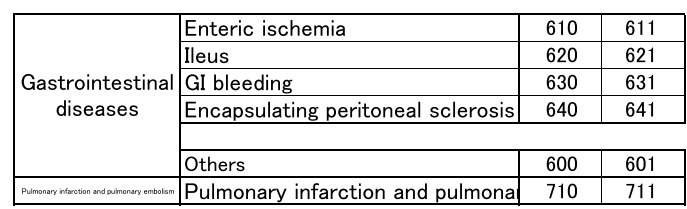

\begin{tabular}{|c|l|c|c|}
\hline \multirow{3}{*}{$\begin{array}{c}\text { Cachexia/Uremia } \\
\text { /Others }\end{array}$} & Cachexia & 810 & 811 \\
\cline { 2 - 4 } & Uremia & 820 & 821 \\
\cline { 2 - 4 } & Dementia & 830 & 831 \\
\cline { 2 - 4 } & Others & 800 & 801 \\
\hline
\end{tabular}

\begin{tabular}{|l|l|c|c|}
\hline \multirow{3}{*}{$\begin{array}{c}\text { Suicide, refuse, } \\
\text { accident, and } \\
\text { withdrawal }\end{array}$} & Suicide & 010 & \\
\cline { 2 - 4 } & Dreatment refuse & 020 & \\
\cline { 2 - 5 } & Disaster or accident & 030 & 031 \\
\hline Others & Others & 080 & 081 \\
\hline Undetermined & Undetermined & 090 & \\
\hline
\end{tabular}

\begin{tabular}{|c|c|c|c|}
\hline \multicolumn{2}{|c|}{ Category of cause of death } & \multirow{2}{*}{\begin{tabular}{|c|} 
Code \\
110 \\
\end{tabular}} & \multirow{2}{*}{$\begin{array}{c}\begin{array}{c}\text { Classification in } \\
\text { JRDR report }\end{array} \\
\text { Heart failure }\end{array}$} \\
\hline \multirow{9}{*}{$\begin{array}{l}\text { Cardiac } \\
\text { Diseases }\end{array}$} & Heart failure & & \\
\hline & Pulmonary edema (congestion) & 120 & \\
\hline & Acute myocardial infarction (died wit & 130 & \multirow{2}{*}{$\begin{array}{l}\text { Myocardial } \\
\text { infarction }\end{array}$} \\
\hline & Ischemic heart diseases (excluding & 140 & \\
\hline & Arrhythmia, conduction disorders & 150 & \multirow{5}{*}{ Heart failure } \\
\hline & Valvular heart diseases* & 162 & \\
\hline & Epicarditis & 170 & \\
\hline & Cardiomyopathy & 180 & \\
\hline & Others & 100 & \\
\hline \multirow{4}{*}{$\begin{array}{c}\text { Cerebrovascular } \\
\text { diseases }\end{array}$} & Subarachnoid hemorrhage & 210 & \multirow{4}{*}{$\begin{array}{c}\text { Cerebrovascula } \\
\text { diseases }\end{array}$} \\
\hline & Cerabral hemorrhage & 220 & \\
\hline & Cerebral infarction & 230 & \\
\hline & Others & 200 & \\
\hline \multirow{2}{*}{$\begin{array}{l}\text { Vascular } \\
\text { diseases }\end{array}$} & Aortic aneurysm (including dissectio & 260 & \multirow{2}{*}{ Others } \\
\hline & Others & 250 & \\
\hline \multirow{2}{*}{ Sudden death } & Hyperkalemia & 910 & Hyperkalemia/ \\
\hline & Unknown origin & 920 & sudden death \\
\hline & Sepsis & 310 & \\
\hline & Central nervous system infection & 320 & \\
\hline & Pneumonia & 330 & \\
\hline & Influenza & 340 & \\
\hline Infectious & Urinary tract infection & 350 & Infectious \\
\hline diseases & Gastrointestinal, cholangitis, and peritor & 360 & diseases \\
\hline & Infective endocarditis & 164 & \\
\hline & Tuberculosis & 380 & \\
\hline & Human immunodeficiency viral (HIV) & 390 & \\
\hline & Others & 300 & \\
\hline & Malignant neoplasm of central nervous system & 410 & \\
\hline & Malignant neoplasm of respiratory system & 420 & \\
\hline & Hepatocellular carcinoma & 430 & \\
\hline & Gastric cancer & 442 & \\
\hline & Colon and rectal cancer & 444 & \\
\hline & Pancreatic cancer & 446 & \\
\hline & Gall bladder or bile ductal cancer & 448 & \\
\hline Malignancy & Other GI tract malignancies & 440 & Malignancy \\
\hline & Breast cancer & 450 & \\
\hline & Genital organs malignancies & 460 & \\
\hline & Renal cell carcinoma & 472 & \\
\hline & Urinary tract malignancies other that & 474 & \\
\hline & Endocrinological organ cancer & 480 & \\
\hline & Hematological malignancies & 490 & \\
\hline & Others & 400 & \\
\hline & Viral liver cirrhosis & 510 & rhosis \\
\hline & Non-viral liver cirrhosis & 520 & hosis \\
\hline Liver, bile duct, & Fulminant hepatitis & 370 & Others \\
\hline and pancreatic & Acute liver failure other than fulmin & 530 & Others \\
\hline & Pancreatitis & 540 & Others \\
\hline & Others & 500 & Others \\
\hline & Enteric ischemia & 610 & \\
\hline & Ileus & 620 & lleus \\
\hline Gastrointestinal & GI bleeding & 630 & GI bleeding \\
\hline diseases & Encapsulating peritoneal sclerosis & 640 & Ileus \\
\hline & GI tract perforation & 650 & Others \\
\hline & Others & 600 & Others \\
\hline Lung and & Pulmonary infarction and pulmonary & 710 & \\
\hline respiratory & Chronic obstructive pulmonary disea & 720 & $\begin{array}{l}\text { Pulmonary } \\
\text { disease }\end{array}$ \\
\hline diseases & Others excluding pneumonia & 700 & \\
\hline Hematological & Bone marrow failure & 760 & Hematological \\
\hline & Others & 750 & \\
\hline & Cachexia & 810 & \\
\hline & Uremia & 820 & \\
\hline $\begin{array}{l}\text { Cacnexla/uremI } \\
\text { a/senility }\end{array}$ & 'Senility (no other causes than older & 840 & Cachexia/uremi \\
\hline & Dementia & 830 & \\
\hline & Other cachexia or uremia & 800 & \\
\hline Endocrinological & Endocrinological or metabolic diseas & 850 & Others \\
\hline & Suicide & 010 & Suicide/refu \\
\hline Suicide / refuse & Treatment refuse & 020 & SuIcide/refuse \\
\hline / accident / & Disaster or accident & 030 & Disaster or accident \\
\hline withdrawal & Poisoning & 040 & Others \\
\hline & Withdrawal & 050 & Others \\
\hline Others & Others & 080 & Others \\
\hline Undetermined & Undetermined & 090 & Undetermined \\
\hline
\end{tabular}


Table 4 Cause of death classification in JRDR report, 2017

\begin{tabular}{|c|c|c|c|c|c|}
\hline \multirow{2}{*}{ Cause of death classification } & \multirow{2}{*}{ Cause of death $\left(2017^{\sim}\right)$} & \multirow[b]{2}{*}{ Code } & \multirow{2}{*}{ Cause of death $\left(2010^{2} 2016\right)$} & \multicolumn{2}{|c|}{ Code } \\
\hline & & & & $\begin{array}{l}\text { Without } \\
\text { clinical } \\
\text { definite }\end{array}$ & $\begin{array}{l}\text { With } \\
\text { clinical } \\
\text { definite }\end{array}$ \\
\hline \multirow[t]{7}{*}{ Heart failure } & Heart failure & 110 & Heart failure & 110 & 111 \\
\hline & Pulmonary edema (congestion) & 120 & Pulmonary edema (congestion) & 120 & 121 \\
\hline & Arrhythmia, conduction disorders & 150 & Arrhythmia, conduction disorders & 150 & 151 \\
\hline & Valvular heart diseases** & 162 & Endocarditis and Valvular disease & 160 & 161 \\
\hline & Epicarditis & 170 & & & \\
\hline & Cardiomyopathy & 180 & & & \\
\hline & Others & 100 & Others & 100 & 101 \\
\hline \multirow{4}{*}{ Cerebrovascular diseases } & Subarachnoid hemorrhage & 210 & Subarachnoid hemorrhage & 210 & 211 \\
\hline & Cerabral hemorrhage & 220 & Intracerebral hemorrhage & 220 & 221 \\
\hline & Cerebral infarction & 230 & Cerebral infarction & 230 & 231 \\
\hline & Others & 200 & Others & 200 & 201 \\
\hline \multirow{11}{*}{ Infectious diseases } & Sepsis & 310 & Sepsis & 310 & 311 \\
\hline & Central nervous system infection & 320 & Central nervous system infection & 320 & 321 \\
\hline & Pneumonia & 330 & Pneumonia & 330 & 331 \\
\hline & Influenza & 340 & Influenza & 340 & 341 \\
\hline & Urinary tract infection & 350 & Urinary tract infection & 350 & 351 \\
\hline & Gastrointestinal, cholangitis, and peritonitis & 360 & Gastrointestinal, cholangitis, and peritonitis & 360 & 361 \\
\hline & & & Fulminant (acute) viral hepatitis & 370 & 371 \\
\hline & Tuberculosis & 380 & Tuberculosis & 380 & 381 \\
\hline & Human immunodeficiency viral (HIV) infection & 390 & Human immunodeficiency viral (HIV) infection & 390 & 391 \\
\hline & Infective endocarditis & 164 & & & \\
\hline & Others & 300 & Others & 300 & 301 \\
\hline GI bleeding & GI bleeding & 630 & GI bleeding & 630 & 631 \\
\hline \multirow{15}{*}{ Malignancy } & Malignant neoplasm of central nervous system & 410 & Malignant neoplasm of central nervous & 410 & 411 \\
\hline & Malignant neoplasm of respiratory system & 420 & Malignant neoplasm of respiratory syste & 420 & 421 \\
\hline & Hepatocellular carcinoma & 430 & Liver cancer & 430 & 431 \\
\hline & Gastric cancer & 442 & & & \\
\hline & Colon and rectal cancer & 444 & Malignant neoplasm of digestive & & \\
\hline & Pancreatic cancer & 446 & system & 440 & 441 \\
\hline & Gall bladder or bile ductal cancer & 448 & excluding liver cancer & & \\
\hline & Other GI tract malignancies & 440 & & & \\
\hline & Breast cancer & 450 & Breast cancer & 450 & 451 \\
\hline & Genital organs malignancies & 460 & Genital organs malignancies & 460 & 461 \\
\hline & Renal cell carcinoma & 472 & Malignant neoplasm of kidney & 470 & 471 \\
\hline & Urinary tract malignancies other than RCC & 474 & & & \\
\hline & Endocrinological organ cancer & 480 & Endocrinological organ cancer & 480 & 481 \\
\hline & Hematological malignancies & 490 & Hematological malignancies & 490 & 491 \\
\hline & Others & 400 & Others & 400 & 401 \\
\hline Cachexia/uremia/senility & Cachexia & 810 & Cachexia & 810 & 811 \\
\hline & Uremia & 820 & Uremia & 820 & 821 \\
\hline & Senility (no other causes than older age) & 840 & & & \\
\hline & Dementia & 830 & Dementia & 830 & 831 \\
\hline & Other cachexia or uremia & 800 & Other cachexia or uremia & 800 & 801 \\
\hline Myocardial infarction & Acute myocardial infarction (died within 30 & 130 & Acute myocardial infarction (died within & 130 & 131 \\
\hline & Ischemic heart diseases (excluding AMI) & 140 & Ischemic heart diseases (excluding AM & 140 & 141 \\
\hline Hyperkalemia/Sudden death & Hyperkalemia & 910 & Hyperkalemia & 910 & 911 \\
\hline & Unknown origin & 920 & Unknown origin & 920 & 921 \\
\hline Liver cirrhosis & Viral liver cirrhosis & 510 & Viral liver cirrhosis & 510 & 511 \\
\hline & Non-viral liver cirrhosis & 520 & Non-viral liver cirrhosis & 520 & 521 \\
\hline Suicide/Refuse & Suicide & 010 & Suicide & 010 & \\
\hline & Treatment refuse & 020 & Treatment refuse & 020 & \\
\hline Ileus & Enteric ischemia & 610 & Enteric ischemia & 610 & 611 \\
\hline & Ileus & 620 & Ileus & 620 & 621 \\
\hline & Encapsulating peritoneal sclerosis & 640 & Encapsulating peritoneal sclerosis & 640 & 641 \\
\hline Hematological disease & Bone marrow failure & 760 & & & \\
\hline & Others & 750 & & & \\
\hline Pulmonary diseases & Pulmonary infarction and pulmonary embolism & 710 & Pulmonary infarction and pulmonary embolism & 710 & 711 \\
\hline & Chronic obstructive pulmonary disease or chron & 720 & & & \\
\hline & Others excluding pneumonia & 700 & & & \\
\hline Disaster or accident & Disaster or accident & 030 & Disaster or accident & 030 & 031 \\
\hline Others & Aortic aneurysm (including dissection) & 260 & & & \\
\hline & Other vascular diseases & 250 & & & \\
\hline & Fulminant hepatitis & 370 & & & \\
\hline & Acute liver failure other than fulminant hepatitis & 530 & & & \\
\hline & Pancreatitis & 540 & & & \\
\hline & Other liver biliary tract diseases & 500 & & & \\
\hline & GI tract perforation & 650 & & & \\
\hline & Other gastrointestinal diseases & 600 & Other gastrointestinal diseases & 600 & 601 \\
\hline & Endocrinological or metabolic diseases & 850 & & & \\
\hline & Poisoning & 040 & & & \\
\hline & Withdrawal & 050 & & & \\
\hline & Others & 080 & Others & 080 & 081 \\
\hline Undetermined & Undetermined & 090 & Undetermined & 090 & \\
\hline
\end{tabular}

**Infective endocarditis, formerly included, is classified under infectious diseases. 
Table 5 Summary of chronic dialysis therapy in Japan, 2017

\begin{tabular}{|c|c|c|c|c|c|c|c|}
\hline \multicolumn{2}{|c|}{ Number of surveyed faciliteis } & \multicolumn{2}{|c|}{4,413 facilities } & \multicolumn{4}{|c|}{ (increas of 17 facilities, $0.4 \%$ increase) } \\
\hline \multicolumn{2}{|c|}{ Number of responded facilities } & \multicolumn{2}{|c|}{ 4,360 facilities } & \multicolumn{4}{|c|}{ (increas of 24 facilities, $0.6 \%$ increase) } \\
\hline \multirow{3}{*}{ Capacity } & Number of bedside consoles & \multicolumn{2}{|c|}{137,248 units } & \multicolumn{4}{|c|}{ (increase of 2,037 units, $1.5 \%$ increase) } \\
\hline & Capacity for simultaneous HD treatments & \multicolumn{2}{|c|}{135,636 treatments } & \multicolumn{4}{|c|}{ (increase of 2,136 patients, $1.6 \%$ increase) } \\
\hline & Maximum capacity & \multicolumn{2}{|c|}{ 450,838patients } & \multicolumn{4}{|c|}{ (increase of 6,646 patientns, $1.5 \%$ increase } \\
\hline \multirow{2}{*}{\multicolumn{2}{|c|}{ Prevalent dialysis patients }} & \multicolumn{2}{|c|}{334,505 patients } & \multicolumn{4}{|c|}{ (increase of 4,896 patients, $1.5 \%$ increase) } \\
\hline & & \multicolumn{2}{|c|}{ Outpatients } & \multicolumn{2}{|c|}{ Inpatients } & \multicolumn{2}{|c|}{ Total } \\
\hline \multirow{5}{*}{ Hemodialysis } & \multirow{5}{*}{$\begin{array}{l}\text { Hemodialysis (HD) } \\
\text { Hemodiafiltration (HDF) } \\
\text { Hemofiltration (HF) } \\
\text { Blood adsorption dialysis } \\
\text { Home hemodialysis }\end{array}$} & 203,024 & $(66.7)$ & 25,065 & $(83.0)$ & 228,089 & $(68.2)$ \\
\hline & & 90,537 & $(29.8)$ & 4,603 & $(15.2)$ & 95,140 & $(28.4)$ \\
\hline & & 21 & $(0.0)$ & 19 & $(0.1)$ & 40 & $(0.0)$ \\
\hline & & 1,407 & $(0.5)$ & 55 & $(0.2)$ & 1,462 & $(0.4)$ \\
\hline & & 683 & $(0.2)$ & 1 & $(0.0)$ & 684 & $(0.2)$ \\
\hline \multirow{6}{*}{$\begin{array}{l}\text { Peritoneal } \\
\text { dialysis }\end{array}$} & \multirow{5}{*}{$\begin{array}{l}\text { PD only } \\
P D+\text { HD 1/week } \\
P D+\text { HD 2/week } \\
P D+\text { HD 3/week } \\
\text { PD + HD other frequencies }\end{array}$} & 6,946 & (2.3) & 379 & (1.3) & 7,325 & (2.2) \\
\hline & & 1,475 & $(0.5)$ & 30 & $(0.1)$ & 1,505 & $(0.4)$ \\
\hline & & 152 & $(0.0)$ & 3 & $(0.0)$ & 155 & $(0.0)$ \\
\hline & & 25 & $(0.0)$ & 12 & $(0.0)$ & 37 & $(0.0)$ \\
\hline & & 47 & $(0.0)$ & 21 & $(0.1)$ & 68 & $(0.0)$ \\
\hline & Subtotal & 8,645 & $(2.8)$ & 445 & $(1.5)$ & 9,090 & $(2.7)$ \\
\hline \multicolumn{2}{|l|}{ Total } & 304,317 & $(100.0)$ & 30,188 & $(100.0)$ & 334,505 & $\overline{(100.0)}$ \\
\hline \multicolumn{2}{|c|}{$\begin{array}{l}\text { Per million of general population } \\
\text { Patients count in the night shift }\end{array}$} & \multicolumn{2}{|c|}{$\begin{array}{l}2,640.0 \text { patients } \\
31,916 \text { patients }\end{array}$} & \multicolumn{4}{|c|}{ (increase of 43.3 patients) } \\
\hline \multicolumn{2}{|c|}{ Incident dialysis patients } & \multicolumn{2}{|c|}{40,959 patients } & \multicolumn{4}{|c|}{ (increase of 1,615 patients, $4.1 \%$ increase) } \\
\hline \multicolumn{2}{|c|}{ Incident hemodialysis patients (including HDF) } & \multicolumn{6}{|c|}{38,842 patients } \\
\hline \multicolumn{2}{|c|}{ Incident peritoneal dialysis patients } & $2,117 \mathrm{pa}$ & ents & & & & \\
\hline Deceased pa & atients & $32,532 \mathrm{pa}$ & ents & (increase & f 742 pati & nts, $2.3 \%$ & rease) \\
\hline
\end{tabular}

PD + HD patients: Patients treated by the combination of PD and HD, HDF, hemoadsorption, or hemofiltration (excluding those who underwent only peritoneal lavage)

* The above data were obtained from the facility survey.

PD + HD patients: patients treated by the combination of PD and HD, HDF, HAD, or HF (excluding those who underwent only peritoneal lavage)

*The above data were obtained from the facility survey

associated with autoimmune diseases were also reclassified. New codes were created for handling delayed acute kidney injury and renal failure caused by exogenous kidney injury (Table 1). These modifications were used to create primary disease categories for use in data aggregation for surveys conducted as of 2017 with consideration paid to continuity with surveys conducted in 2016 and earlier (Table 2).

Changes made to the cause of death code classifications include splitting the heart disease category of "Endocarditis and valvular disease" into "Valvular disease," "Pericarditis," "Cardiomyopathy," and "Other heart diseases" as well as classifying endocarditis as an infectious disease under the heading "infectious endocarditis." In addition, a cause of death code for "vascular diseases," which includes aortic aneurysm, was newly created. The sub-categories within the malignant tumor code were increased and it was made easier to identify the incidence rates for malignant tumors in each organ. Finally, new cause of death codes was created for hepatobiliary and pancreatic diseases, lung and respiratory diseases, and hematologic diseases (Table 3). These modifications were used to create the cause of death classifications for use in data aggregation for surveys conducted as of 2017 with consideration paid to continuity with surveys conducted in 2016 and earlier (Table 4).

\section{Questionnaire recovery status}

The 2017 survey targeted 4413 facilities throughout Japan. Completed facility-survey questionnaires were recovered from 4360 facilities (98.8\%). This represents a $0.6 \%$ increase (+24 facilities) over the previous year. Patientsurvey questionnaires were recovered from 4188 facilities 


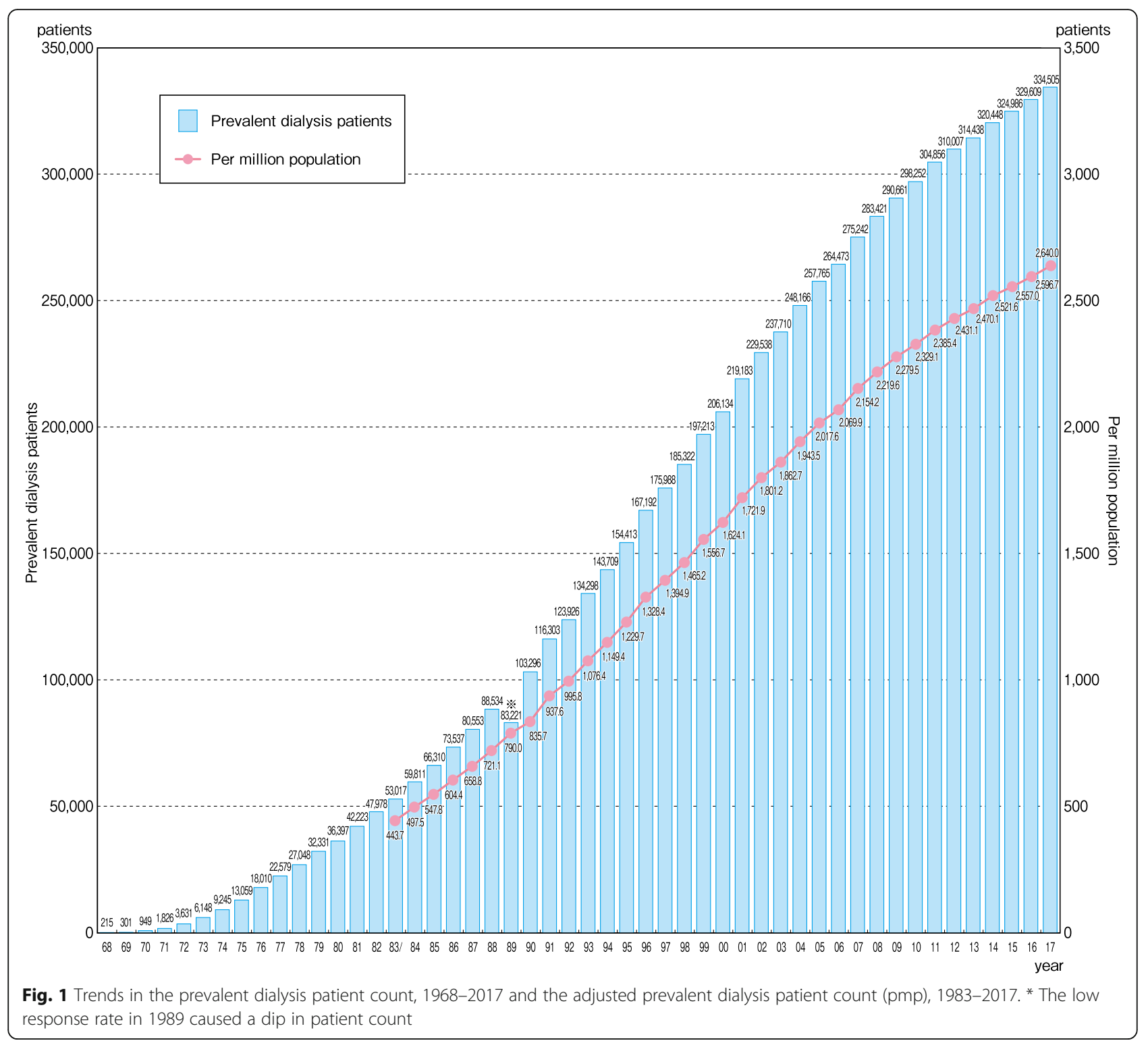

(94.9\%). The paper-based patient survey was discontinued in 2015, but this had no effect on the recovery rate.

\section{Part II. 2017 JSDT survey report: results and discussion}

\section{Chapter 1: basic demographics}

\section{Facility dynamics}

The 2017 JRDR survey targeted 4413 facilities throughout Japan and recovered completed questionnaires from 4360 facilities. Although the number of facilities that returned facility-survey questionnaires fell temporarily in 2015 (nine fewer facilities as compared to the previous year: $-0.2 \%$ ), the number increased in $2016(+15$ facilities, $+0.3 \%)$ and increased again in 2017 (+24 facilities, $+0.6 \%)$. Although the number of returned patient-survey questionnaires fell temporarily as a result of the discontinuation of the paper- based survey in association with improved anonymization methods that were implemented in 2015, as of the 2017 survey the recovery rate for the facility-survey questionnaire was $98.8 \%$ (4360 facilities) and the recovery rate for the patient-survey questionnaire was $94.9 \%$ of the total (4188 facilities; Table 5). The results for the targeted facilities show that there were 137,248 dialysis consoles, simultaneous dialysis capacity of 135,636 , and that the maximum dialysis treatment capacity of 450,838 , which represent a $1.5 \%, 1.6 \%$, and $1.5 \%$ increase over the previous year, respectively. The number of dialysis consoles is increasing annually (Additional file 1: Table S1).

\section{Patient dynamics}

Based on the facility-survey questionnaire results, the total number of patients undergoing chronic dialysis treatment 
at the end of 2017 was 334,505 . This number indicates the prevalence of regular chronic kidney disease (CKD) patients undergoing dialysis treatment. Although the number of patients undergoing dialysis is increasing annually, the rate of increase has slowed in recent years. In 2017, there was an increase of 4896 patients as compared to the previous year (Fig. 1, Additional file 1: Table S1). A future prediction of the number of dialysis patients that was conducted by Nakai et al. [5] in 2012 indicated that the number was expected to decline after reaching a peak of approximately 349,000 in 2021 . The number of dialysis patients per 1 million population indicates the prevalence rate (Fig. 1, Additional file 1: Table S1). The prevalence rate has been on an increasing trend in recent years, with the figure at 2640 per 1 million population. This indicates that one in every 378.1 Japanese are dialysis patients. According to the United States Renal Data System (USRDS), the prevalence of dialysis patients is highest in the world in Taiwan, with Japan following in second place [6].

The number of new dialysis patients indicates the incidence of CKD patients who are undergoing dialysis treatment. This figure is increasing annually. Although almost plateauing since 2008, incidence has been increasing since then, with the incidence in 2017 at 40,959 (Fig. 2, Additional file 2: Table S2). Of these, $94.8 \%$ were $\mathrm{HD}(\mathrm{F})$ and $5.2 \%$ were $\mathrm{PD}$ (Table 5). The number of deceased patients has been on an annually increasing trend. Although it almost plateaued between 2011 and 2014, since 2015, the figure has once again been on increasing, with 32,532 deceased patients in 2017 (Fig. 2, Additional file 2: Table S2). In general, the number of patients for any given fiscal year is calculated by adding the number of new patients to the number of patients from the previous fiscal year and then subtracting the number of deceased patients. However, as this figure may not include the

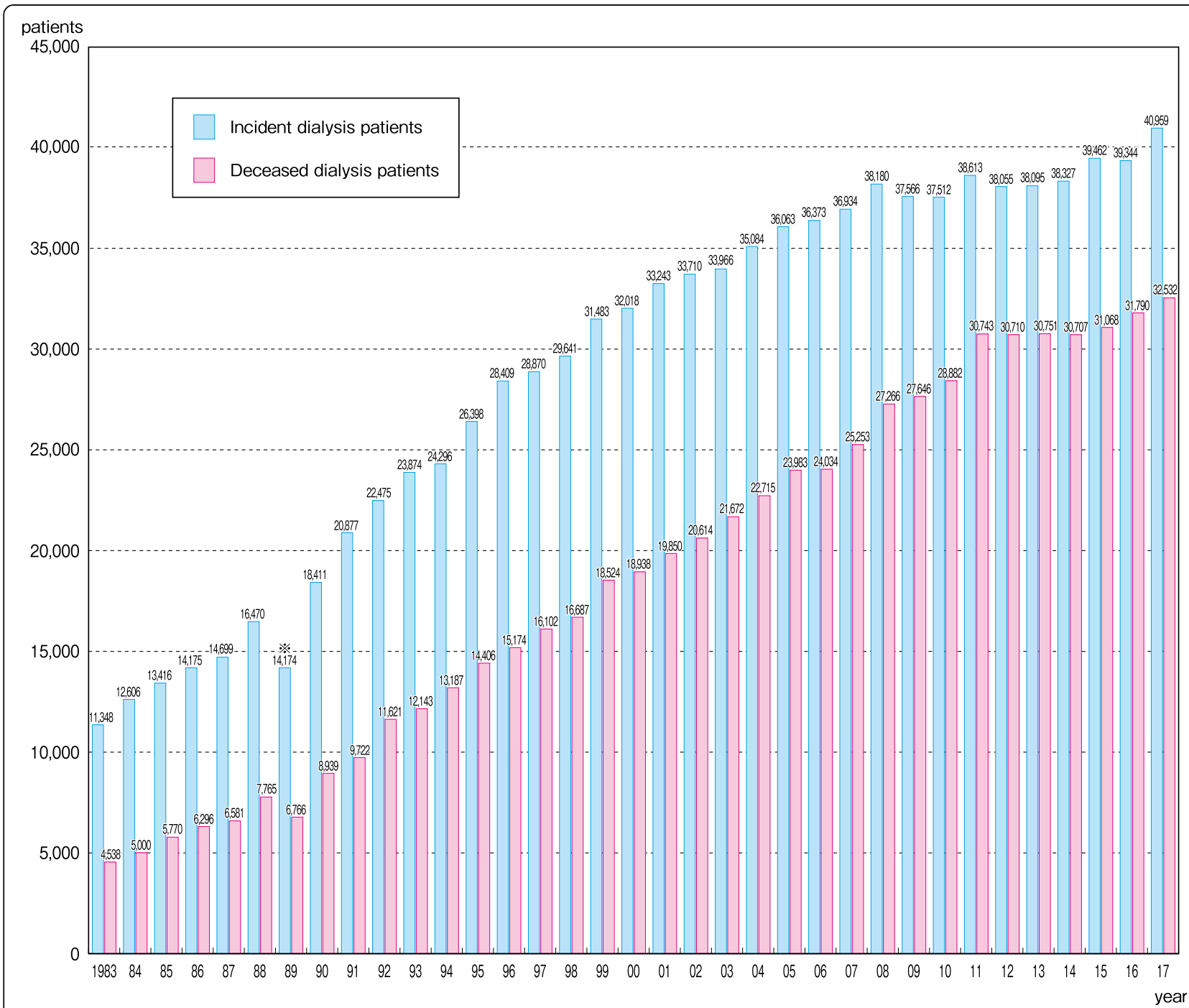

Fig. 2 Trends in the incident and deceased dialysis patient counts, 1983-2017 
number of patients who discontinued dialysis due to transplantation and because the number of new patients may be overestimated while the number of deceased patients may be underestimated, the number of patients thus calculated may not be consistent with the actual number of patients.

The numbers of dialysis patients by prefecture are shown in Table 6 . The prefectural totals shown in the

Table 6 Prevalent dialysis patient count, by modality \& prefecture, 2017

\begin{tabular}{|c|c|c|c|c|c|c|c|c|c|c|c|c|c|c|}
\hline \multirow{2}{*}{ Prefecture } & \multirow{2}{*}{$\begin{array}{c}\text { Number of } \\
\text { surveyed } \\
\text { faciliteis }\end{array}$} & \multirow{2}{*}{\begin{tabular}{|l|}
$\begin{array}{l}\text { Number of } \\
\text { responded } \\
\text { facilities }\end{array}$ \\
\end{tabular}} & \multicolumn{5}{|c|}{ Hemodialysis } & \multicolumn{5}{|c|}{ Peritoneal dialysis } & \multirow{2}{*}{ Total } & \multirow{2}{*}{\begin{tabular}{|l} 
Per million \\
of general \\
population
\end{tabular}} \\
\hline & & & Hemodialysis & Hemodiafitratic & Hemofittration & - & Homen hemodialaysis & PD only & $\mathrm{PD}+\mathrm{HD}$ 1/week & PD + HD 2/week & $\mathrm{PD}+\mathrm{HD}$ 3/week & & & \\
\hline Hokkaido & 261 & 260 & 9,659 & 5,533 & 0 & 81 & 10 & 309 & 67 & 4 & 3 & 9 & 15,675 & $2,946.4$ \\
\hline Aomori & 40 & 40 & 1,718 & 1,791 & 0 & 8 & 3 & 53 & 9 & 2 & 0 & 0 & 3,584 & $2,804.4$ \\
\hline Iwate & 44 & 44 & 2,701 & 365 & 0 & 11 & 0 & 81 & 12 & 1 & 0 & 0 & 3,171 & $2,526.7$ \\
\hline Miyagi & 63 & 63 & 4,152 & 1,477 & 0 & 14 & 1 & 64 & 10 & 2 & 0 & 2 & 5,722 & $2,463.2$ \\
\hline Akita & 43 & 42 & 1,483 & 618 & 0 & 4 & 2 & 50 & 3 & 0 & 0 & 0 & 2,160 & $2,168.7$ \\
\hline Yamagata & 36 & 35 & 1,753 & 834 & 0 & 4 & 12 & 44 & 7 & 4 & 1 & 0 & 2,659 & $2,412.9$ \\
\hline Fukushima & 69 & 67 & 3,343 & 1,498 & 0 & 20 & 0 & 80 & 29 & 12 & 1 & 0 & 4,983 & $2,647.7$ \\
\hline Ibaraki & 86 & 84 & 6,090 & 1,823 & 0 & 36 & 13 & 71 & 16 & 0 & 2 & 1 & 8,052 & $2,784.2$ \\
\hline Tochigi & 77 & 75 & 4,589 & 1,307 & 0 & 14 & 3 & 96 & 18 & 1 & 0 & 0 & 6,028 & $3,080.2$ \\
\hline Gunma & 63 & 63 & 4,380 & 1,514 & 0 & 0 & 11 & 46 & 16 & 0 & 2 & 0 & 5,969 & $3,045.4$ \\
\hline Saitama & 189 & 187 & 11,649 & 6,126 & 1 & 66 & 77 & 243 & 76 & 11 & 2 & 1 & 18,252 & $2,496.9$ \\
\hline Chiba & 154 & 153 & 10,220 & 4,855 & 1 & 32 & 9 & 193 & 63 & 4 & 3 & 2 & 15,382 & $2,462.7$ \\
\hline Tokyo & 435 & 429 & 19,926 & 10,800 & 5 & 161 & 97 & 896 & 247 & 12 & 3 & 7 & 32,154 & $2,342.9$ \\
\hline Kanagawa & 262 & 259 & 15,194 & 5,251 & 16 & 71 & 27 & 519 & 75 & 1 & 1 & 1 & 21,156 & $2,309.9$ \\
\hline Niigata & 54 & 54 & 4,285 & 762 & 1 & 22 & 1 & 150 & 20 & 1 & 1 & 1 & 5,244 & $2,313.2$ \\
\hline Toyama & 42 & 42 & 1,924 & 501 & 0 & 11 & 3 & 94 & 14 & 1 & 1 & 0 & 2,549 & $2,413.8$ \\
\hline Ishikawa & 41 & 41 & 2,108 & 525 & 0 & 24 & 5 & 68 & 7 & 0 & 0 & 0 & 2,737 & $2,386.2$ \\
\hline Fukui & 25 & 24 & 1,030 & 658 & 0 & 5 & 3 & 81 & 20 & 6 & 0 & 1 & 1,804 & $2,315.8$ \\
\hline Yamanashi & 33 & 33 & 1,436 & 839 & 0 & 5 & 2 & 27 & 11 & 0 & 0 & 2 & 2,322 & 2,821.4 \\
\hline Nagano & 73 & 73 & 3,273 & 1,919 & 0 & 14 & 16 & 83 & 16 & 5 & 0 & 0 & 5,326 & $2,565.5$ \\
\hline Gifu & 72 & 72 & 3,868 & 1,031 & 0 & 30 & 26 & 67 & 14 & 1 & 0 & 0 & 5,037 & $2,508.5$ \\
\hline Shizuoka & 124 & 124 & 6,562 & 4,435 & 1 & 43 & 20 & 97 & 22 & 6 & 0 & 1 & 11,187 & $3,044.1$ \\
\hline Aichi & 194 & 193 & 13,598 & 3,870 & 0 & 93 & 47 & 588 & 100 & 3 & 0 & 0 & 18,299 & $2,431.8$ \\
\hline Mie & 53 & 50 & 3,266 & 679 & 0 & 21 & 6 & 56 & 12 & 0 & 0 & 0 & 4,040 & 2,244.4 \\
\hline Shiga & 40 & 39 & 2,186 & 857 & 0 & 27 & 31 & 109 & 27 & 0 & 0 & 0 & 3,237 & $2,290.9$ \\
\hline Kyoto & 79 & 78 & 4,637 & 1,646 & 0 & 85 & 11 & 159 & 62 & 6 & 1 & 3 & 6,610 & $2,543.3$ \\
\hline Osaka & 321 & 316 & 16,428 & 6,648 & 2 & 158 & 43 & 427 & 83 & 7 & 2 & 3 & 23,801 & $2,697.6$ \\
\hline Hyogo & 200 & 197 & 8,944 & 4,716 & 9 & 97 & 73 & 154 & 33 & 9 & 1 & 0 & 14,036 & $2,550.6$ \\
\hline Nara & 45 & 45 & 2,093 & 1,089 & 1 & 33 & 8 & 109 & 31 & 1 & 2 & 0 & 3,367 & $2,497.8$ \\
\hline Wakayama & 48 & 47 & 2,429 & 473 & 0 & 15 & 28 & 49 & 7 & 0 & 0 & 0 & 3,001 & $3,175.7$ \\
\hline Tottori & 26 & 26 & 892 & 583 & 0 & 3 & 2 & 47 & 10 & 0 & 0 & 0 & 1,537 & $2,720.4$ \\
\hline Shimane & 29 & 28 & 780 & 799 & 0 & 1 & 1 & 58 & 9 & 1 & 0 & 0 & 1,649 & $2,407.3$ \\
\hline Okayama & 67 & 67 & 3,205 & 1,670 & 0 & 27 & 5 & 172 & 9 & 4 & 0 & 0 & 5,092 & $2,670.2$ \\
\hline Hiroshima & 99 & 98 & 4,588 & 2,709 & 1 & 31 & 31 & 212 & 55 & 40 & 5 & 1 & 7,673 & $2,712.3$ \\
\hline Yamaguchi & 61 & 57 & 1,874 & 1,510 & 0 & 6 & 1 & 73 & 25 & 1 & 1 & 1 & 3,492 & $2,524.9$ \\
\hline Tokushima & 38 & 38 & 1,491 & 1,107 & 0 & 7 & 5 & 128 & 36 & 2 & 0 & 1 & 2,777 & $3,737.6$ \\
\hline Kagawa & 48 & 48 & 1,484 & 997 & 0 & 8 & 9 & 150 & 39 & 0 & 1 & 1 & 2,689 & $2,780.8$ \\
\hline Ehime & 53 & 53 & 2,206 & 1,623 & 0 & 9 & 0 & 91 & 27 & 0 & 1 & 16 & 3,973 & $2,912.8$ \\
\hline Kochi & 39 & 38 & 1,288 & 1,084 & 0 & 7 & 0 & 15 & 3 & 0 & 1 & 0 & 2,398 & $3,358.5$ \\
\hline Fukuoka & 198 & 195 & 11,634 & 2,481 & 1 & 47 & 16 & 666 & 45 & 2 & 1 & 1 & 14,894 & $2,916.4$ \\
\hline Saga & 36 & 36 & 1,812 & 624 & 0 & 6 & 1 & 10 & 5 & 0 & 0 & 0 & 2,458 & $2,983.0$ \\
\hline Nagasaki & 63 & 62 & 3,181 & 681 & 0 & 18 & 15 & 108 & 8 & 0 & 0 & 1 & 4,012 & $2,963.1$ \\
\hline Kumamoto & 89 & 89 & 5,239 & 1,065 & 0 & 34 & 3 & 132 & 24 & 0 & 1 & 1 & 6,499 & $3,682.2$ \\
\hline Oita & 70 & 67 & 3,144 & 593 & 0 & 9 & 4 & 117 & 29 & 4 & 0 & 0 & 3,900 & $3,385.4$ \\
\hline Miyazaki & 65 & 64 & 3,139 & 665 & 0 & 6 & 0 & 63 & 1 & 0 & 0 & 3 & 3,877 & $3,560.1$ \\
\hline Kagoshima & 94 & 94 & 4,334 & 933 & 1 & 24 & 1 & 116 & 29 & 1 & 0 & 5 & 5,444 & $3,348.1$ \\
\hline Okinawa & 72 & 71 & 2,874 & 1,576 & 0 & 14 & 2 & 104 & 24 & 0 & 0 & 3 & 4,597 & $3,185.7$ \\
\hline Total & 4,413 & 4,360 & $\begin{array}{r}228,089 \\
(68.2)\end{array}$ & $\begin{array}{r}95,140 \\
(28.4)\end{array}$ & $\begin{array}{r}40 \\
(0.0)\end{array}$ & $\begin{array}{r}1,462 \\
(0.4)\end{array}$ & $\begin{array}{r}684 \\
(0.2)\end{array}$ & $\begin{array}{r}7,325 \\
(2.2)\end{array}$ & $\begin{array}{r}1,505 \\
(0.4)\end{array}$ & $\begin{array}{r}155 \\
(0.0)\end{array}$ & $\begin{array}{r}37 \\
(0.0)\end{array}$ & $\begin{array}{r}68 \\
(0.0)\end{array}$ & $\begin{array}{c}334,505 \\
(100.0)\end{array}$ & $2,640.0$ \\
\hline
\end{tabular}

* The above data were obtained from the facility survey.

** The numbers of dialysis patients were adjusted as per million population ( $\mathrm{pmp}$ ) by the annual governement report. reference(7)

*The above data were obtained from the facility survey

**The numbers of dialysis patients were adjusted as per million population (pmp) by the annual government report. Reference (7) 


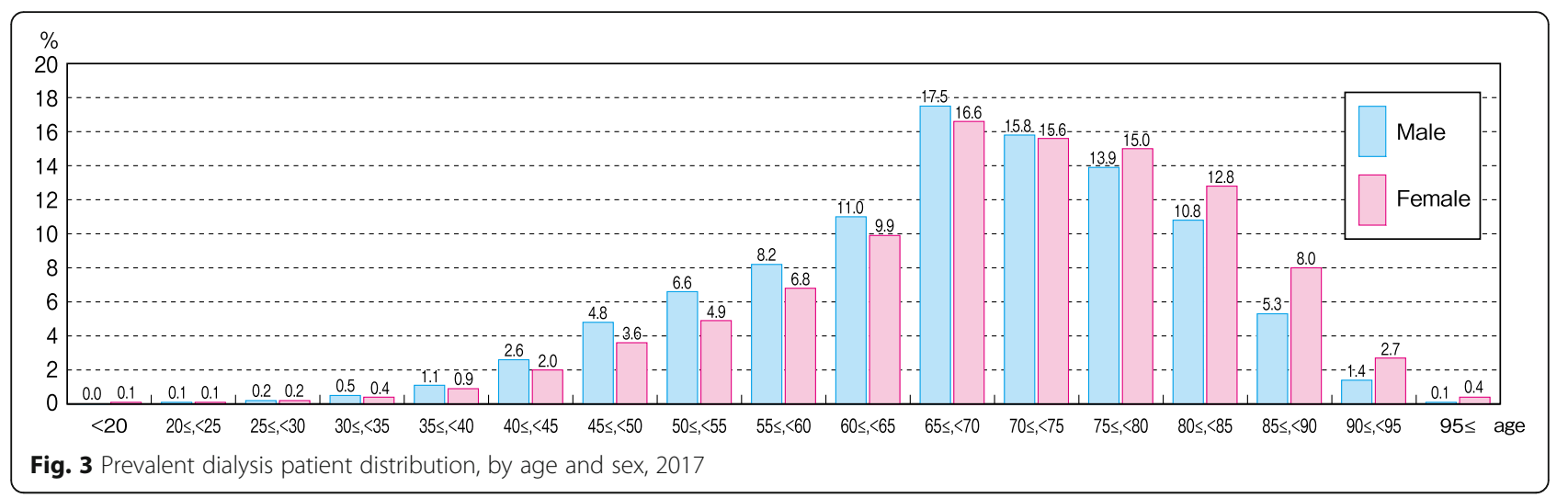

table were calculated based on the location of the facility where the patients undergo treatment and not using the locations where the patients reside. Thus, care must be exercised as, strictly speaking, these figures do not reflect patient dynamics by prefecture. The prevalence rate (number of dialysis patients per 1 million population) differs considerably from one region to another. An extremely large number of complex confounding factors are involved, and as a result great caution must be exercised when comparing prefectures.

\section{Dialysis modality dynamics}

Hemodialysis (HD) accounted for $68.2 \%$ of all dialysis modalities during 2017, followed by hemodiafiltration (HDF) at $28.4 \%$, hemofiltration (HF) at $0.01 \%$, hemadsorption dialysis (HAD) at $0.4 \%$, home hemodialysis (HHD) at $0.2 \%$, and peritoneal dialysis (PD) at $2.7 \%$ (Table 5). On-line HDF showed rapid increases after the 2012 revision to the medical reimbursement system, and in 2017 the number of HDF patients had increased overall to 95,140 . The number of patients who underwent PD was 9090, which was a slight increase over the 9021 from the previous year. Of those, 19.4\% underwent on the combination with $\mathrm{HD}(\mathrm{F})$. The number of HHD patients was 684, which represented an, albeit slight, increase. The total percentage of patients undergoing home dialysis, which is calculated by adding the number undergoing PD and HHD, was $2.9 \%$. This figure is the lowest for this type of dialysis in the developed world [6]. Although there were regional differences in the treatment type data by prefecture, these are affected by various regional factors (Table 6).

The numbers of patients undergoing nighttime dialysis were estimated to have been between 41,000 and 42,000 until the 2014 survey. This number was 33,370 in 2015, 32,431 in 2016, and 31,916 in 2017, indicating a downward trend (Table 5). This is likely to have been affected by the addition of the phrase "Dialysis during the time period recognized by the insurance system (start at 5 PM or later or finish after 9 PM or later)" to the definition of nighttime dialysis patients in the 2015 survey.

\section{Chapter 2: prevalent dialysis patient dynamics at the end of 2017}

\section{Clinical background}

Of the total 321,516 patients from the patient survey, 208,870 were male and 112,646 were female (Fig. 3, Additional file 3: Table S3). The mean age was 68.43 years, indicating a gradual annual increase (Fig. 4, Additional file 4: Table S4). The age group of 65 to 69 had the highest percentage both in males and females. The number of patients aged 65 years and under showing decline from 2012 onward. Expressed another way, this indicates that the increases in the

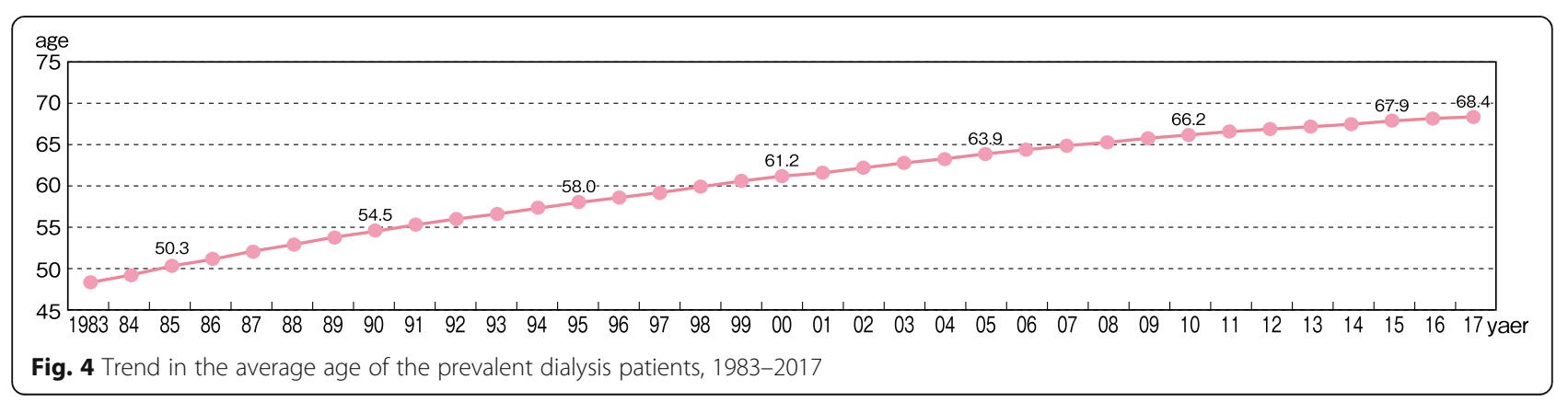




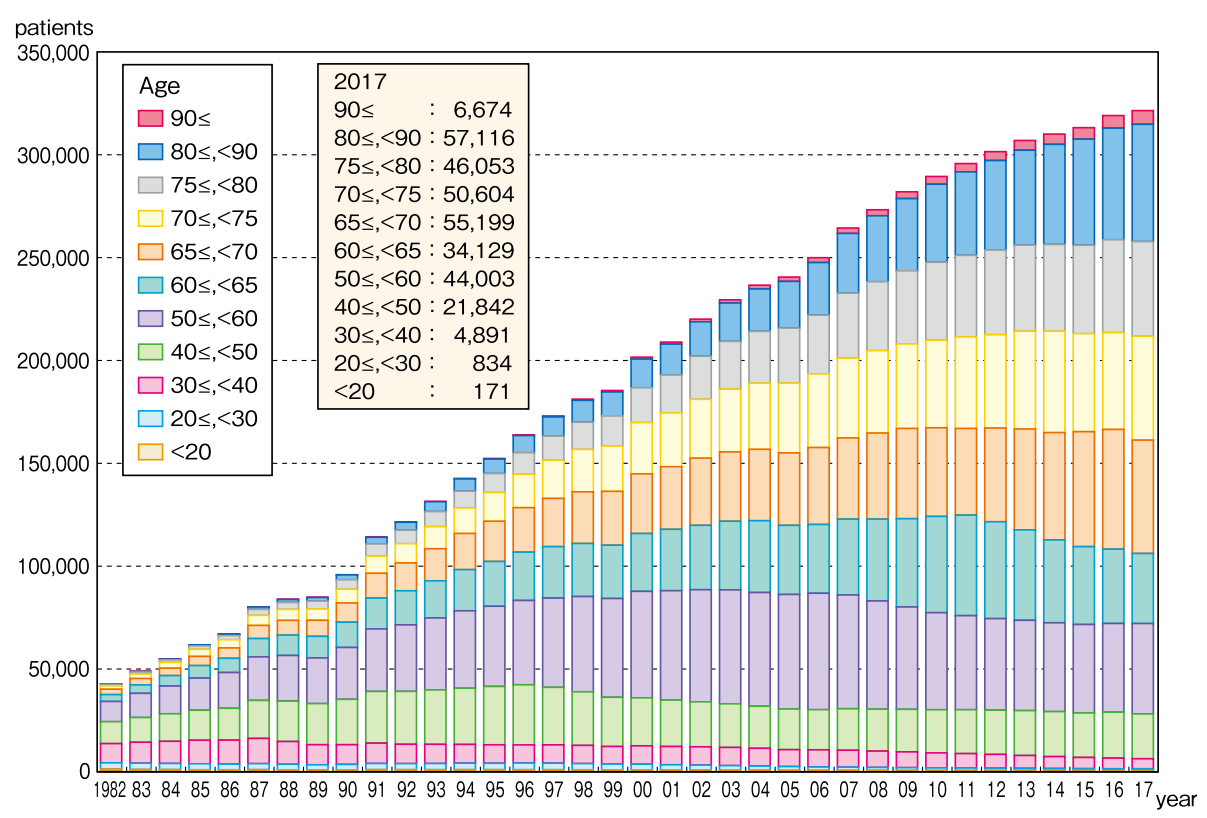

Fig. 5 Prevalent dialysis patient count, by age, 1982-2017

number of regular dialysis patients in Japan are due to increases in the number of patients aged 65 years and older (Fig. 5, Additional file 5: Table S5).

The mean dialysis vintage for chronic dialysis patients at the end of 2017 was 6.82 years for males and 8.30 years for females (7.34 years overall). Comparison of dialysis vintage by vintage groups indicates that $47.4 \%$ had a dialysis vintage of under 5 years, $8.3 \%$ had a vintage of 20 or more years, $2.2 \%$ had a vintage of 30 or more years, and $0.3 \%$ had a vintage of 40 or more years (Fig. 6 , Additional file 6: Table S6). The longest vintage was 49 years 4 months. The numbers of patients with longer vintages are on the increase, with the number of patients on dialysis for ten or more years at $27.8 \%$. Patients with a dialysis vintage of 20 or more years, which accounted for less than $1 \%$ at the end of 1992 , had reached $8.3 \%$ at the end of 2017 (Fig. 7, Additional file 7: Table S7).

The most common primary disease among chronic dialysis patients at the end of 2017 was diabetic nephropathy at $39.0 \%$, followed by chronic glomerulonephritis at $27.8 \%$, and nephrosclerosis at $10.3 \%$ (Fig. 8, Additional file 8: Table S8). The percentage of diabetic nephropathy has continuously increased and that it replaced chronic glomerulonephritis as the most common primary disease in 2011. Subsequent to 2011, the percentage of diabetic nephropathy patients has continuously increased, although the rate of increase has slowed in recent years. The percentage of chronic glomerulonephritis patients has steadily declined, while the percentages of nephrosclerosis and "undetermined" patients have continuously increased (Fig. 9, Additional file 9: Table S9). It should be considered to evaluate overtime changes of the primary diseases that the primary disease code was revised as of the 2017 survey.

\section{Causes of death}

Although 32,532 deaths were reported in the 2017 facilitysurvey questionnaire, the number of patients whose cause of death by sex was recorded in the patient-survey questionnaire was 31,139. Causes of death, in descending order, were heart failure, infectious disease, malignancy, and cerebrovascular disease $(24.0 \%, 21.1 \%, 9.0 \%$, and $6.0 \%$ respectively). The "Other" category accounted for $10.4 \%$ overall. The percentage of patients in the "cardiovascular death" category, which includes heart failure, cerebrovascular disease, and myocardial infarction, was 33.8\% (Fig. 10, Additional file 10: Table S10).

Heart failure was the most common cause of death from 1983 onward and that it accounted for approximately $25 \%$ of all deaths from 1995 onward. Death due to infectious disease, on the other hand, has been on increasing since 1993. Cerebrovascular disease has been gradually decreasing at a stable rate since 1994. Deaths from myocardial infarction have been gradually decreasing since the peak of $8.4 \%$ recorded in 1997. Malignancy deaths were at their lowest in 1987 at 5.8\% and, although they increased slightly since that time, they have remained in the $9.0 \%$ range since 2004 . The percentage of cardiovascular deaths mentioned above have consistently declined since reaching $54.8 \%$ in 1988, and in 2017 they were at $33.8 \%$ (Fig. 11, Additional file 11: Table 


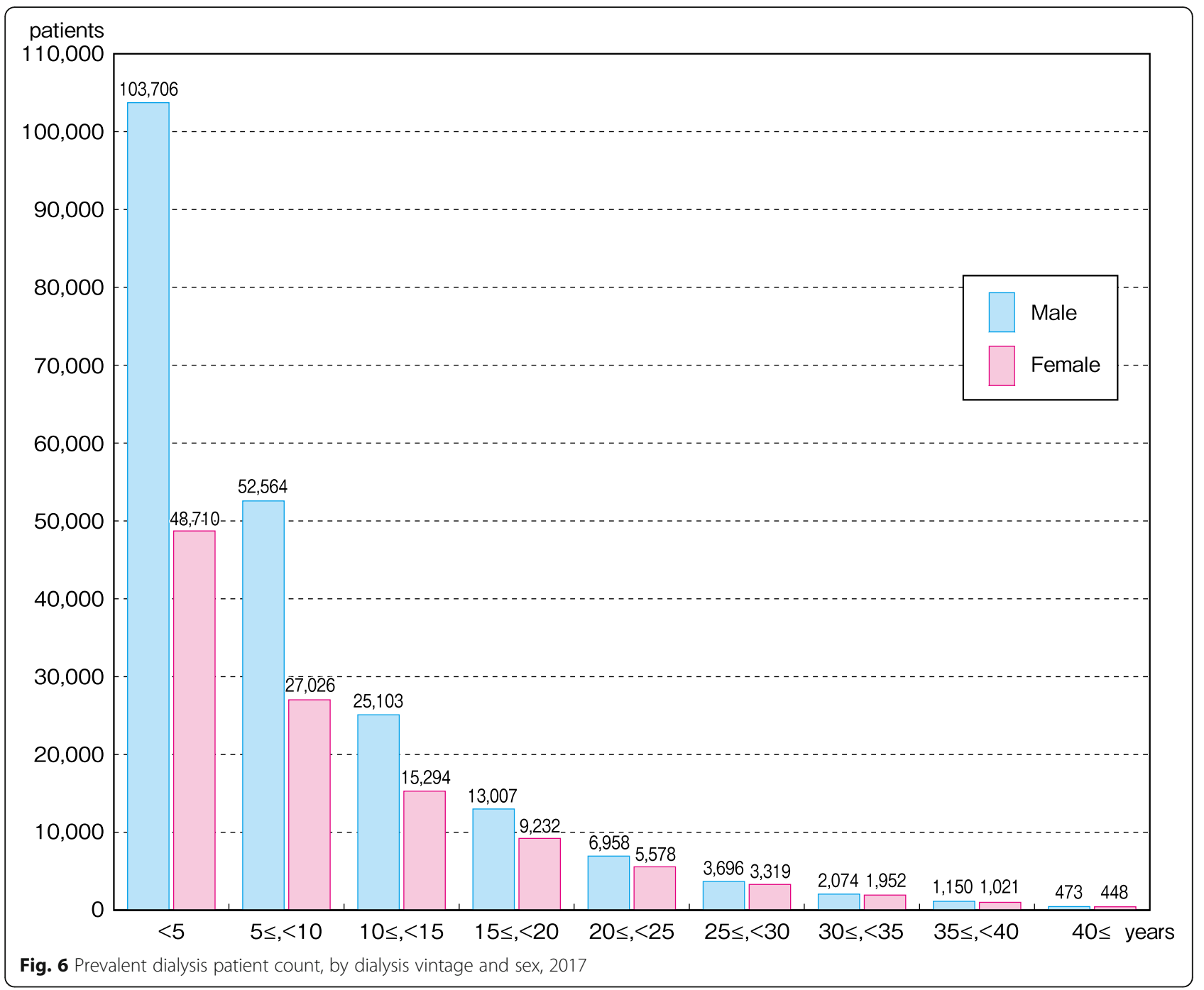

S11). Caution is required when viewing these statistics, however, as the cause of death codes were revised three times at the end of 2003, 2010, and 2017 [7].

\section{Crude death rate}

The annual crude death rate is calculated using the patient dynamics in the facility survey.

Crude death rate $=$ \{no. of deaths $/$ (no. of deaths, previous yr. + no. of patients, target yr.) $\div 2\} \times 100$ (\%)

The crude death rate indicates that the lowest rate was 7.9\% in 1989 (a year in which the questionnaire recovery rate was low), but generally fluctuates between 9 and $10 \%$. At the end of 2017, it was $9.8 \%$ (Fig. 12, Additional file 12: Table S12).

\section{Chapter 3: incident dialysis patient dynamics in 2017 Clinical background}

Of the total 38,786 incident patients whose age and sex data were included in the patient survey, 26,677 were male and 12,109 were female (Fig. 13, Additional file 13: Table S13). The mean age of the incident patients was 69.68 years (males: 68.90 years, females: 71.41 years). The mean age has been increasing annually (Fig. 14, Additional file 14: Table S14). Observation of the incident patient age data in 5-year age groups indicates that the higher age groups account for the largest percentages of patients, with males at 75-79 years and females at 80-84 years.

The most common primary disease among incident patients in 2017 was diabetic nephropathy at $42.5 \%$, followed by chronic glomerulonephritis at $16.3 \%$, nephrosclerosis at $14.7 \%$, and "undetermined" at $13.2 \%$ (Fig. 15, Additional file 15: Table S15). In 1998, diabetic nephropathy supplanted chronic glomerulonephritis as the most common primary disease among incident patients; the distribution of diabetic nephropathy has increased consistently ever since, but it has remained nearly the same in the past few years. In contrast, the percentages 


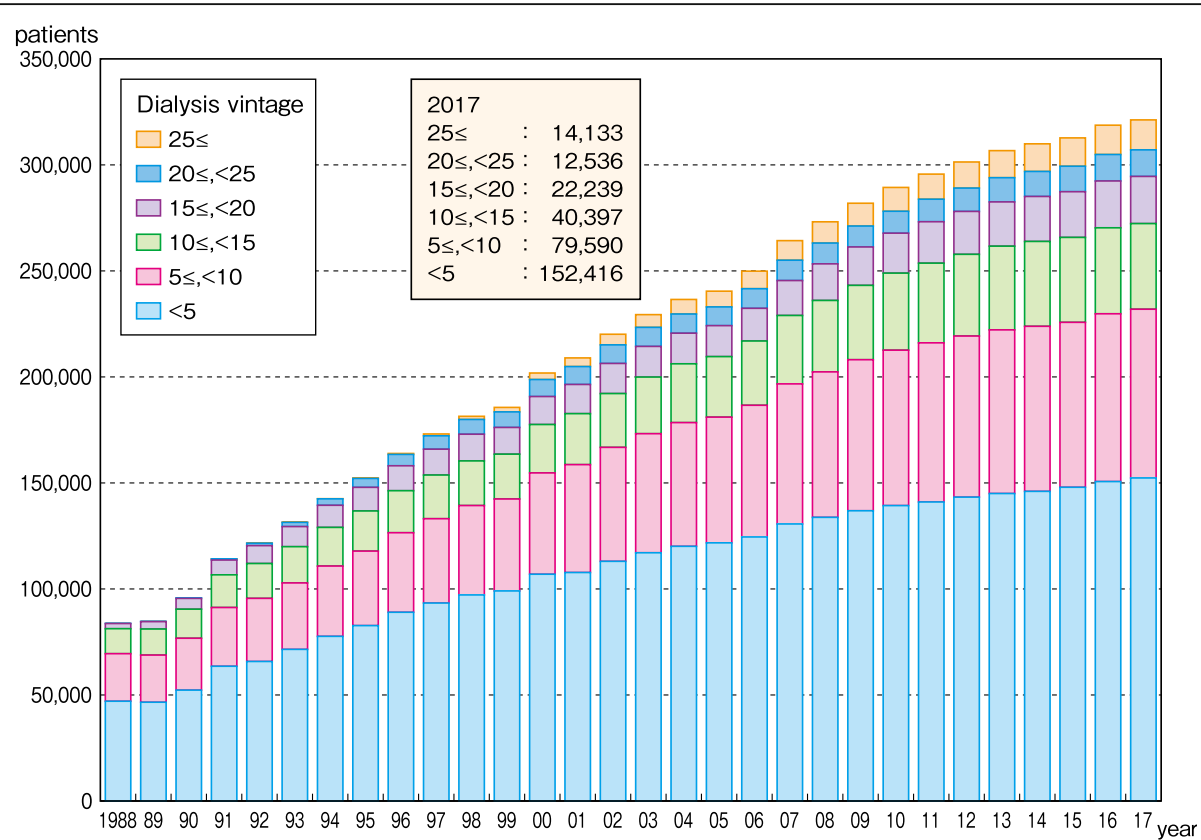

Fig. 7 Prevalent dialysis patient count, by dialysis vintage, 1988-2017

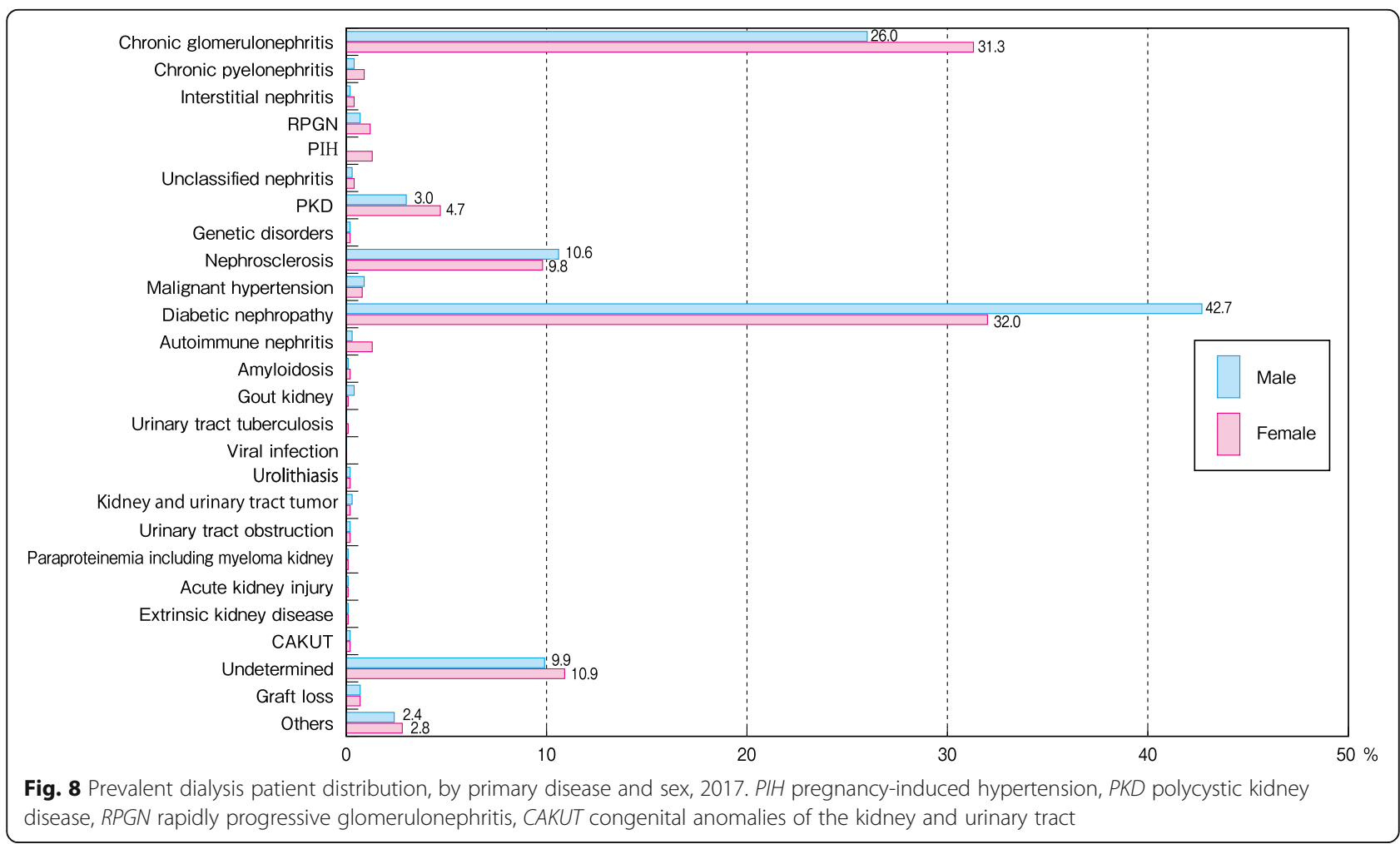




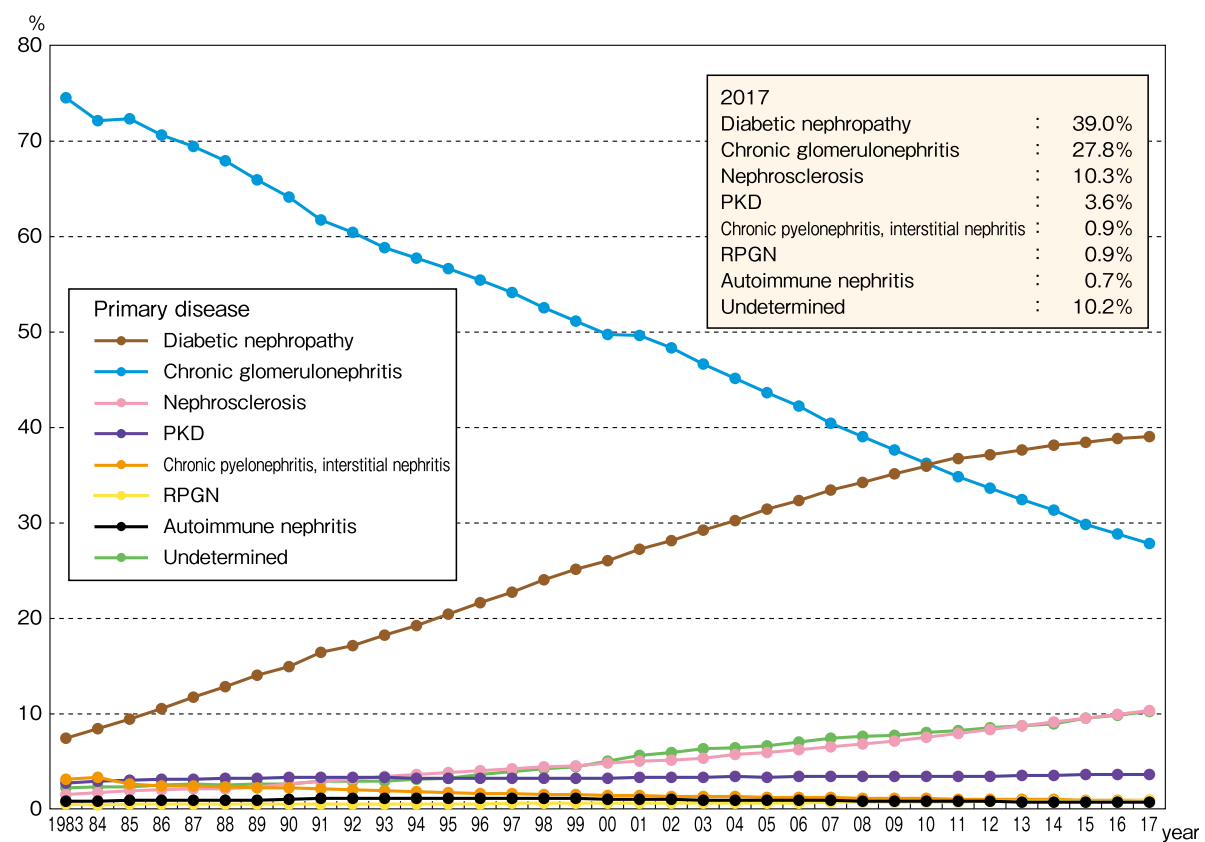

Fig. 9 Trends in major primary diseases of the prevalent dialysis patients, 1983-2017. PKD polycystic kidney disease, RPGN rapidly progressive glomerulonephritis

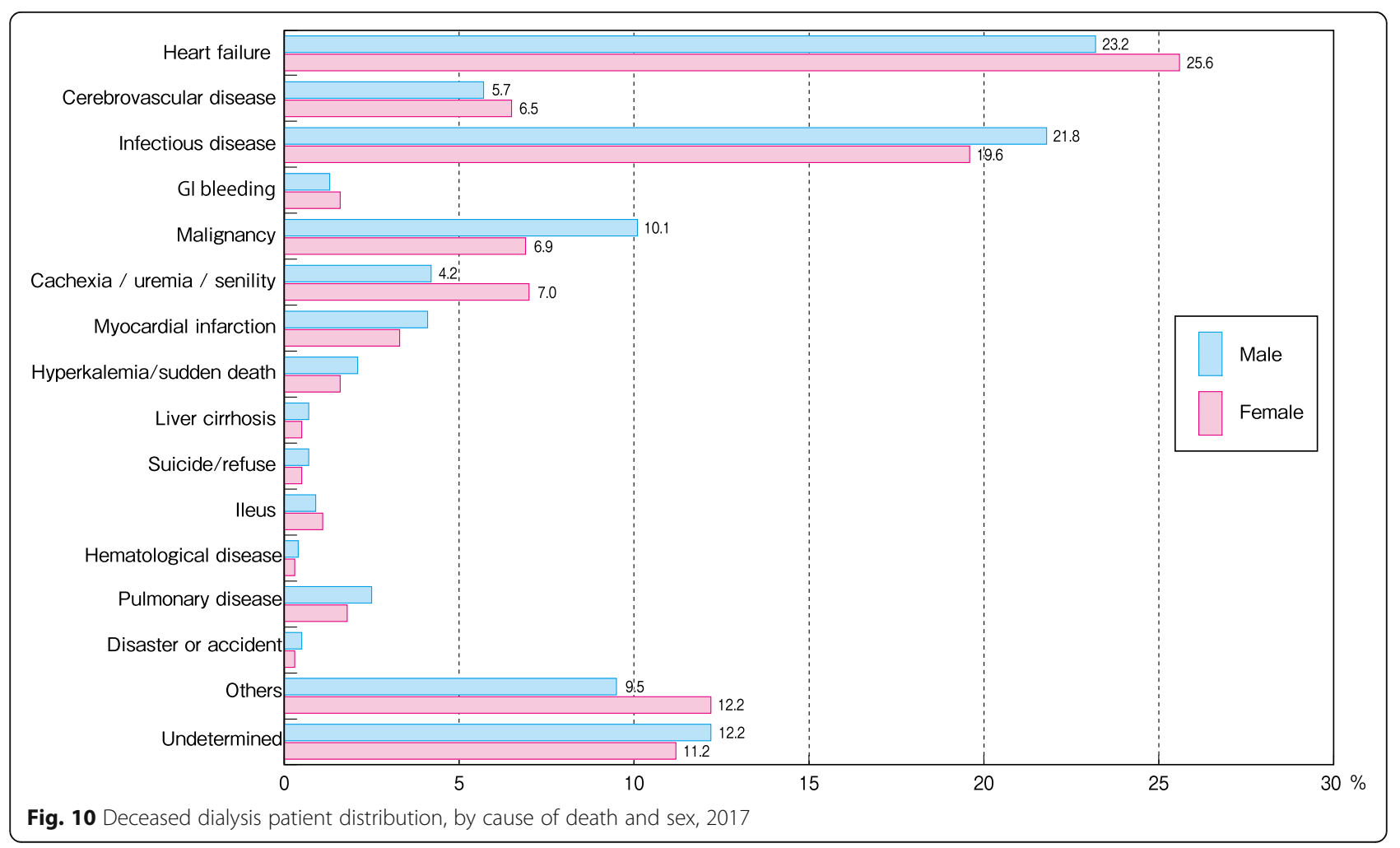




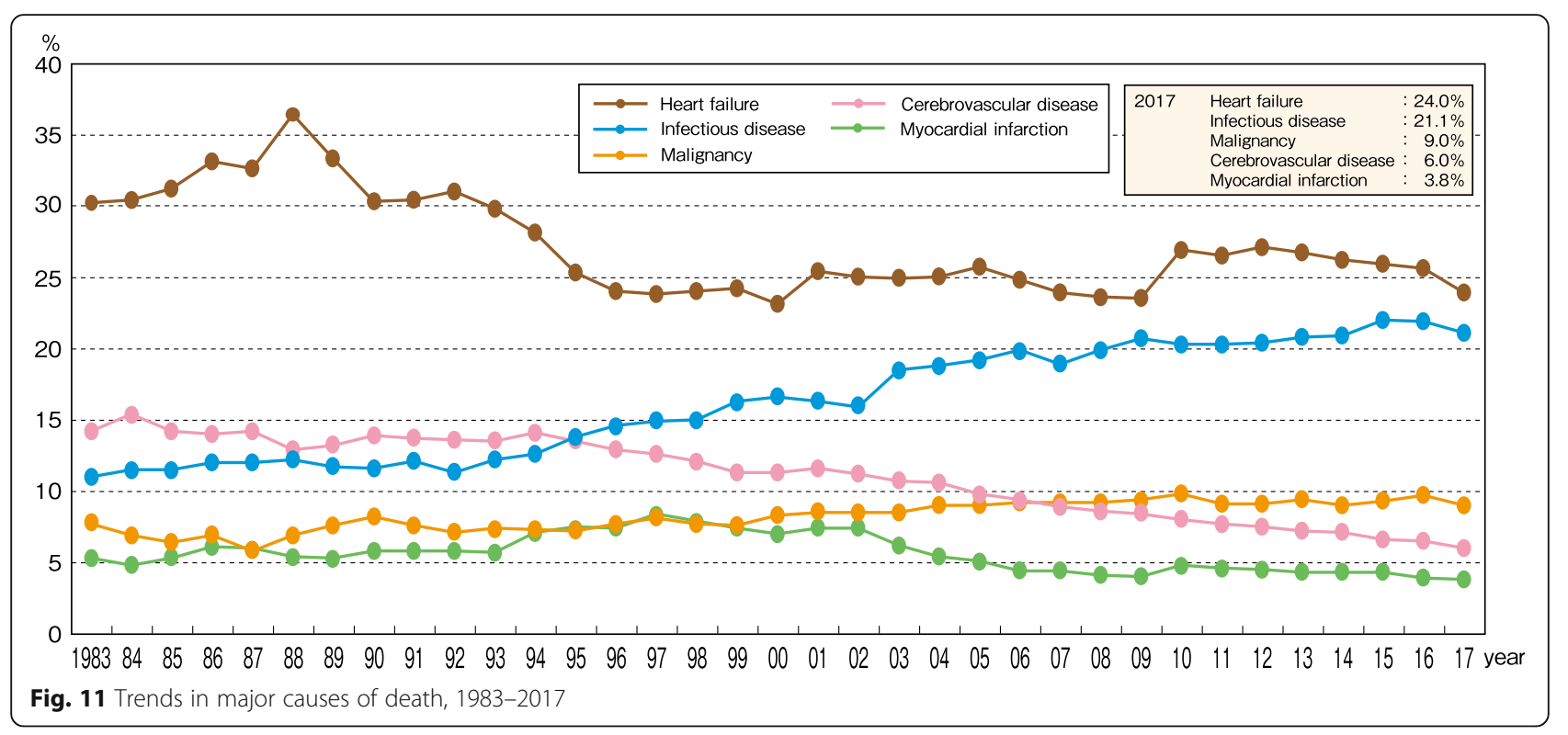

of patients with nephrosclerosis and "undetermined" have increased annually (Fig. 16, Additional file 16: Table S16).

\section{Causes of death}

In 2017 incident patients, the most common cause of death was infectious disease at $25.9 \%$, followed by heart failure at $20.8 \%$, malignancy at $10.8 \%$, cerebrovascular disease at $4.5 \%$, and myocardial infarction at $3.2 \%$. The total percentage of cardiovascular deaths was $28.5 \%$ (Fig. 17, Additional file 17: Table S17). Heart failure continued to decrease in 2016 and 2017. The changes in the causes of death within the dialysis incident year show that in the 1990s, heart failure was the most common, while infectious disease gradually increased until surpassing heart failure in 2006 when infectious disease became the most common cause of death. Deaths due to malignancy have been increasing and the percentage surpassed $10 \%$ in 2006. Deaths due to cerebrovascular disease have been gradually decreasing (Fig. 18, Additional file 18: Table S18).

\section{Chapter 4: management for dialysis fluid quality Background and subjects}

As of the 2006 survey, JSDT has been surveying the bacteriological dialysis fluid quality and the management status of this quality. Based on the results thus obtained, the bacteriological standard for dialysis fluid was revised in 2008 [8] and a chemical contamination standard was newly added in 2016 [9].

These standards assess the bacteriological standard of the dialysis fluid using the endotoxin (ET) level and the total viable microbial count (TVC). Both are assessed at least once per month. Each dialysis console is tested at a rate of at least one console per month and all consoles are tested at a rate of at least once per year. The minimum standard required for use in dialysis treatment is designated as the "Standard dialysis fluid." Specifically, this indicates an ET level of under $0.05 \mathrm{EU} / \mathrm{mL}$ and TVC of under $100 \mathrm{cfu} / \mathrm{mL}$. Ultrapure dialysis fluid (UPD) is defined as having an ET level of under 0.001 $\mathrm{EU} / \mathrm{mL}$ and a TVC of under $0.1 \mathrm{cfu} / \mathrm{mL}$. UPD is

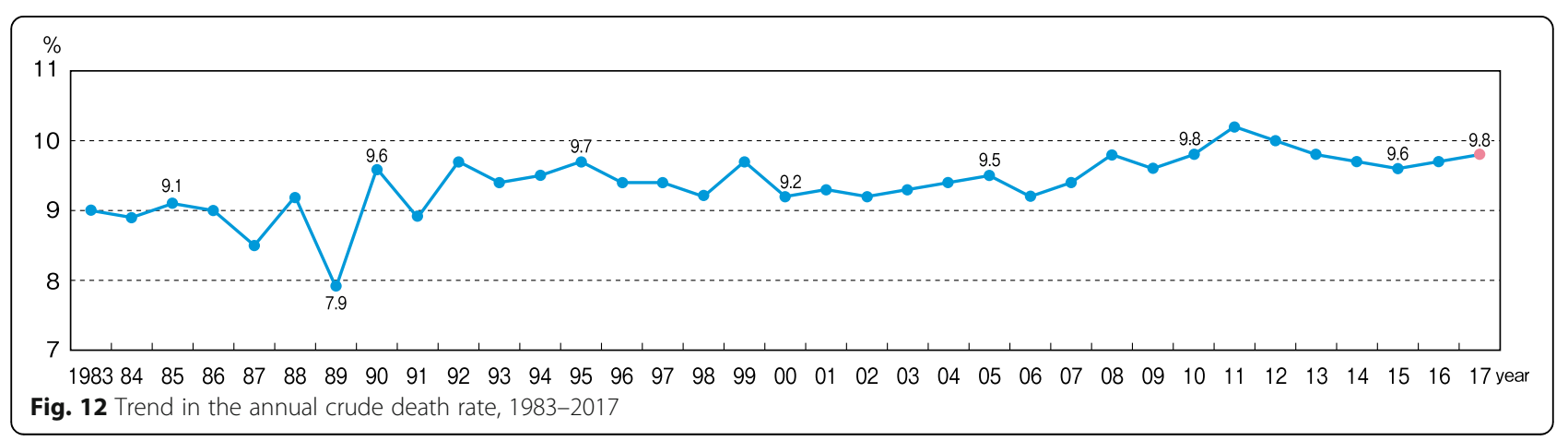




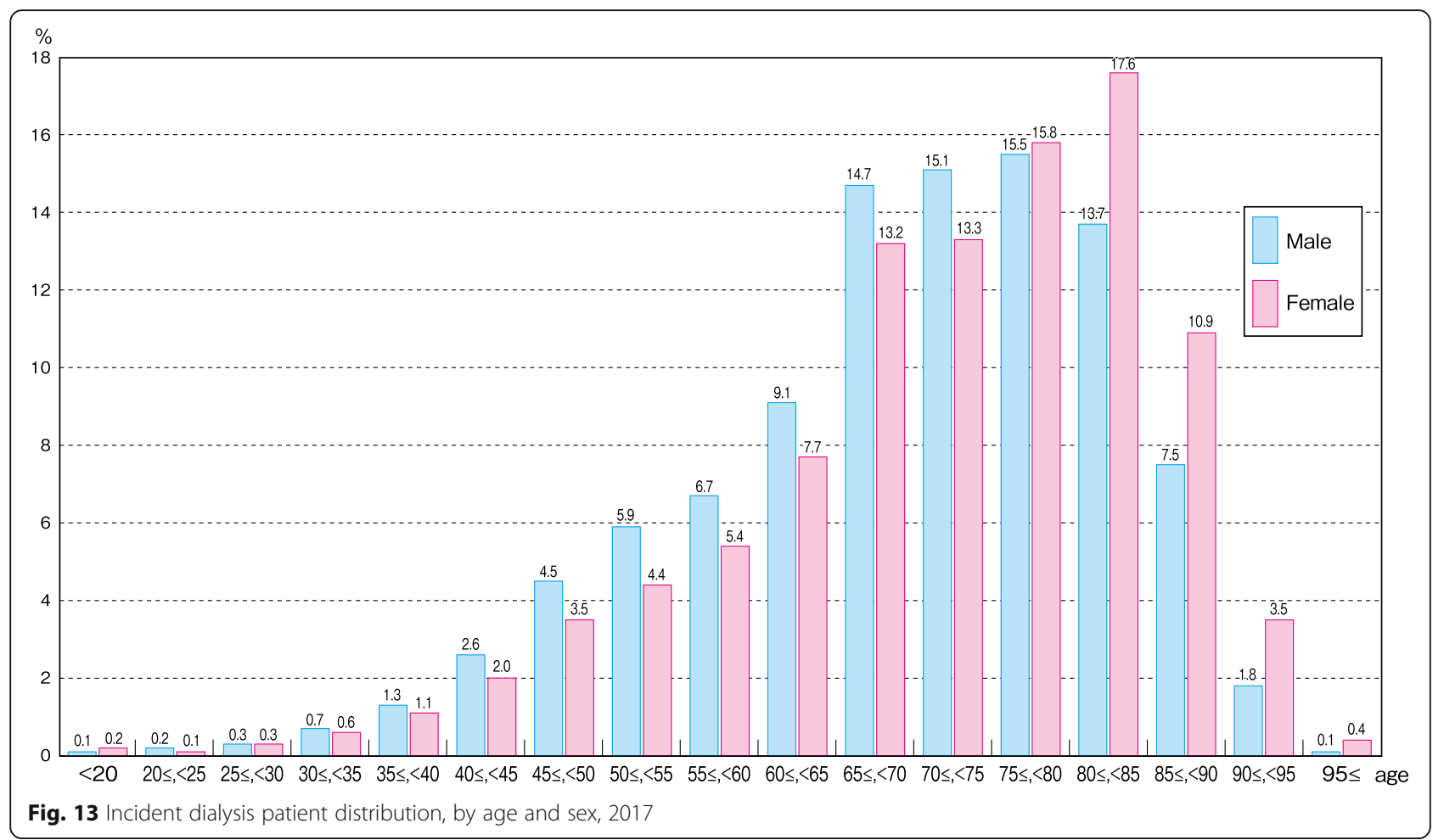

recommended for all dialysis treatments in the JSDT standard. These standards were the strictest in the world at the time they were established and remained so at the end of 2017.

As part of the revisions made to the medical care reimbursement system in Japan in 2010, an additional fee for the dialysis fluid standard was newly established, and this led to major improvements in the level of dialysis fluid standard management [7]. According to an analysis of dialysis fluid ET levels and dialysis patient prognosis that was conducted using the data from the 2015 survey, the group of patients that underwent treatment at facilities that maintained the dialysis fluid ET level at under $0.001 \mathrm{EU} / \mathrm{mL}$ had markedly higher 1-year survival rates than the group of patients who underwent treatment at facilities where the ET levels were at or above $0.100 \mathrm{EU} /$ $\mathrm{mL}$ [10]. Biological contamination of dialysis fluid was newly added to the 2017 survey, and as a result biochemical contamination and measures to prevent this type of contamination were newly surveyed.

The dialysis fluid standard management status data included in this chapter was calculated using data from facilities with at least one dialysis console, which totaled 4346 facilities in the 2017 survey.

\section{Dialysis fluid ET testing}

The dialysis fluid ET level test that is part of the JSDT standard is conducted using the limulus test $[8,9]$. In

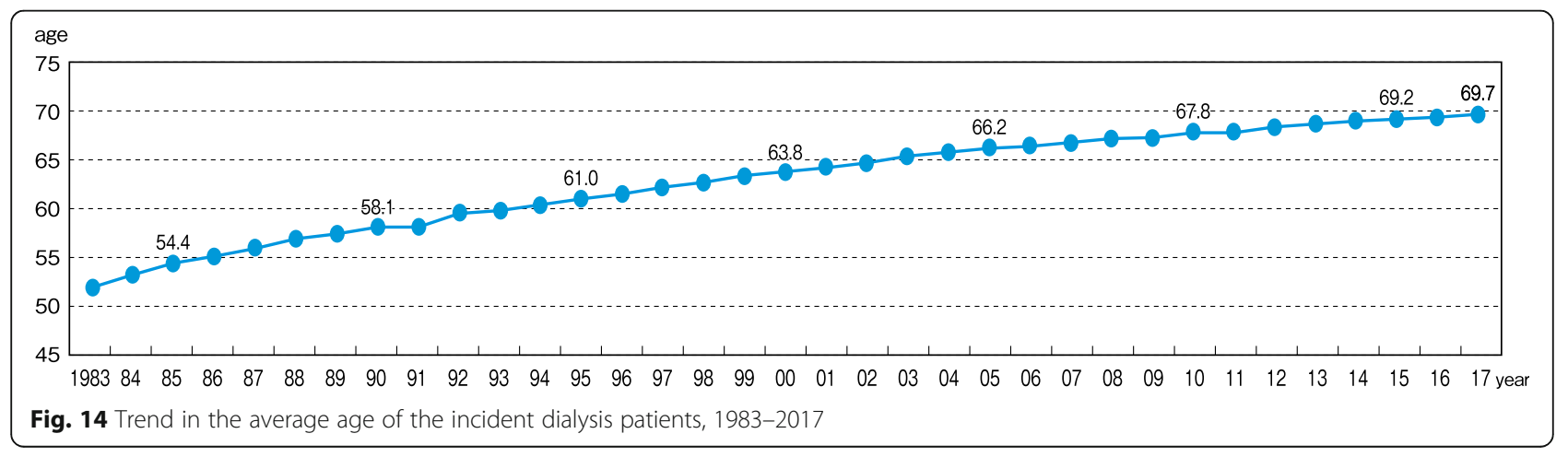



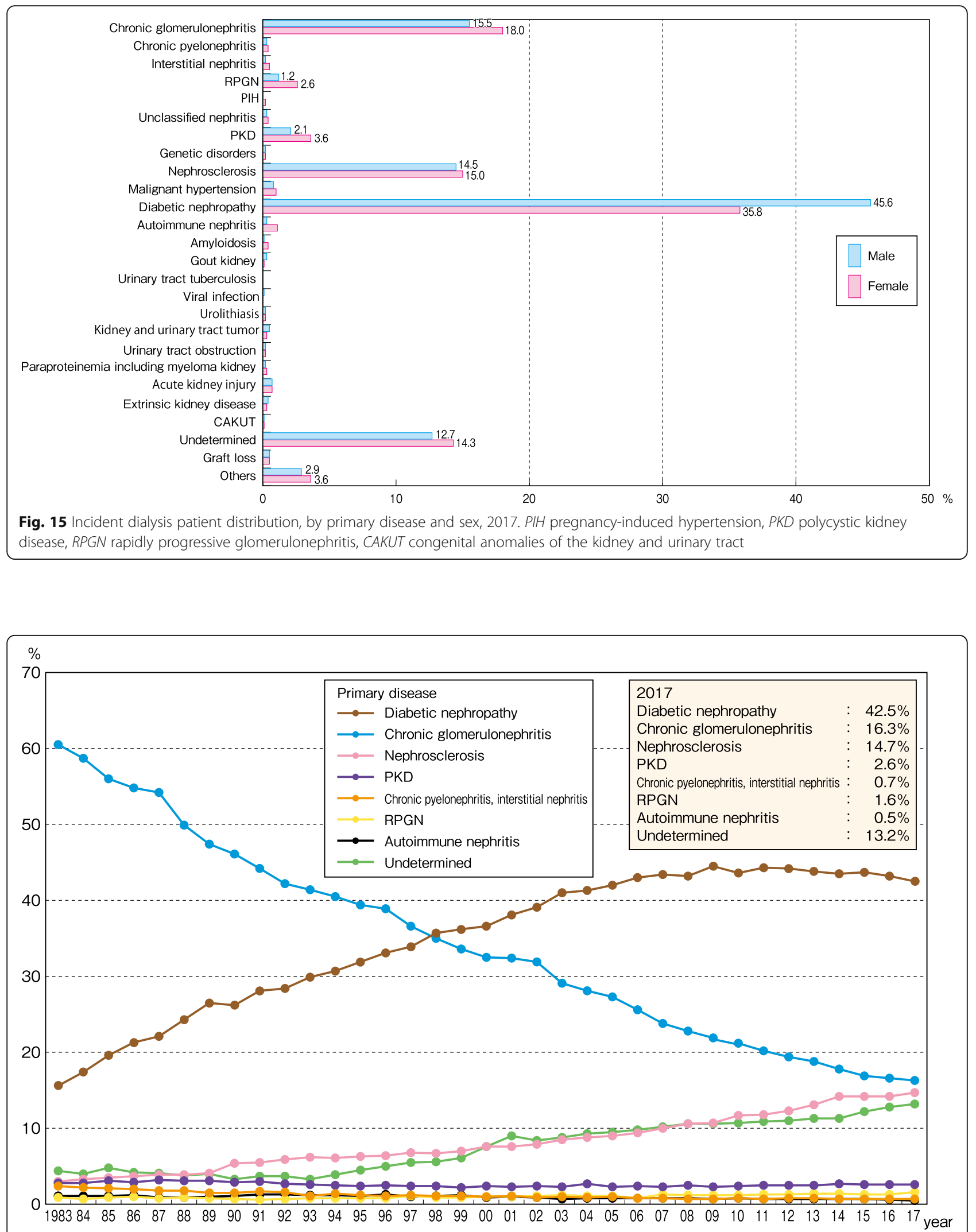

Fig. 16 Trends in major primary diseases of the incident dialysis patients, 1983-2017. PKD polycystic kidney disease, RPGN rapidly progressive glomerulonephritis 


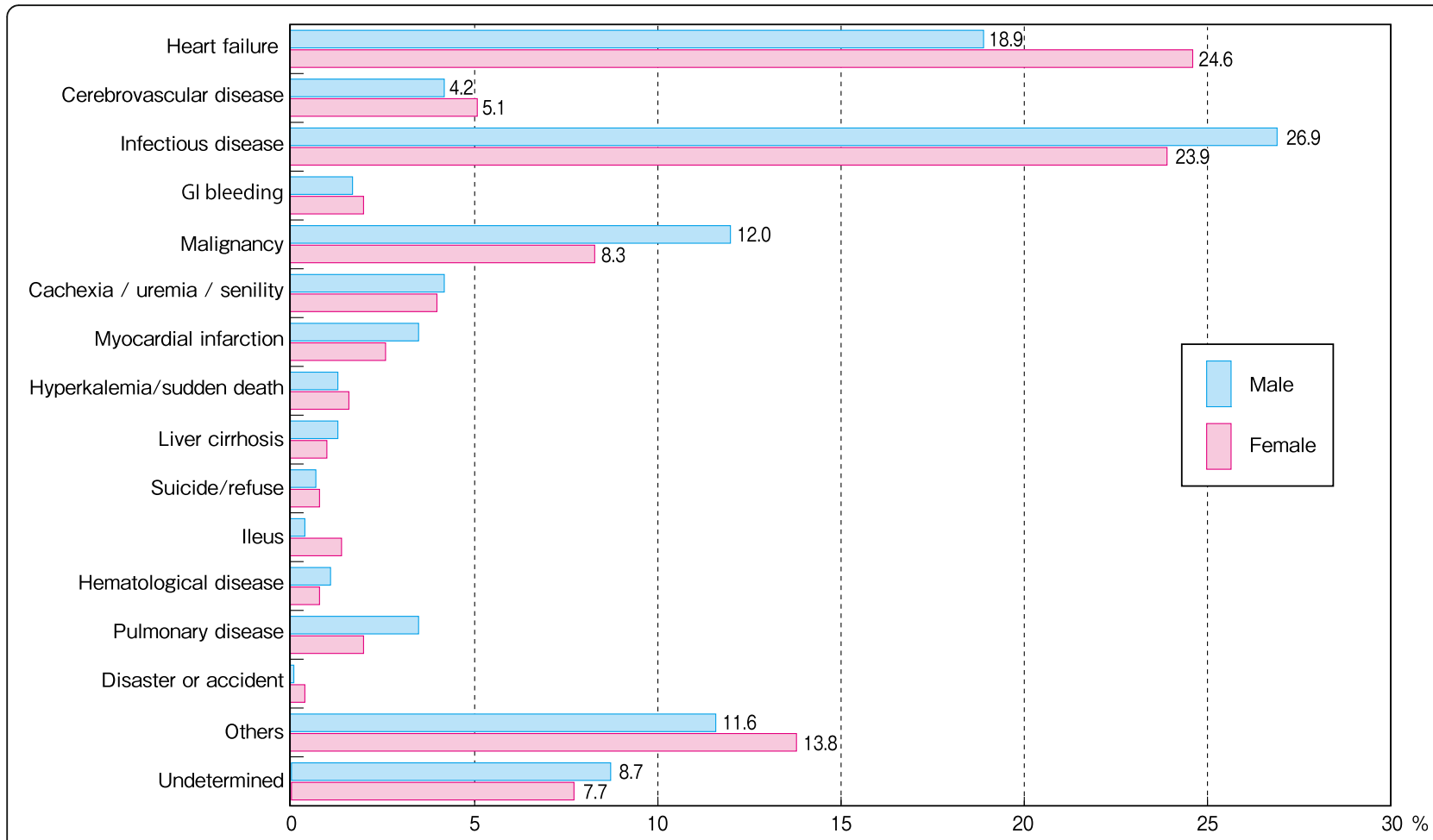

Fig. 17 Incident dialysis patient distribution, by cause of death and sex, 2017

Japan, several ET measurement machines are relatively inexpensive and available over-the-counter; thus, it is widely used by most dialysis facilities. However, it is quite rare in the rest of the world.

A total of 4305 facilities out of all surveyed facilities responded to the frequency of ET testing. The number of facilities that comply with the stipulated frequency of "at least once per month" was 3601 , which was $83.6 \%$ of the total (Fig. 19a, Additional file 19: Table S19). Observation of the annual changes in measurement frequency indicate that the percentage of facilities that performed the dialysis fluid ET test in 2008, the year the standard was implemented, was $33.1 \%$ but that this percentage drastically increased to $70.6 \%$ in 2010 , the year in which the dialysis fluid standard additional fee was newly established, and has consistently increased since then (Fig. 20a, Additional file 20: Table S20).

Responses regarding dialysis fluid ET levels were received from 4188 facilities, 3446 (82.3\%) of which indicated that they met the UPD standard of under 0.001 $\mathrm{EU} / \mathrm{mL}$ and 4046 (96.6\%) of which indicated that they met the standard for standard dialysis fluid of $0.050 \mathrm{EU} /$ mL (Fig. 19b, Additional file 19: Table S19). Observation of the chronological changes in dialysis fluid ET levels indicates that both the under $0.001 \mathrm{EU} / \mathrm{mL}$ and the under $0.050 \mathrm{EU} / \mathrm{mL}$ standard are increasing annually (Fig. 20b, Additional file 20: Table S20). The absence of values for dialysis fluid ET concentration in 2008 is due to the switch in the unit of dialysis fluid ET concentration from $\mathrm{EU} / \mathrm{L}$ to $\mathrm{EU} / \mathrm{mL}$ based on international rules in the survey that year, resulting in many incorrect entries.

\section{Dialysis fluid TVC testing}

According to the standard, the results of dialysis fluid bacterial culturing are to be assessed as follows: The dialysis fluid TVC is the number of colonies identified 7 days after culturing at between 17 and $23{ }^{\circ} \mathrm{C}$ using heterotrophic agar plate medium [8, 9]. A total of 4289 facilities responded to the question regarding the frequency with which dialysis fluid TVC is measured. Of those, 3488 facilities reported testing at least once per month, which represents $81.3 \%$ of all facilities (Fig. 21a, Additional file 21: Table S21). The frequency of TVC measurement increasing annually, and although it increased markedly in 2010 (as was the case for ET testing), in all other years the frequency has been slightly lower than that for ET testing (Fig. 22a, Additional file 22: Table S22).

A total of 4072 facilities responded to the question regarding dialysis fluid TVC. Of these, 3129 facilities (76.8\% overall) reported meeting the UPD standard of $0.1 \mathrm{cfu} / \mathrm{mL}$ and 4031 facilities (99.0\%) reported meeting the standard dialysis fluid standard of $100 \mathrm{cfu} / \mathrm{mL}$ (Fig. 21b, Additional file 21: Table S21). The percentage of facilities meeting the UPD standard and the percentage of those meeting the standard dialysis 

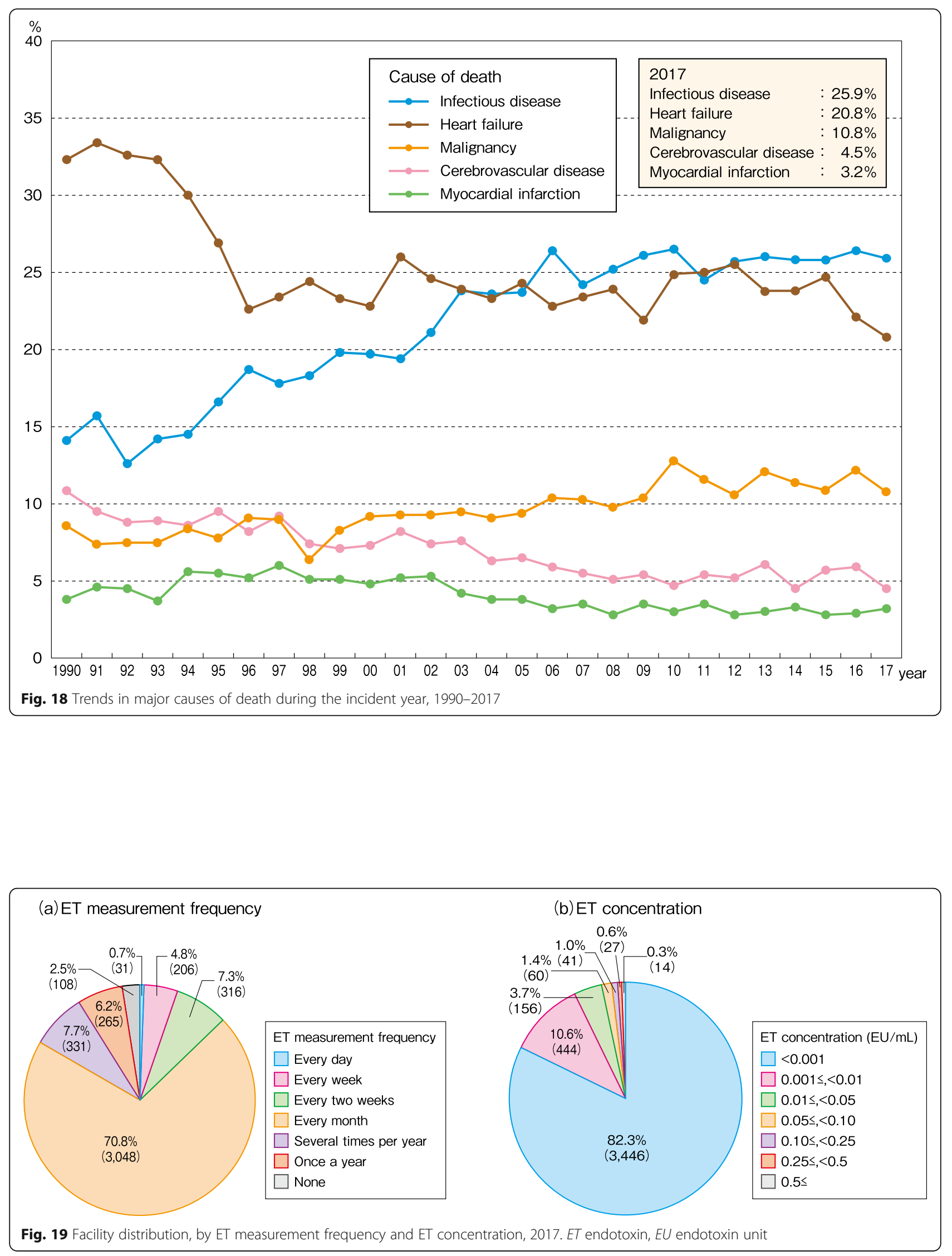


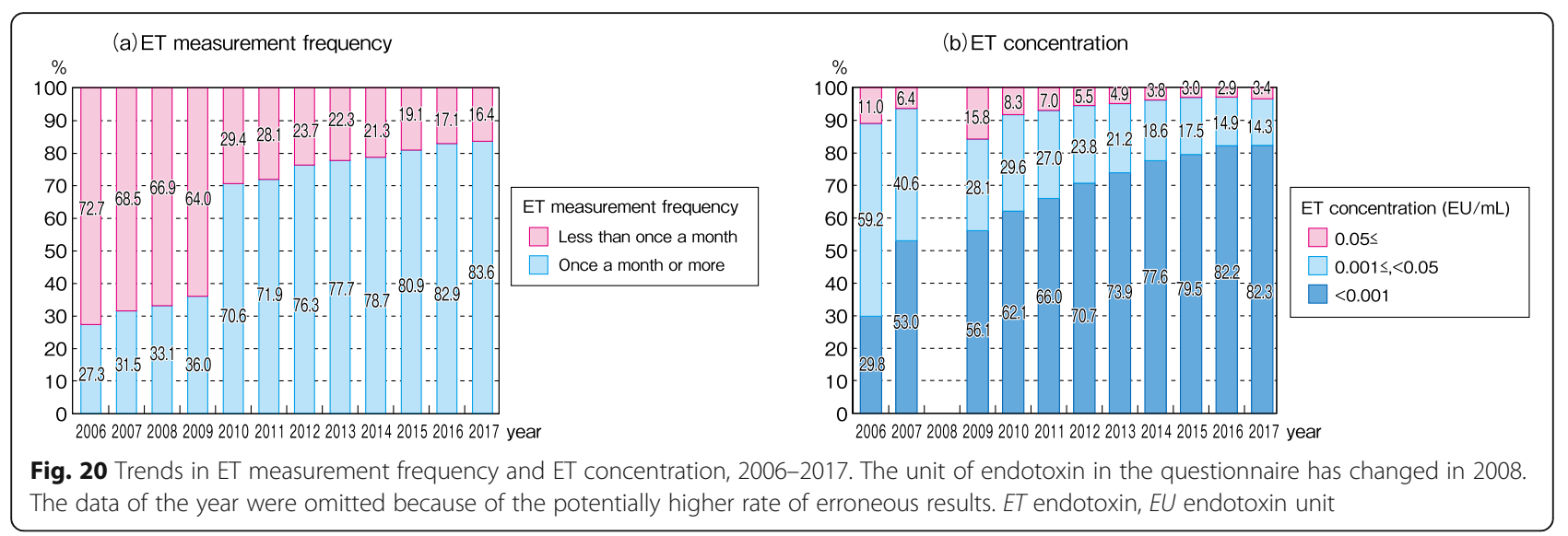

fluid are increasing annually (Fig. 22b, Additional file 22: Table S22).

\section{Achievement quotient of UPD and standard dialysis fluid}

As the JSDT standard stipulates the bacteriological standard for dialysis fluid (both UPD and standard dialysis fluid), the numerical values for both dialysis fluid ET concentration and TVC must be simultaneously met $[8,9]$. The number of facilities that responded to the questions about both dialysis fluid ET level and TVC was 4062. Of these, 2942 facilities $(72.4 \%$ overall) reported meeting the UPD standard (dialysis fluid ET level of under $0.001 \mathrm{EU} /$ $\mathrm{mL}$ and live bacteria count of under $0.1 \mathrm{cfu} / \mathrm{mL}$ ) and 3912 facilities reported meeting the standard for standard dialysis fluid (dialysis fluid ET level of under $0.050 \mathrm{EU} / \mathrm{mL}$ and TVC of under $100 \mathrm{cfu} / \mathrm{mL}$; Fig. 23, Additional file 23: Table S23). The achievement quotients for both UPD and standard dialysis fluid have been increasing over time, which suggests that in Japan the dialysis fluid purity level is increasing (Fig. 24, Additional file 24: Table S24).

\section{Source of dialysis water and chemical contamination preventative measures}

A total of 4306 facilities responded to the question regarding the source of dialysis water that was included in the 2017 survey. The most common source was tap water, which was reported by 3668 facilities (85.2\%). This was followed by ground water (377 facilities, $8.8 \%$ ) and the blend of tap water and ground water (251 facilities, 5.8\%; Fig. 25, Additional file 25: Table S25).

A total of 4267 facilities responded to the question regarding the frequency of residual chlorine testing before the treatment. Of these, "every day" was the most common response (2377 facilities, 55.7\%), followed by "once per week" (927 facilities, 21.7\%) and "once per month" (225 facilities, 5.3\%; Fig. 26a, Additional file 26: Table S26). A total of 510 facilities $(12.0 \%$ overall) reported that they do not measure (a) TVC measurement frequency

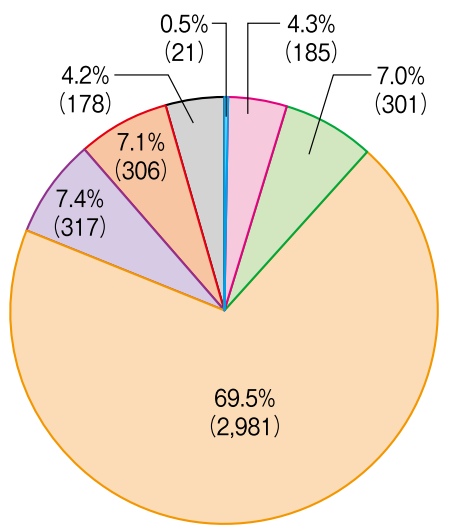

TVC measurement frequency

Every day

$\square$ Every week

$\square$ Every two weeks

$\square$ Every month

$\square$ Several times per year

$\square$ Once a year

$\square$ None (b) TVC

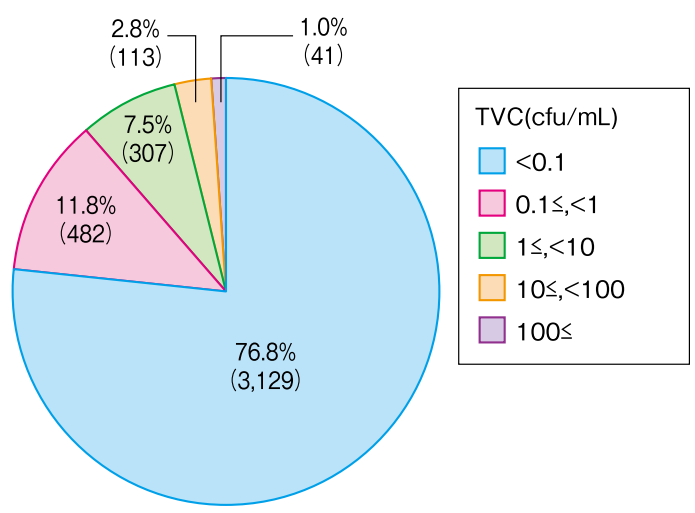

Fig. 21 Facility distribution, by TVC measurement frequency and TVC, 2017. TVC total viable microbial count, cfu colony forming unit 


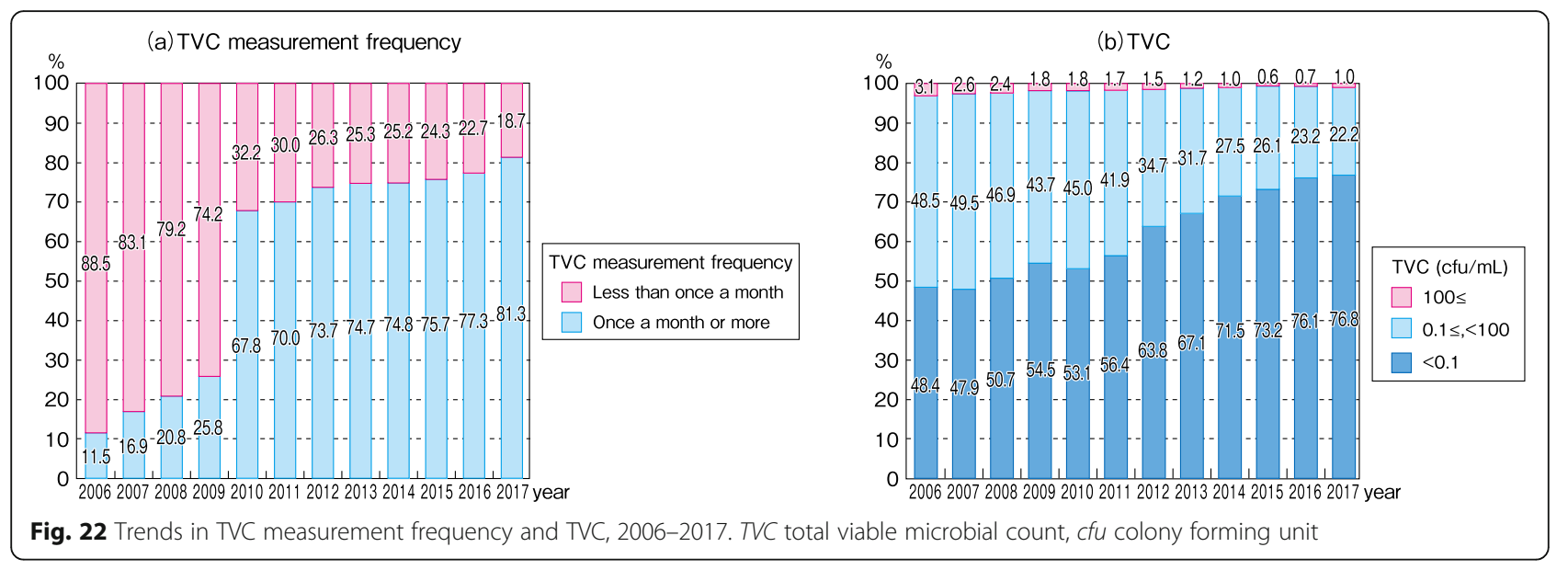

residual chlorine. This issue requires further study, including investigation of the measurement frequency. A total of 3965 facilities responded to the question regarding the residual chlorine measurement method, with most (1812, 45.7\%) reporting "free chlorine only," followed by 1275 facilities (32.2\%) that reported using both free chlorine and total chlorine. A total of 799 facilities (20.2\%) reported using total chlorine only (Fig. 26b, Additional file 26: Table S26).

A total of 4242 facilities reported familiarity with the JSDT chemical contamination standard [9], with $81.4 \%$ overall reporting either "very familiar" or "familiar" (Fig. 27a, Additional file 27: Table S27). A total of 4106 facilities responded to the question regarding the frequency with which chemical contamination is measured, as is stipulated by the standard. Overall, 1544 facilities (37.6\%) reported "once per year" while 1348 facilities (32.8\%) reported that they do not measure chemical contamination (Fig. 27b, Additional file 27: Table S27). Although the chemical contamination standard for dialysis fluid is relatively well-known, however, not many facilities actually measured chemical contamination, and thus we should promote routine measurement of chemical contamination.

\section{Chapter 5: prescription of HD and HDF Current status of HDF}

HDF includes the following modalities: On-line HDF, off-line HDF, push/pull HDF, acetate-free biofiltration

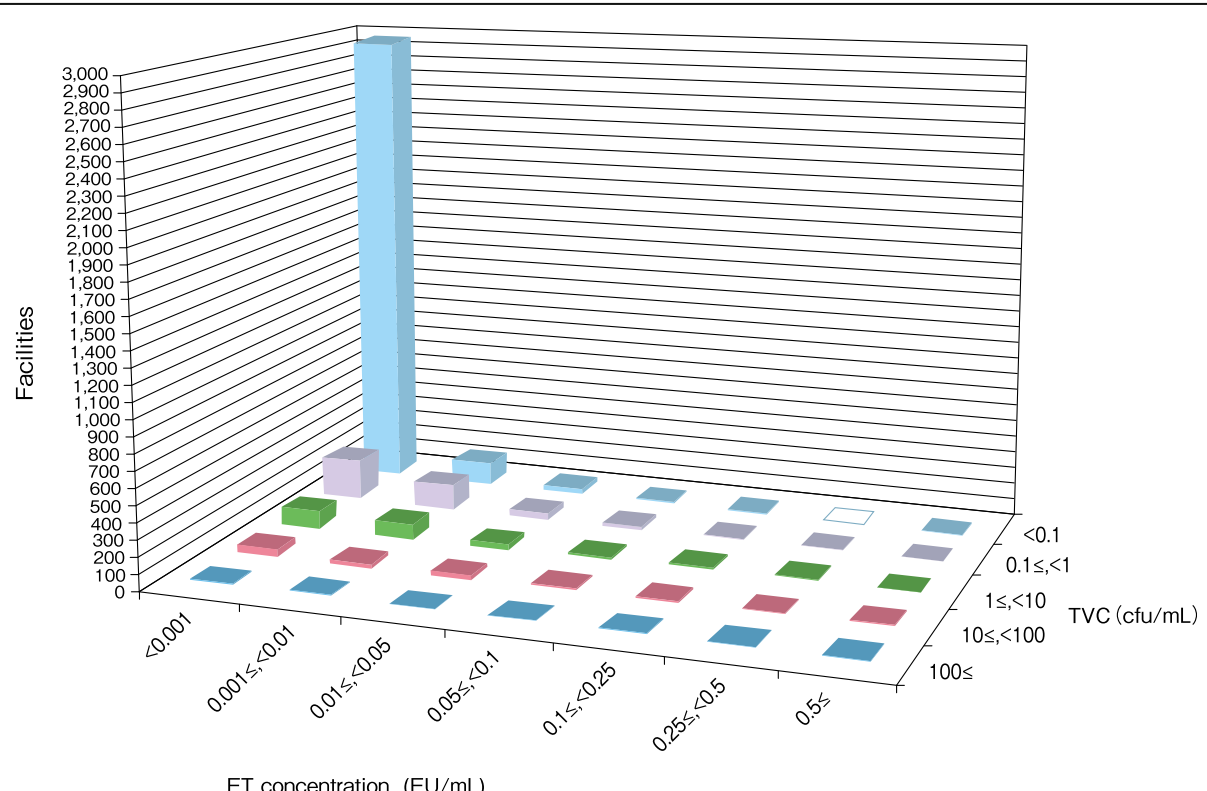

Fig. 23 Facility distribution, by ET concentration and TVC, 2017. ET endotoxin, EU endotoxin unit, TVC total viable microbial count, cfu colony forming unit 


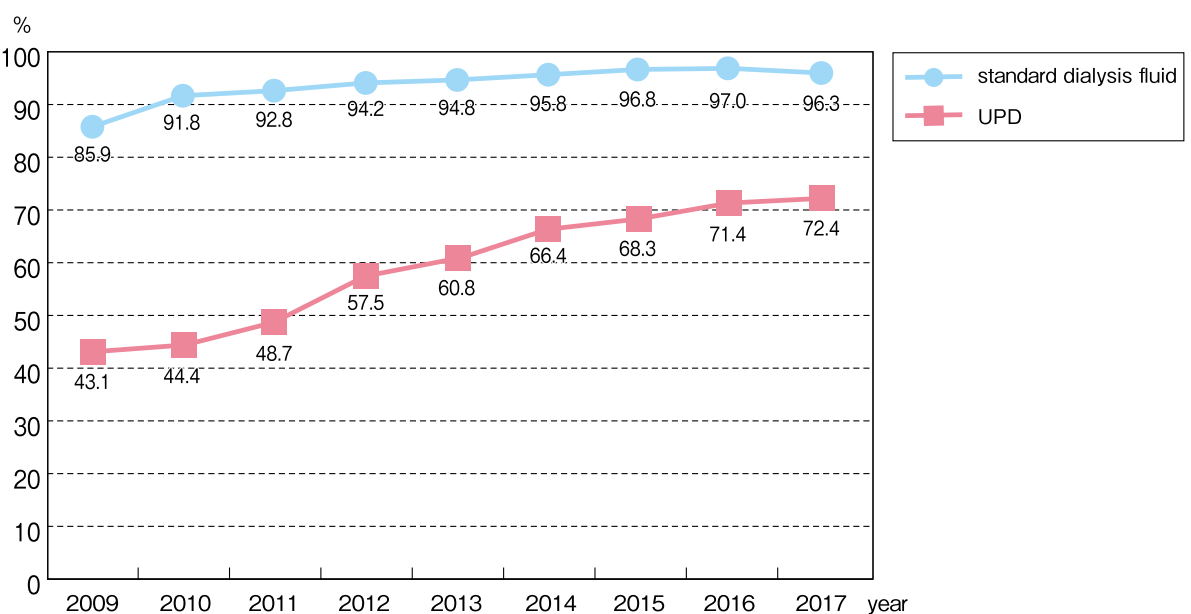

Fig. 24 Trends in facility distribution for achieving UPD and standard dialysis fluid, 2009-2017. UPD ultrapure dialysis fluid

(AFBF), and intermittent infusion hemodiafiltration (IHDF).

In Japan, the number of HDF patients has been rapidly increasing since 2012. Totals calculated using facility survey data indicate that at the end of 2017, the number had reached 95,140 (+18,304 compared to the previous year), which accounts for $29.4 \%$ of all HD and HDF patients (+ 5.3 points as compared to the previous year; Table 5).

There were 91,948 HDF patients from the patient survey, of whom 70,604 (the most numerous group) were undergoing on-line HDF (76.8\% of HDF patients), followed by IHDF at 17,105 patients (18.6\% of HDF patients; Fig. 28, Additional file 28: Table S28).

The mean age of HDF patients was 66.6 years overall (males: 65.9 years, females: 68.0 years), whereas the mean age of HD patients was 69.4 years overall, approximately 3 years older (males: 68.6 years, females: 71.0 years; Fig. 29, Additional file 29: Table S29).
The mean dialysis vintage for HDF patients was 8.8 years overall (males: 8.1 years, females: 10.1 years). The percentage of patients with a dialysis vintage of under 5 years was high, at $39.4 \%$ overall (males: $41.9 \%$, females: $34.7 \%)$. The mean dialysis vintage of HD patients was 6.8 years overall (males: 6.3 years, females: 7.5 years). The percentage of patients with a dialysis vintage of under 5 years was $50.0 \%$ overall (males: $52.1 \%$, females: 46.2\%; Fig. 30, Additional file 30: Table S30). HDF patients in Japan have longer dialysis vintages than HD patients, and HDF is indicated for relatively young patients.

\section{Comparison of the prescription of HD and HDF Membrane material}

In the 2017 survey, dialysis prescriptions were surveyed in detail, as was done in the 2008 survey. A total of 195, $883 \mathrm{HD}$ patients and $82,436 \mathrm{HDF}$ patients responded to

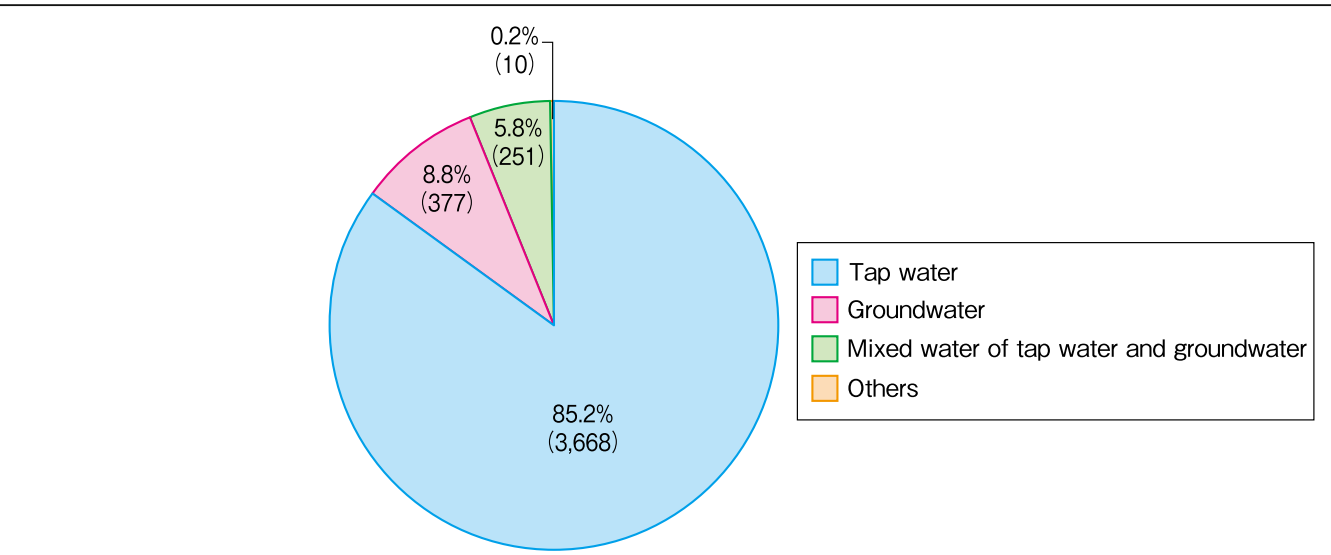

Fig. 25 Facility distribution, by source of dialysis water 
(a) Measurement frequency for residual chlorine

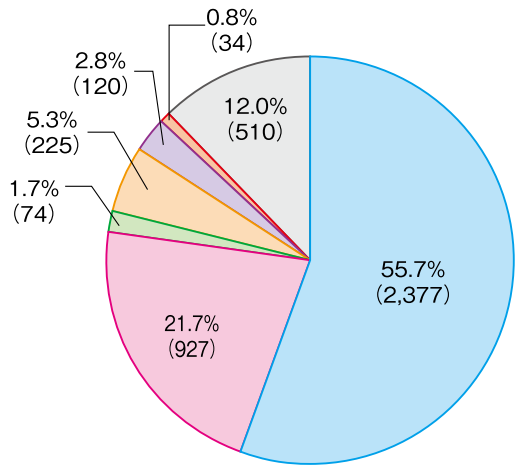

Measurement frequency for residual chlorine

$\square$ Every day

$\square$ Once a week

$\square$ Several times per month

$\square$ Once a month

$\square$ Several times per year

$\square$ Once a year

$\square$ None (b) Measurement method for residual chlorine

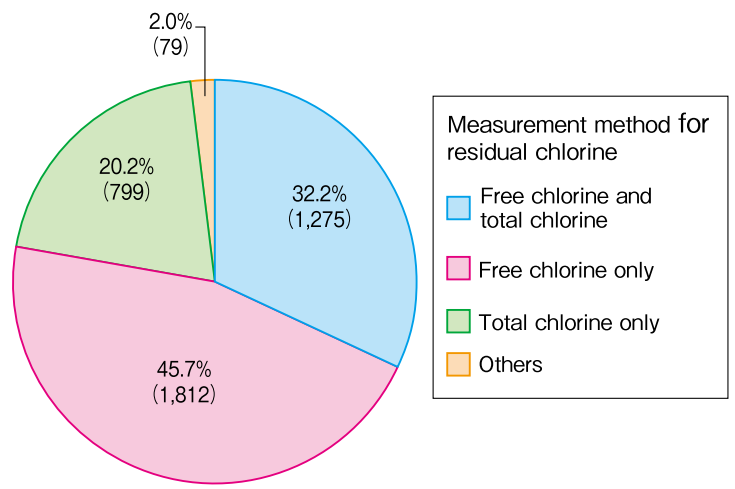

Fig. 26 Facility distribution, by measurement frequency for residual chlorine and measurement method

the question regarding membrane material. The most common membrane material used by HD patients was polysulfone (PS), at $56.5 \%$. This was followed by polyethersulfone (PES) at $16.4 \%$, cellulose triacetate (CTA) at $15.6 \%$, polymethylmethacrylate (PMMA) at $4.1 \%$, and polyether polymer alloy (PEPA) at $3.1 \%$. Observation of the statistics for all HDF patients indicates that $43.5 \%$ used PS, 36.3\% used PES, $14.3 \%$ used CTA, and $4.6 \%$ used PEPA (Fig. 31, Additional file 31: Table S31).

A total of 60,830 on-line HDF patients and 3226 offline HDF patients responded to the questions regarding HDF modality and membrane material. Of the on-line HDF patients, patients using PS with pre-dilution accounted for $41.8 \%$ and those using PS with postdilution accounted for $43.4 \%$, the latter of which was the most numerous groups, followed by PES with predilution at $36.9 \%$ and PES with post-dilution at $32.9 \%$. Of the off-line patients, those using PS with pre-dilution accounted for $42.9 \%$ and those using PS with postdilution accounted for $52.4 \%$, the latter of which was the most numerous group (as was the case with on-line HDF), followed by PES with pre-dilution at $36.4 \%$ and PES with post-dilution at $33.1 \%$. Overall, 15,385 IHDF patients responded to the question regarding membrane material. PS was most frequently used at $46.5 \%$, followed by PES at 36.7\% (Fig. 32, Additional file 32: Table S32).

\section{Dialyzer category}

A total of 195,883 HD patients and 82,436 HDF patients responded to the question regarding the dialyzer category. Of the HD patients, $55.2 \%$ used type Ia, 33.1\% used type IIa, $5.6 \%$ used type $\mathrm{S}, 2.2 \%$ used type IIb, and $2.0 \%$ used plate type polyacrylonitrile (PAN). Nearly all the HDF patients (96.1\%) used hemodiafilter (Fig. 33, Additional file 33: Table S33). (a) Awareness of the JSDT standard for chemical contaminants

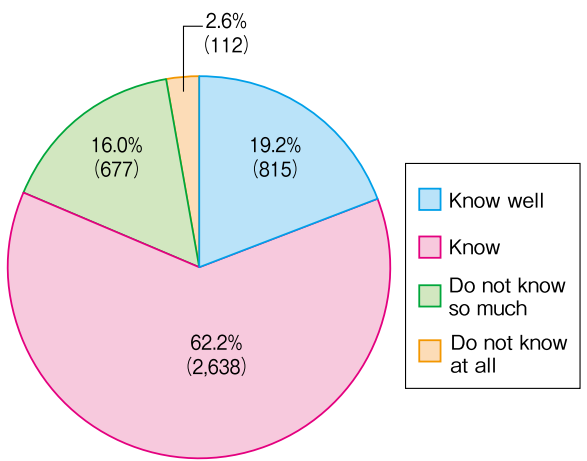

(b) Measurement frequency for chemical contamination

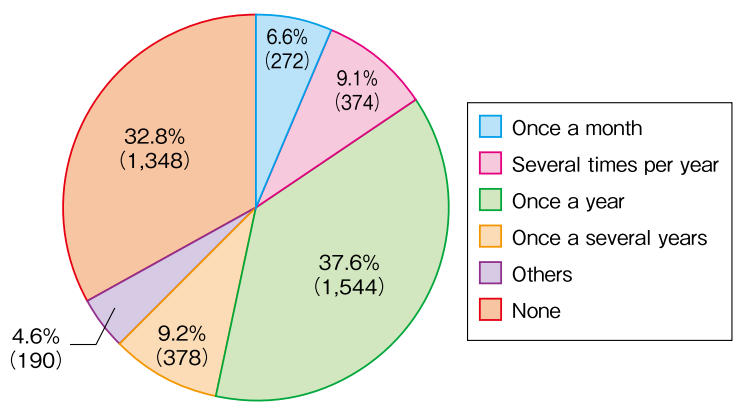

Fig. 27 Facility distribution, by awareness of the JSDT standard for chemical contaminants and measurement frequency. JSDT the Japanese Society for Dialysis Therapy 


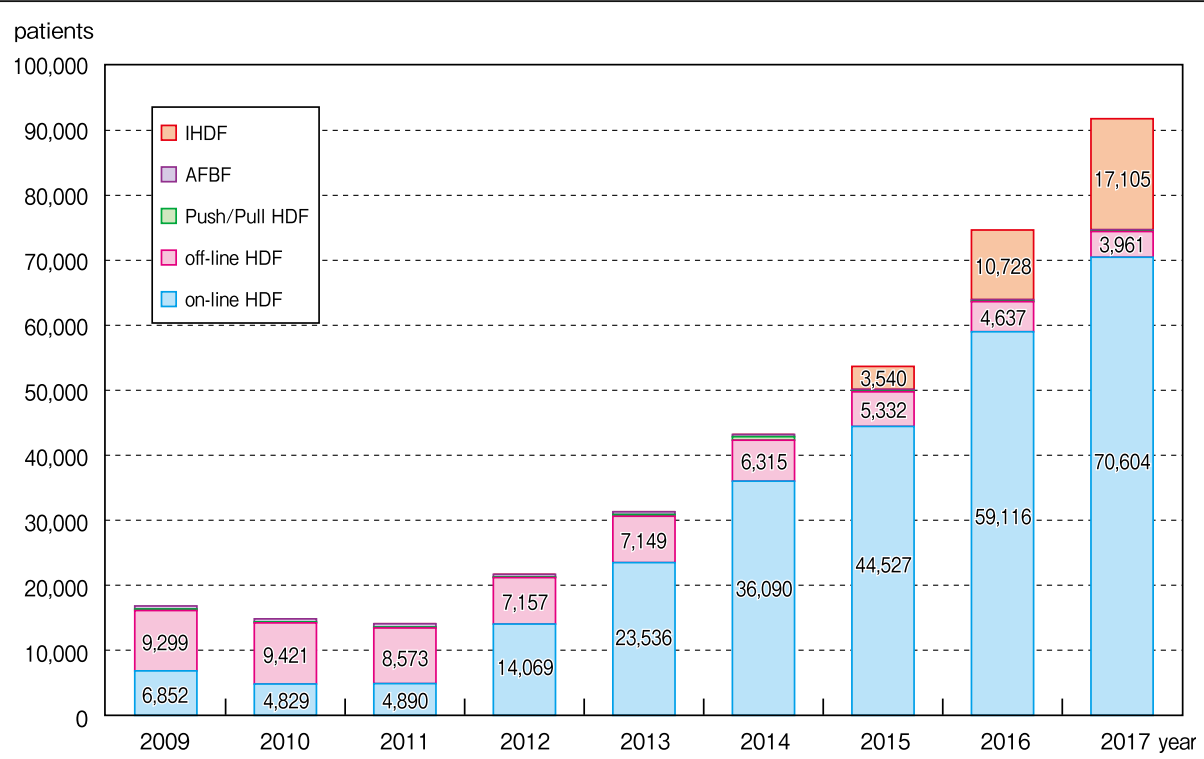

Fig. 28 HDF patient count, by HDF modality, 2009-2017. AFBF acetate free biofiltration, HDF hemodiafiltration, IHDF intermittent infusion hemodiafiltraiton

\section{Membrane surface area}

A total of 195,317 patients responded to the question regarding the dialyzer membrane surface area used in HD. The mean HD membrane surface area was $1.75 \mathrm{~m}^{2}$, with the category of "1.4 $\mathrm{m}^{2} \leq,<1.6 \mathrm{~m}^{2}$ " the largest at $27.4 \%$, followed by " $2.0 \mathrm{~m}^{2} \leq,<2.2 \mathrm{~m}^{2}$ " at $26.4 \%$. A total of 82,237 patients responded to the question regarding the dialyzer membrane surface area used in HDF. The mean HDF membrane surface area was $1.96 \mathrm{~m}^{2}$, with the category of "2.0 $\mathrm{m}^{2} \leq,<2.2 \mathrm{~m}^{2}$ " the largest at $34.4 \%$, followed by " 1.4 $\mathrm{m}^{2} \leq,<1.6 \mathrm{~m}^{2}$ " at 20.2\% (Fig. 34, Additional file 34: Table S34). Larger membrane surface areas were used in HDF treatment.

A total of 60,682 on-line HDF patients and 3230 offline HDF patients responded to the questions regarding the HDF modality and the membrane surface area. The mean membrane surface area for on-line HDF patients overall was $2.00 \mathrm{~m}^{2}$ (pre-dilution: $2.02 \mathrm{~m}^{2}$, post-dilution: $1.97 \mathrm{~m}^{2}$ ). The largest category for both pre- and postdilution on-line HDF patients was " $2.0 \mathrm{~m}^{2} \leq,<2.2 \mathrm{~m}^{2}$,"
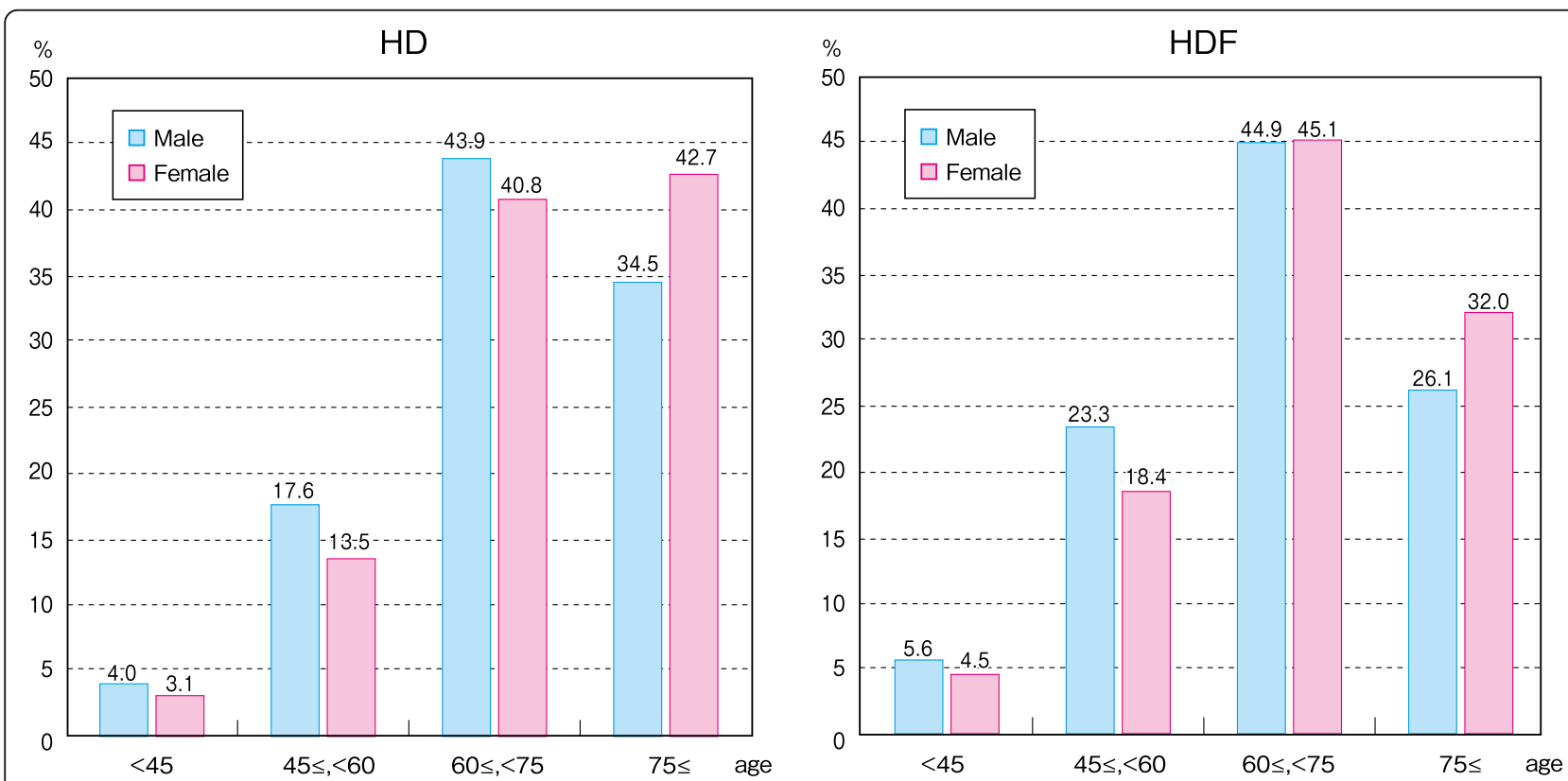

Fig. 29 HD HDF patient distribution, by age and sex, 2017. HD hemodialysis, HDF hemodiafiltration 


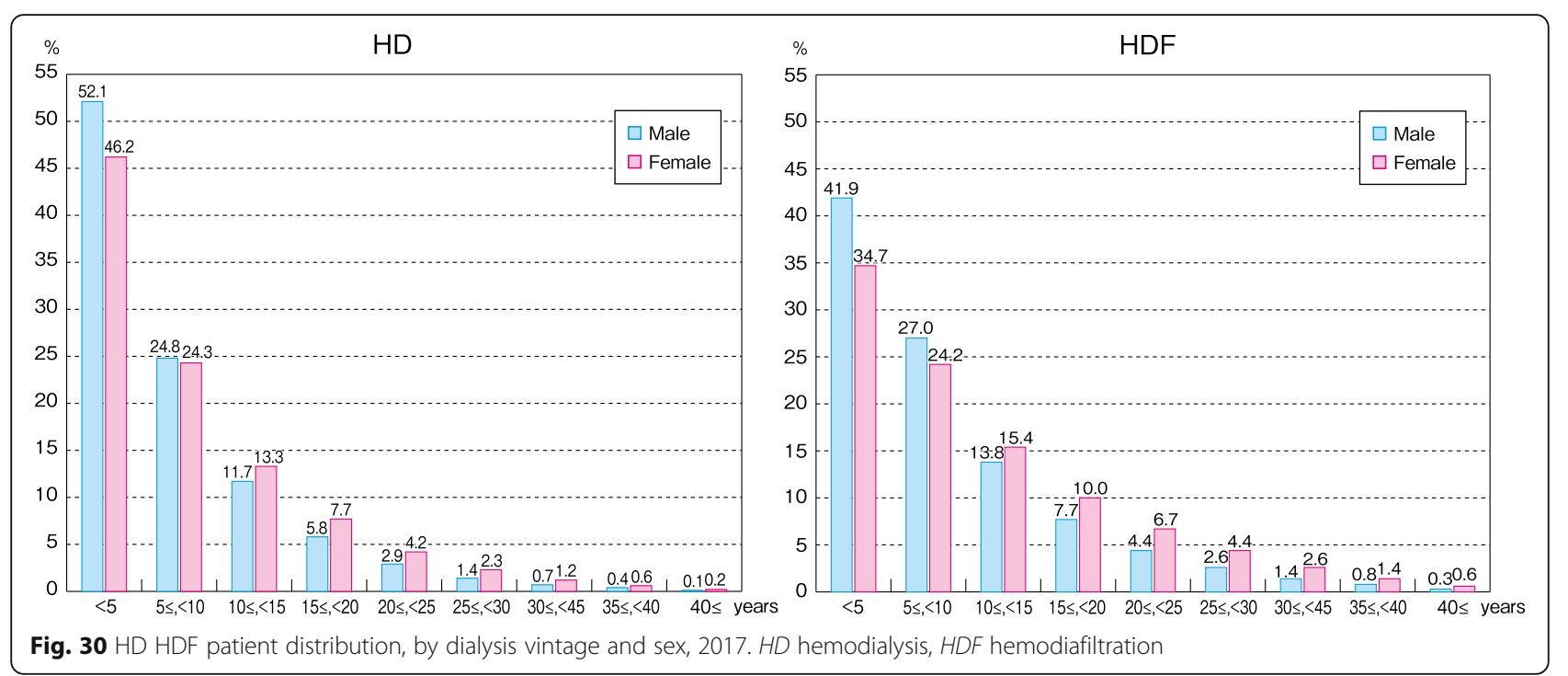

followed by "1.4 $\mathrm{m}^{2} \leq,<1.6 \mathrm{~m}^{2}$." The mean membrane surface area for off-line HDF patients overall was 1.82 $\mathrm{m}^{2}$ (pre-dilution: $1.76 \mathrm{~m}^{2}$, post-dilution: $1.83 \mathrm{~m}^{2}$ ). The most numerous size category for pre-dilution off-line HDF patients was " $1.4 \mathrm{~m}^{2} \leq,<1.6 \mathrm{~m}^{2}$ " at $30.1 \%$, followed by "2.0 $\mathrm{m}^{2} \leq,<2.2 \mathrm{~m}^{2}$ " at $27.8 \%$ (Fig. 35, Additional file 35: Table S35).

\section{Dialysis time}

A total of 203,009 HD patients and 85,928 HDF patients responded to the question regarding dialysis time. The mean dialysis time was $238.7 \mathrm{~min}$ for $\mathrm{HD}$ patients and $243.2 \mathrm{~min}$ for HDF patients, and this trend was the same as 2009 [11]. For both groups, the "240 $\mathrm{min} \leq,<270 \mathrm{~min}$ " group had the most patients, with $67.9 \%$ of the HD patients and $69.3 \%$ of the HDF patients (Fig. 36, Additional file 36: Table S36).

\section{Blood flow rate}

A total of 200,825 HD patients and 85,108 HDF patients responded to the question regarding blood flow rate. The mean blood flow rate was $206 \mathrm{~mL} / \mathrm{min}$ for HD patients and $224 \mathrm{~mL} / \mathrm{min}$ for HDF patients, indicating that the HDF group had a higher blood flow rate. The figures from 2009 were $197 \mathrm{~mL} / \mathrm{min}$ for $\mathrm{HD}$ and $211 \mathrm{~mL} / \mathrm{min}$ for HDF, indicating an increase of approximately $10 \mathrm{~mL} / \mathrm{min}$ for both groups [11].The blood flow rate category with the highest number of patients in both groups was " $200 \mathrm{~mL} /$ $\min \leq,<220 \mathrm{~mL} / \mathrm{min}$ " at $44.1 \%$ for HD and $34.0 \%$ for HDF. In the HDF group, which had a large number of

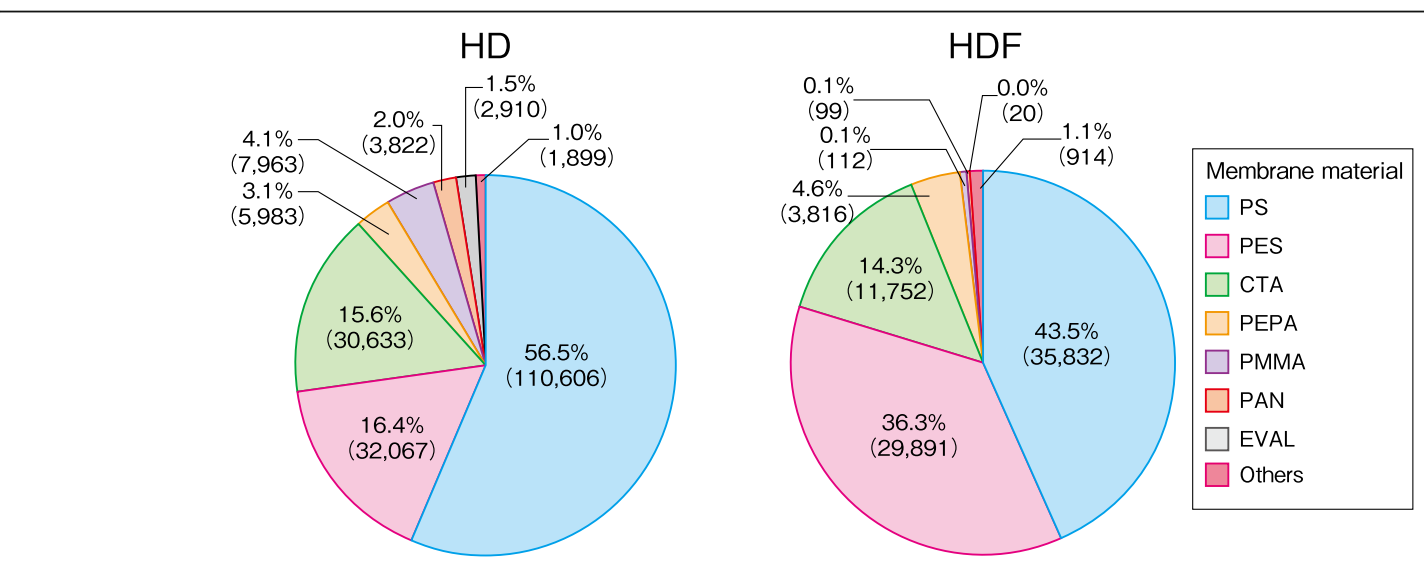

Fig. 31 HD HDF patient distribution, by membrane material, 2017. HD hemodialysis, HDF hemodiafiltration, PS polysulfone, PES polyethersulfone, CTA cellulose triacetate, PEPA polyether polymer alloy, PMMA polymethylmethacrylate, PAN polyacrylonitrile, EVAL ethylene vinylalcohol copolymer 


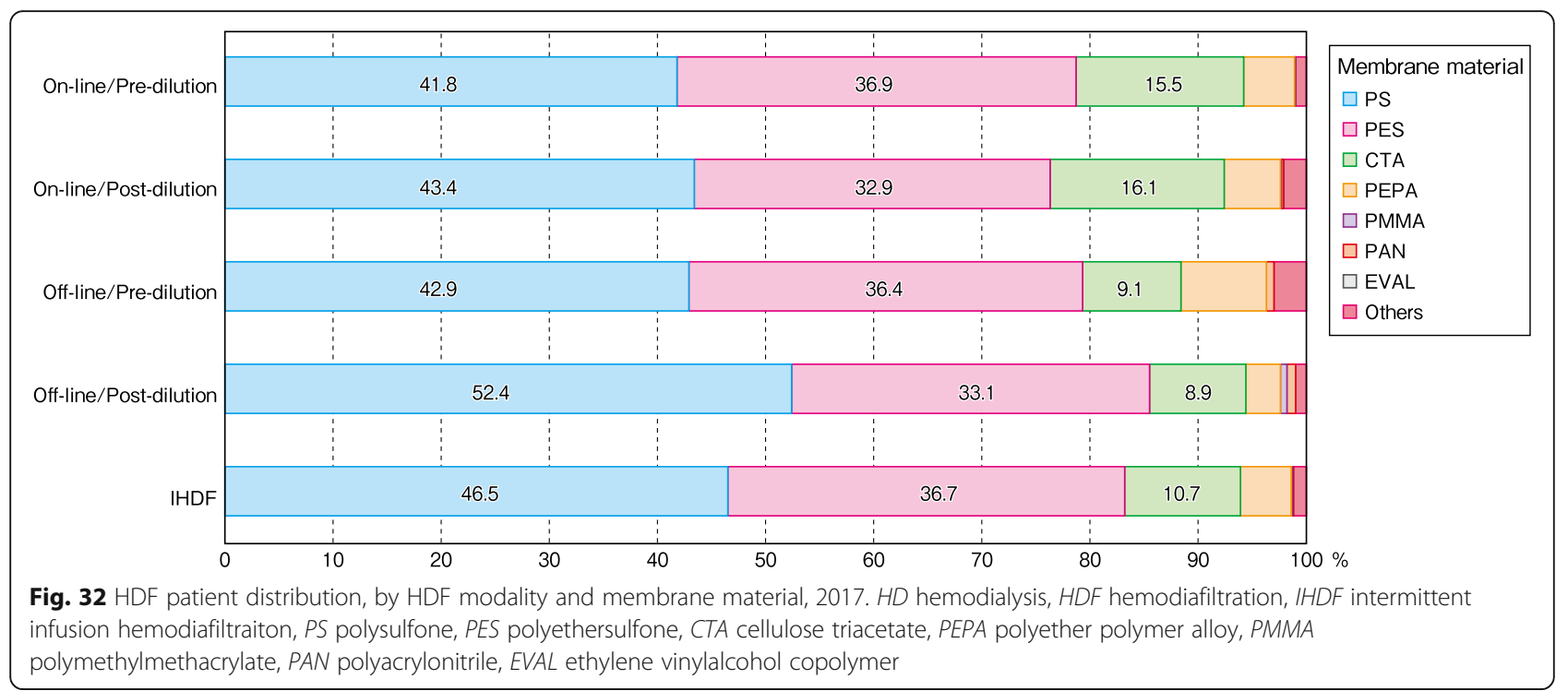

patients, $23.6 \%$ had high blood flow rates of " $240 \mathrm{~mL} / \mathrm{min}$ $\leq,<260 \mathrm{~mL} / \mathrm{min}$ " (Fig. 37, Additional file 37: Table S37).

\section{B2-MG kinetics}

In the 2017 survey, the kinetics of $\beta 2$-MG, which is recognized as an important marker for assessing recent dialysis modalities, was evaluated by comparing the pre- vs. post-dialysis $\beta 2$-MG levels and the $\beta 2-$ MG removal rate. The JSDT guidelines for dialysis prescriptions recommend that the pre-dialysis serum $\beta 2$-MG level be under $30 \mathrm{mg} / \mathrm{L}$ and if possible, under $25 \mathrm{mg} / \mathrm{L}$ [12]

The $\beta 2-\mathrm{MG}$ removal rate was calculated as shown below:

$\beta 2-\mathrm{MG}$ removal rate $(\%)=\{($ pre-dialysis $\beta 2-\mathrm{MG}$ level post-dialysis $\beta 2$-MG level) / pre-dialysis $\beta$ 2-MG level $\} \times 100$

A total of $158,791 \mathrm{HD}$ patients and 70,535 HDF patients responded to the question regarding pre-dialysis $\beta 2-\mathrm{MG}$ concertation. The mean pre-dialysis $\beta 2-\mathrm{MG}$ concertation was equivalent by the modality, $27.0 \mathrm{mg} / \mathrm{L}$ in HD patients and $27.1 \mathrm{mg} / \mathrm{L}$ in HDF patients (Fig. 38, Additional file 38: Table S38).

A total of 155,022 HD patients indicated both their pre-dialysis $\beta 2-\mathrm{MG}$ concentration and their dialyzer category. The mean pre-dialysis $\beta 2-\mathrm{MG}$ concentration was $26.7 \mathrm{mg} / \mathrm{L}$ for type Ia, $25.4 \mathrm{mg} / \mathrm{L}$ for type $\mathrm{Ib}, 27.1 \mathrm{mg} / \mathrm{L}$ for type IIa, $27.5 \mathrm{mg} / \mathrm{L}$ for type IIb, and $30.0 \mathrm{mg} / \mathrm{L}$ for plate type AN69 (Fig. 39, Additional file 39: Table S39). Of the HD patients, a total of 52,500 on-line HDF patients and 2743 off-line HDF patients indicated both their pre-dialysis $\beta 2-\mathrm{MG}$ concentration and their HDF modality. The mean values for the treatment modalities in both groups were pre-dilution: $27.0 \mathrm{mg} / \mathrm{L}$, on-line post-dilution: $27.3 \mathrm{mg} / \mathrm{L}$, off-line pre-dilution: $28.3 \mathrm{mg} /$ L, off-line post-dilution: $28.4 \mathrm{mg} / \mathrm{L}$, and IHDF: $27.1 \mathrm{mg} /$ L (Fig. 40, Additional file 40: Table S40).
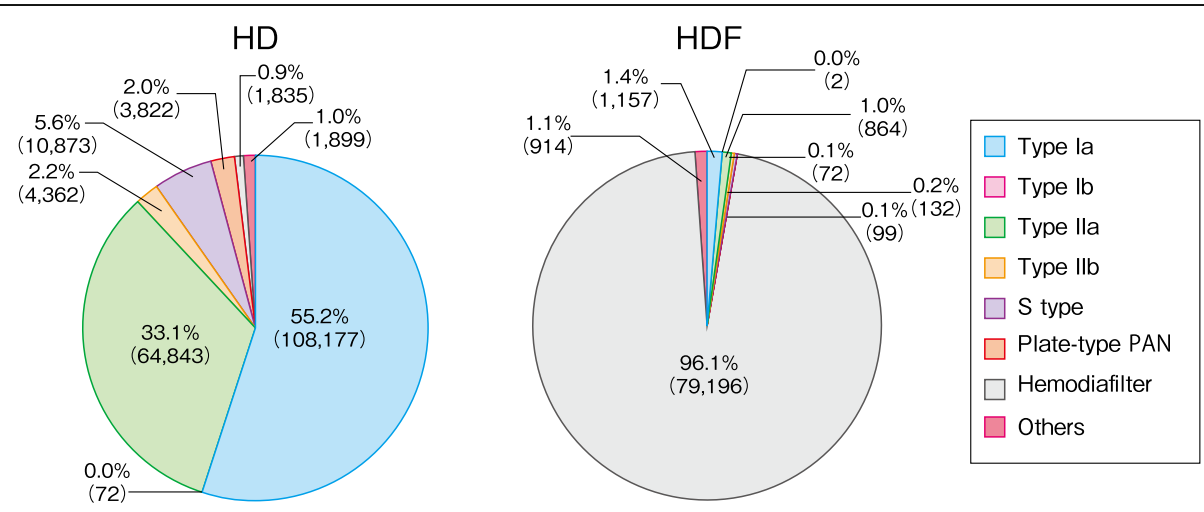

Fig. 33 HD HDF patient distribution, by dialyzer category, 2017. HD hemodialysis, HDF hemodiafiltration, PAN polyacrylonitrile 

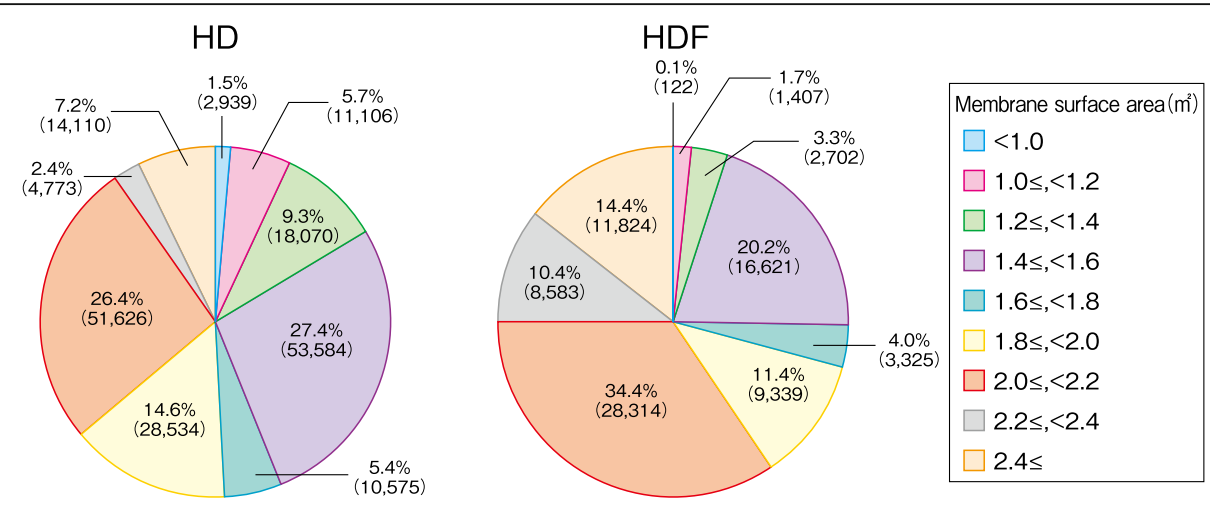

Fig. 34 HD HDF patient distribution, by membrane surface area, 2017. HD hemodialysis, HDF hemodiafiltration

The achievement quotient for the $30 \mathrm{mg} / \mathrm{L}$ target in the JSDT guideline was 70.5\% (HD: 70.1\%, HDF: 71.5\%), while the achievement quotient for the $25 \mathrm{mg} / \mathrm{L}$ target was $36.9 \%$ (HD: $37.1 \%$, HDF: 35.6\%).

A total of 46,203 HD patients and 23,415 HDF patients responded to the question regarding $\beta 2-\mathrm{MG}$ removal rate. The mean removal rate was $60.7 \%$ for HD patients overall and $71.4 \%$ for HDF patients overall, indicating that HDF patients overall had a higher mean value than HD patients overall (Fig. 41, Additional file 41: Table S41).

A total of 45,346 HD patients responded when indicating their dialyzer category that their $\beta 2-\mathrm{MG}$ removal rate could be calculated. The mean values by functional category were $59.1 \%$ for type Ia, $57.8 \%$ for type $\mathrm{Ib}, 67.2 \%$ for type IIa, $68.7 \%$ for type IIb, $43.7 \%$ for type S, and $32.4 \%$ for plate type AN69 (Fig. 42, Additional file 42: Table S42). Of the HDF patients, 17,892 on-line HDF patients and 837 off-line HDF patients responded when indicating their HDF dilution method that their $\beta 2-\mathrm{MG}$ removal rate could be calculated. The mean values for the treatment modalities were on-line pre-dilution: $73.0 \%$, on-line postdilution: $72.9 \%$, off-line pre-dilution: $63.9 \%$, off-line postdilution: $69.6 \%$, and the value for IHDF patients overall was $65.0 \%$ (Fig. 43, Additional file 43: Table S43).

\section{Chapter 6: peritoneal dialysis}

The facility survey totals indicate that at the end of 2017, there were 9090 peritoneal dialysis (PD) patients (+69 patients as compared to the previous year) (Table 5). Among them 7325 patients underwent PD alone, and 1505 underwent the combination therapy with $\operatorname{HD}(\mathrm{F})$ (hybrid therapy) once per week, 155 underwent the combination of twice per week, 37 underwent the combination of three times per week, and 68 were undergoing other combined therapy. The survey for the number of incident PD patients was started in 2015 in the facility survey, and the number in 2017 was 2117 (+171 as compared to the previous year; Fig. 44, Additional file 44: Table S44).

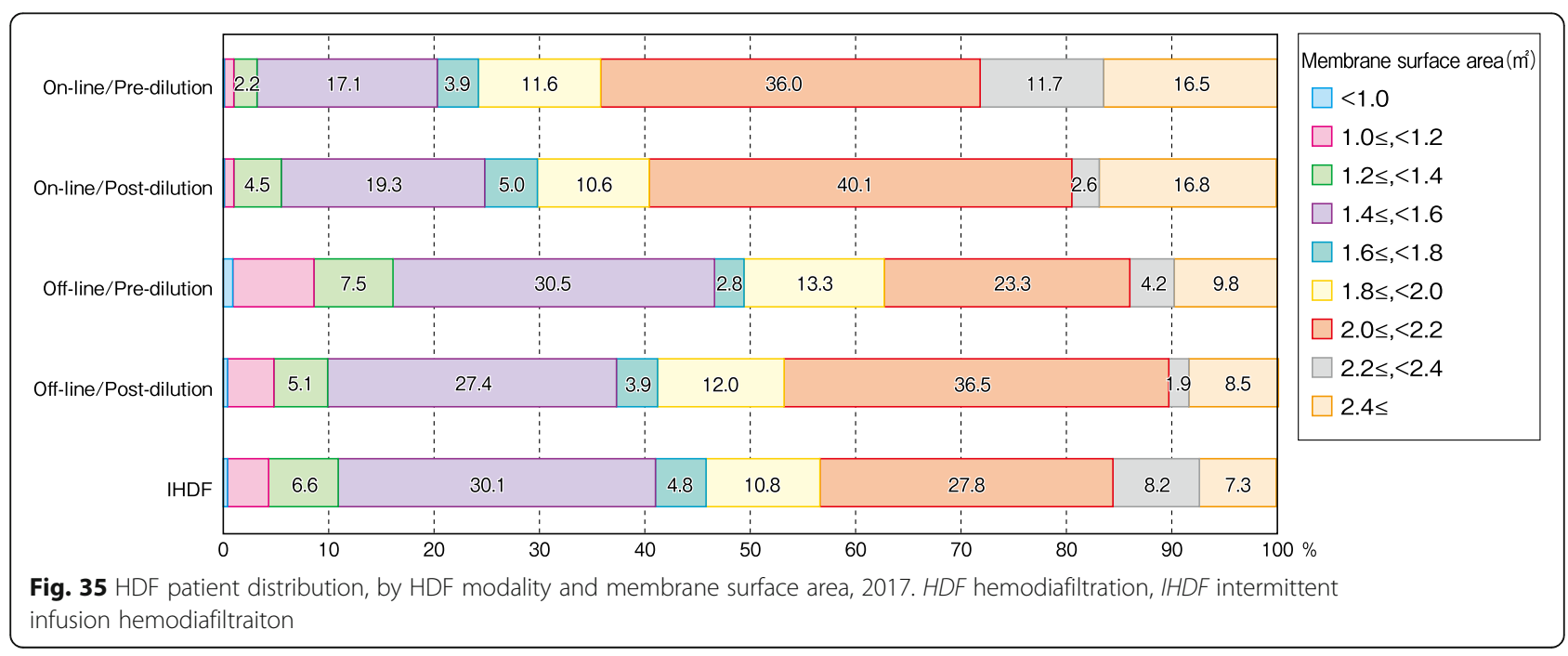




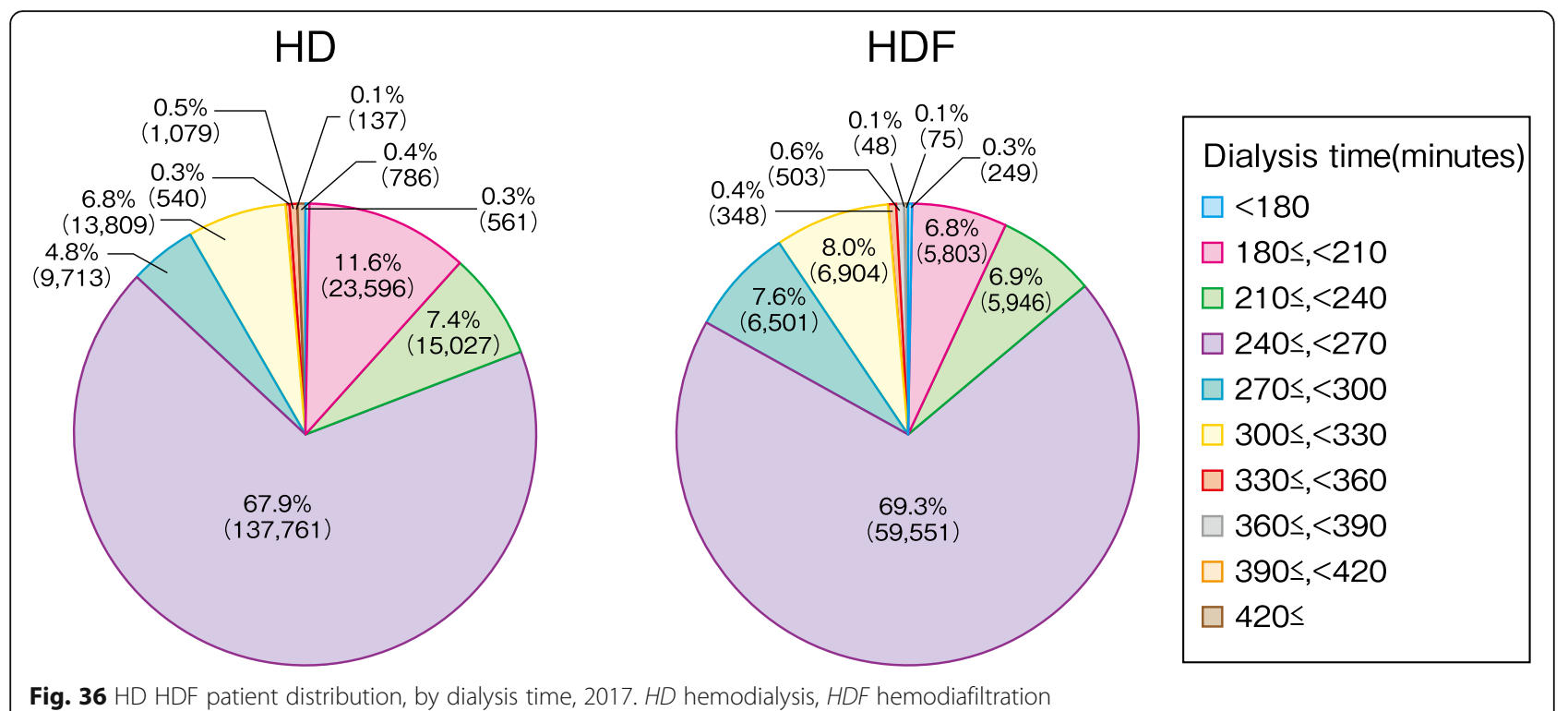

Fig. 36 HD HDF patient distribution, by dialysis time, 2017. HD hemodialysis, HDF hemodiafiltration

The patient survey totals indicate that of the 8669 PD patients who indicated both their sex and their age, $65 \%$ were male and $34.5 \%$ were female (Fig. 45 , Additional file 45: Table S45).

A total of 5958 patients indicated their PD vintage and their sex. The mean PD vintage overall was 3.3 years (males: 3.0 years, females: 3.7 years). The percentage of PD patients whose PD vintage was under 2 years was $43.9 \%$ overall (males: $46.3 \%$, females: $39.3 \%$ ), indicating that a large number of PD patients were in this category. The percentage of PD patients with a long-term continuous PD vintage of eight or more years was $8.0 \%$ overall (males: 6.6\%, females:
10.8\%; Fig. 46, Additional file 46: Table S46). A total of 5778 patients responded to the questions regarding dialysis fluid and PD vintage. The percentage of patients using only $1.5 \%$ glucose solution decreases as the PD vintage increases. The number of patients who indicated that they use either $1.5 \%$ or $2.5 \%$ glucose solution only as well as their PD vintage was 2904 (50.3\%), while the number who used $4.25 \%$ glucose solution only was only $6(0.1 \%)$. The number of patients who used icodextrin PD solution was 2868 (49.6\%; Fig. 47, Additional file 47: Table S47).

Of the 5696 patients who responded to the question regarding peritonitis rate, 4942 patients (86.8\%) indicated

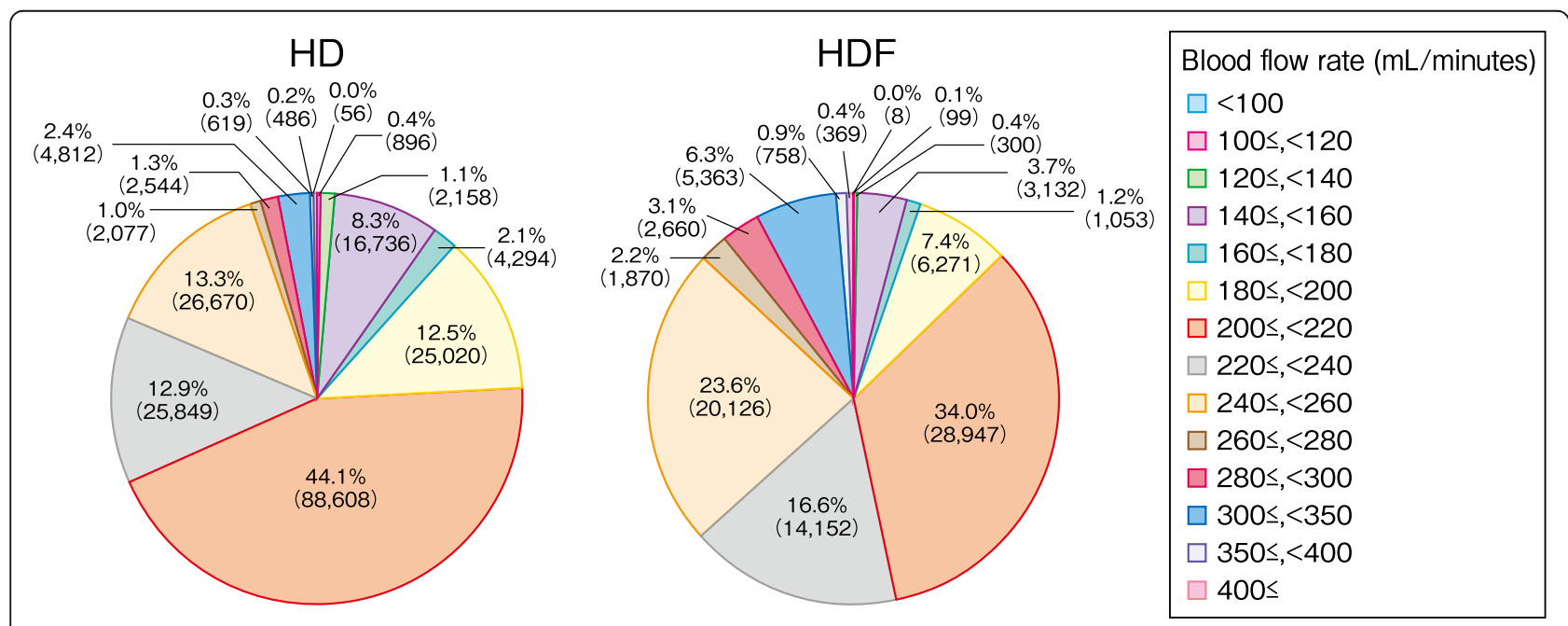

Fig. 37 HD HDF patient distribution, by blood flow rate, 2017. HD hemodialysis, HDF hemodiafiltration 


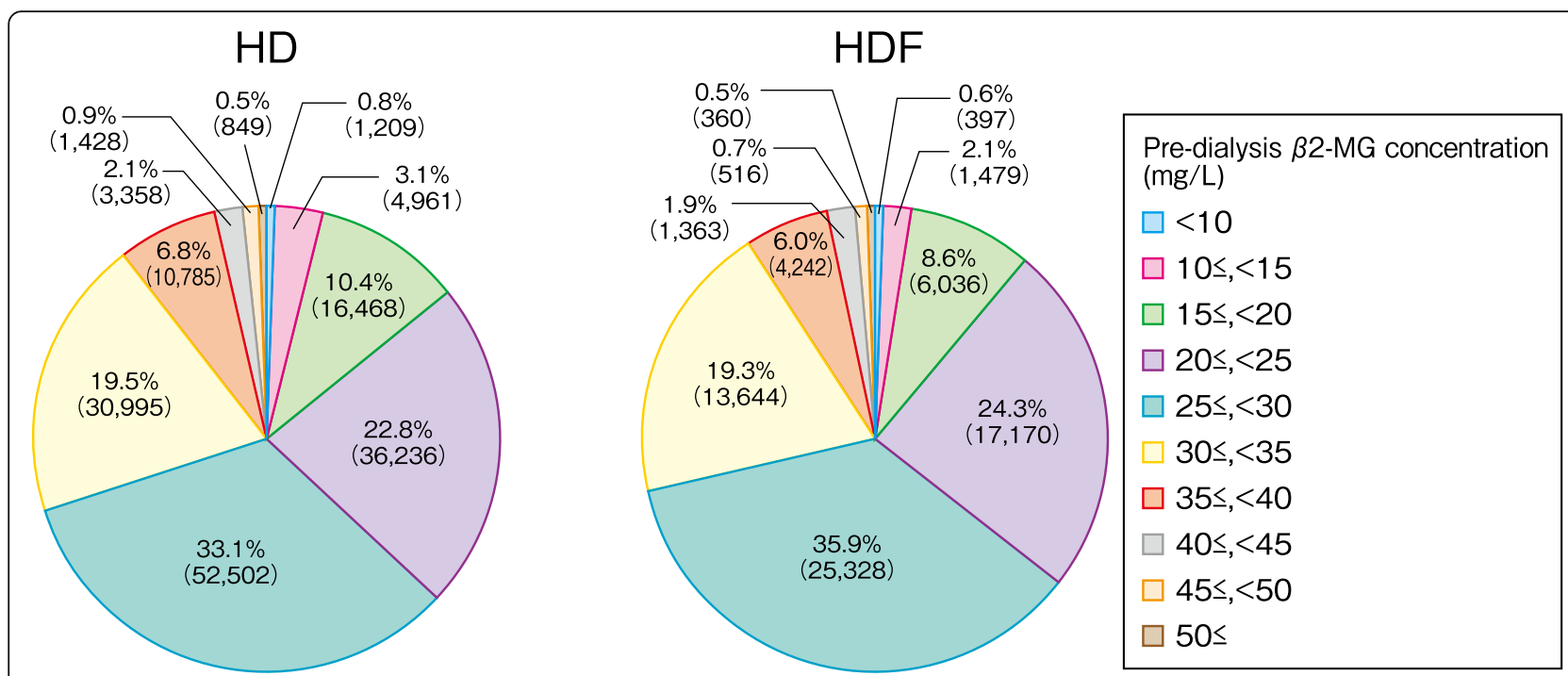

Fig. 38 HD HDF patient distribution, by pre-dialysis $\beta 2-M G$ concentration, 2017. $\beta 2-M G$ beta2-microglobulin, HD hemodialysis, HDF hemodiafiltration

that they did not experience peritonitis even once during 2017 (Fig. 48, Additional file 48: Table S48).

A total of 5638 patients responded to the questions regarding the peritonitis rate and the PD vintage.

Peritonitis rate was calculated as shown below:

Peritonitis rate $=$ no. of peritonitis onsets during 2017 / (no. of months of PD during 2017 / 12)
The overall peritonitis rate was 0.20 times/1 patient/ year (1 time/60.0 patients/month), which is far lower than the value recommended by the International Society for Peritoneal Dialysis (ISPD) guideline (0.50 times/1 patient/year) [13]. Observation of the data by PD vintage indicates that the highest peritonitis rate was among patients with a PD vintage of under 1 year at 0.26 times/ 1

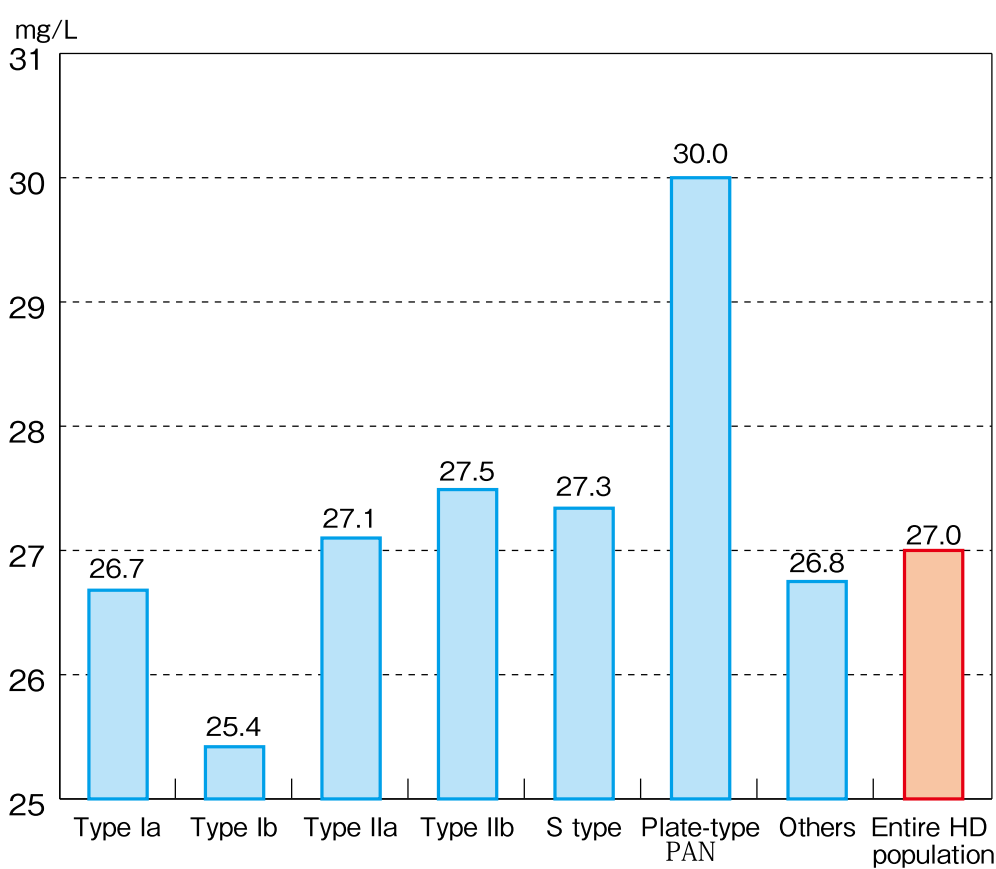

Fig. 39 Mean Pre-dialysis $\beta 2-M G$ concentration in HD patients, by dialyzer category, 2017. $\beta 2-M G$ beta2-microglobulin, HD hemodialysis, HDF hemodiafiltration, PAN polyacrylonitrile 


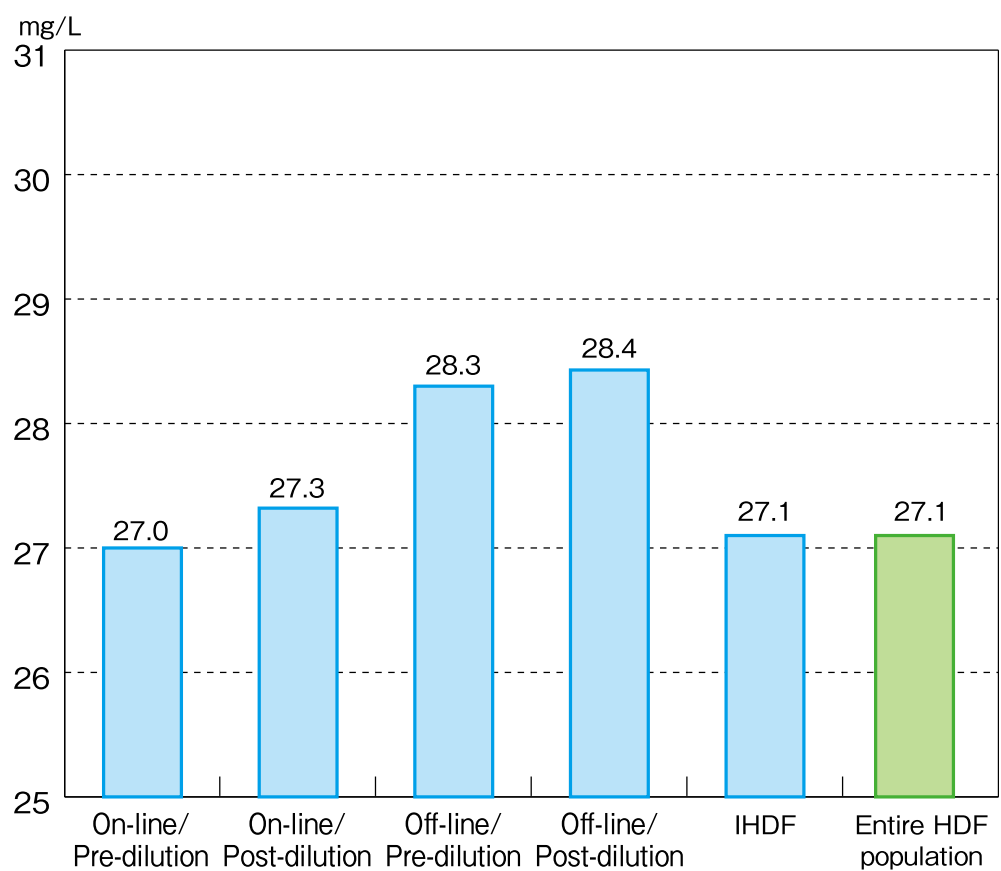

Fig. 40 Mean Pre-dialysis $\beta 2-M G$ concentration in HDF patients, by HDF modality, 2017. $\beta 2-M G$ beta2-microglobulin, HDF hemodiafiltration, IHDF intermittent infusion hemodiafiltraiton

patient/year (1 time/46.2 patients/month) and that the rate tends to decrease as the PD vintage becomes longer (Fig. 48, Additional file 48: Table S48).

The number of patients who responded to the questions regarding $\beta 2-\mathrm{MG}$ level and PD vintage was 3561. Observation of the data by $\mathrm{PD}$ vintage indicates that the lowest mean pre-dialysis $\beta 2$-MG level was $19.68 \mathrm{mg} / \mathrm{L}$ for those with a PD vintage of under 1 year and that there was an increasing trend as the PD vintage increased. The mean $\beta 2-$ MG level for patients who indicated their PD vintage was $26.97 \mathrm{mg} / \mathrm{L}$ (Fig. 49, Additional file 49: Table S49).

\section{Chapter 7: vascular access}

A total of 274,382 patients responded to the questions regarding the type of vascular access, age, and sex. The percentages of those utilizing arteriovenous fistula (AVF) were $91.5 \%$ for males and $84.6 \%$ for females. For both males and females, the percentage of those utilizing AVF
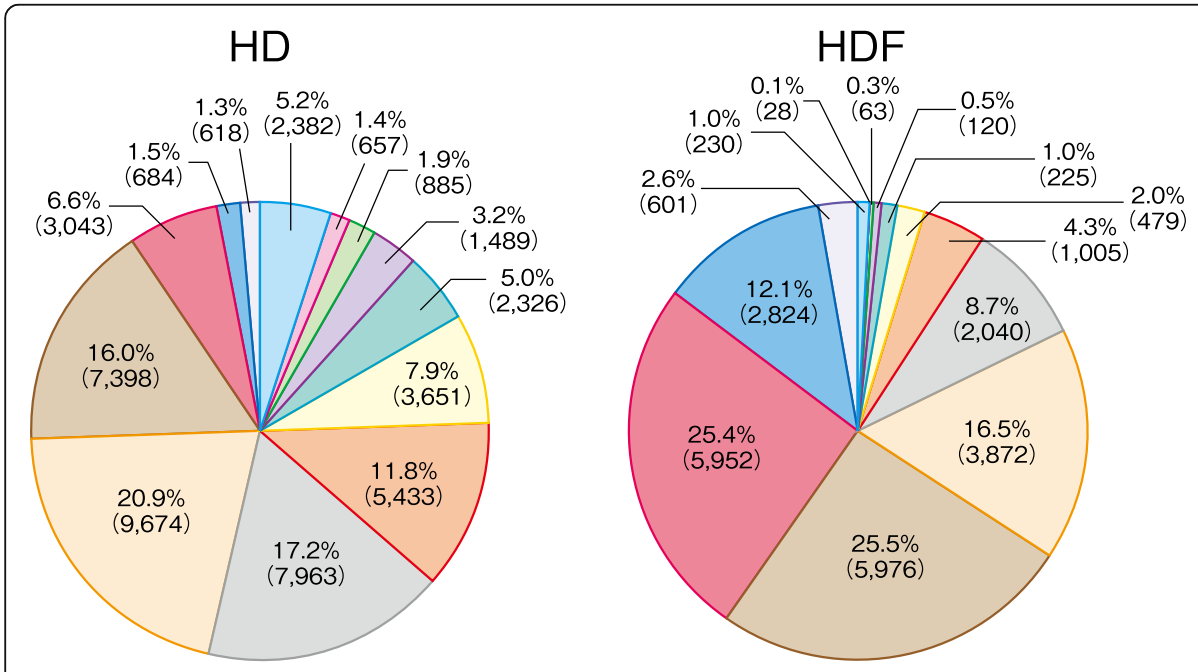

$\beta 2-\mathrm{MG}$ removal rate(\%)
$\square<30$
$\square 30 \leq,<35$
$\square 35 \leq,<40$
$\square 40 \leq,<45$
$\square 45 \leq,<50$
$\square 50 \leq,<55$
$\square 55 \leq,<60$
$\square 60 \leq,<65$
$\square 65 \leq,<70$
$\square 70 \leq,<75$
$\square 75 \leq,<80$
$\square 80 \leq,<85$
$\square 85 \leq$

Fig. 41 HD HDF patient distribution, by $\beta 2-M G$ removal rate, 2017. $\beta 2-M G$ beta2-microglobulin, HD hemodialysis, HDF hemodiafiltration 


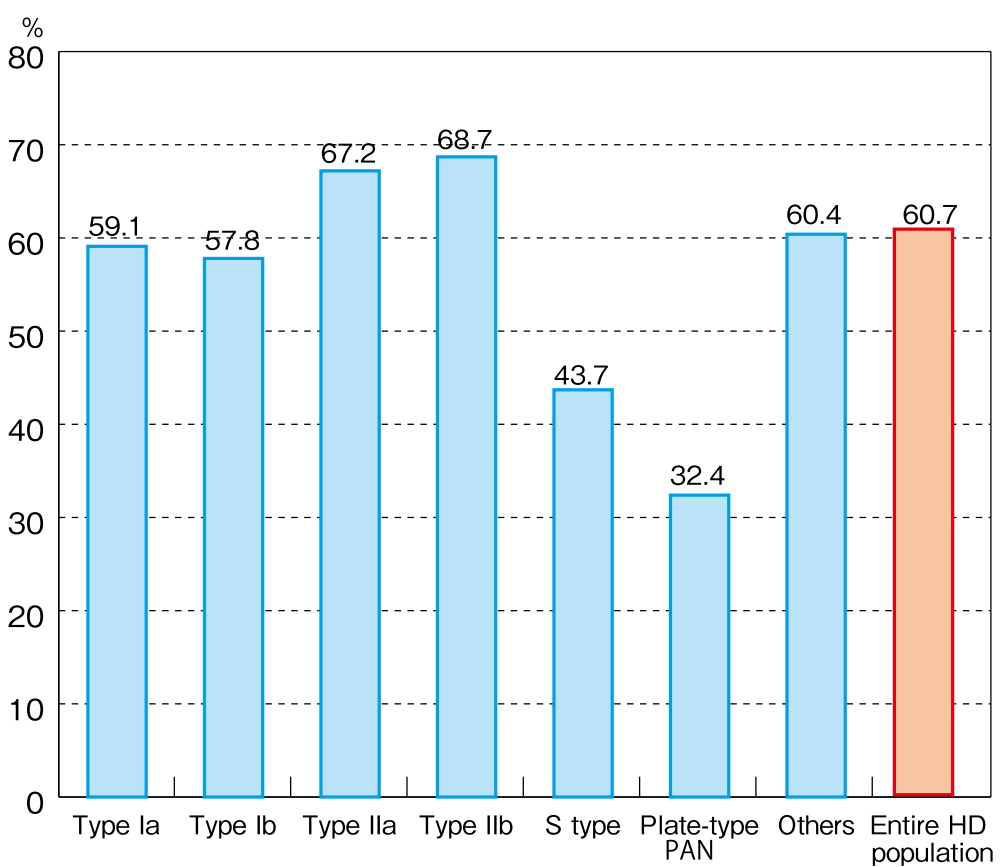

Fig. 42 Mean $\beta 2-M G$ removal rate, in HD patients by dialyzer category, 2017. $\beta 2-M G$ beta2-microglobulin, HD hemodialysis, HDF hemodiafiltration, PAN polyacrylonitrile

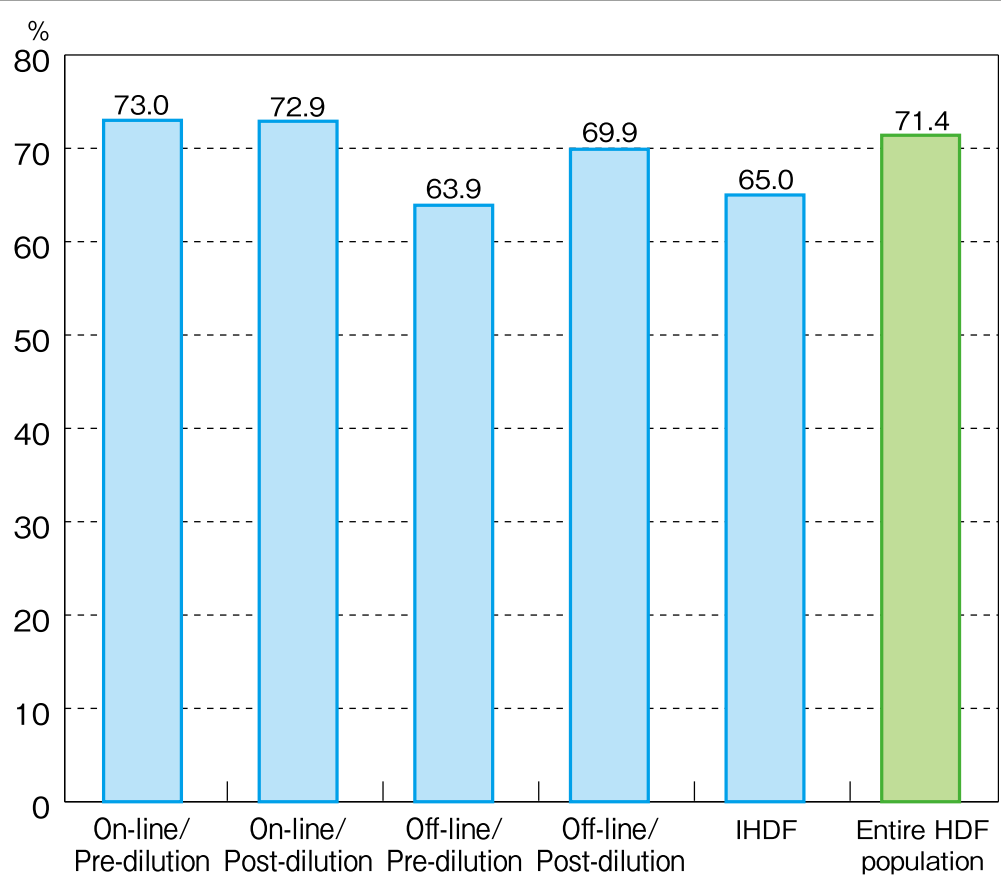

Fig. 43 Mean $\beta 2-M G$ removal rate in HDF patients, by HDF modality, 2017. $\beta 2-M G$ beta2-microglobulin, HDF hemodiafiltration, IHDF intermittent infusion hemodiafiltraiton 


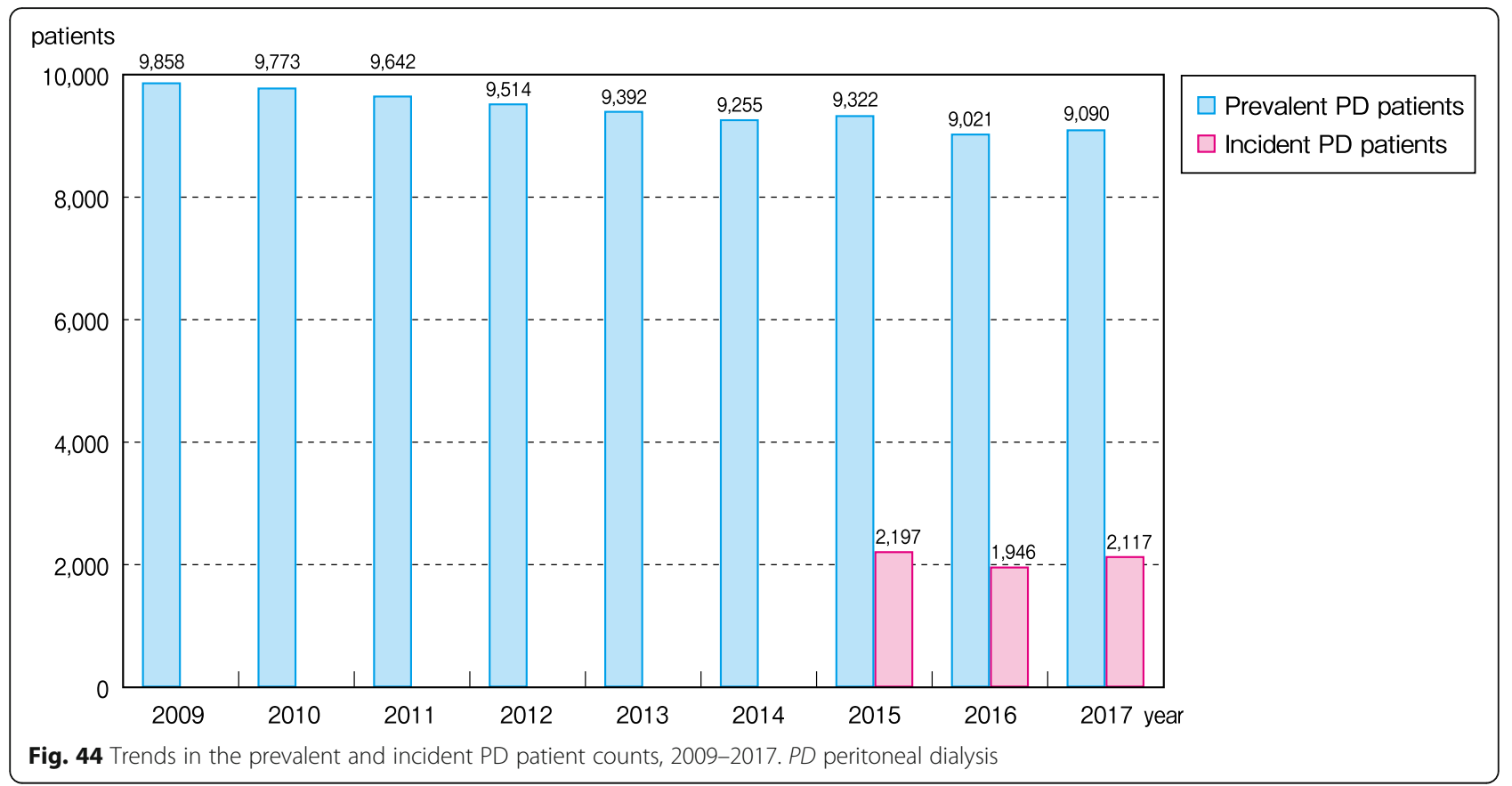

tended to decrease as age increased. The percentages of those utilizing arteriovenous graft (AVG) were $5.5 \%$ for males and $10.6 \%$ for females. The percentages of those utilizing superficialized arteries were $1.6 \%$ for males and $2.0 \%$ for females, and the data indicated that there is a tendency for the percentage of both males and females to increase by aging. The percentages of patients utilizing cuffed central venous catheters $(\mathrm{CVC})$ were $0.9 \%$ for males and $2.2 \%$ for females (Fig. 50, Additional file 50: Table S50).
A total of 274,243 patients responded to the questions regarding the type of vascular access, dialysis vintage, and sex. The percentage of those utilizing AVF showed a tendency to decline as dialysis vintage became longer for both males and females with a dialysis vintage of at least 5 years. The percentage of those utilizing AVG, however, showed a tendency to increase for both males and females as the dialysis vintage became longer (Fig. 51, Additional file 51: Table S51).

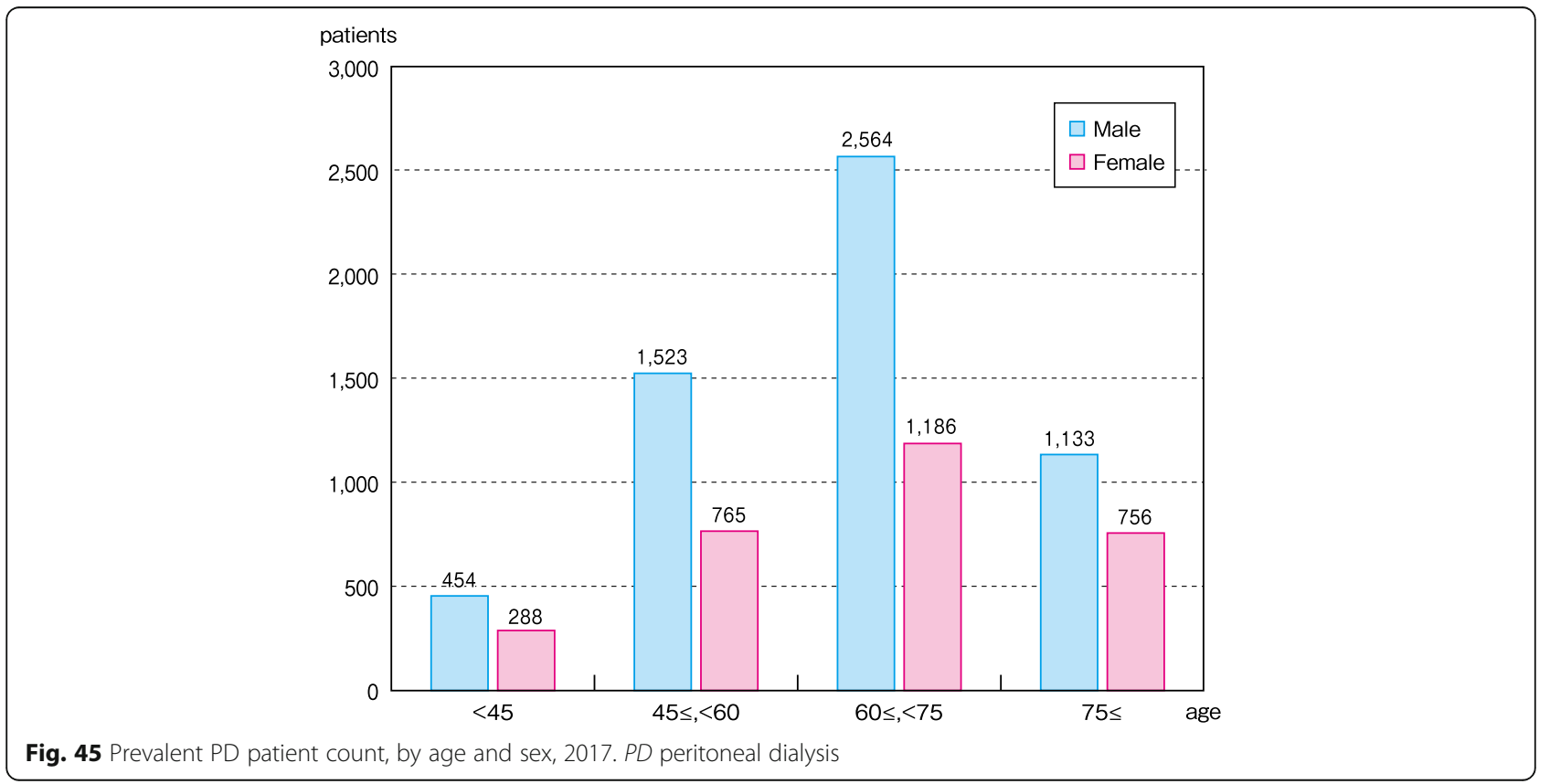




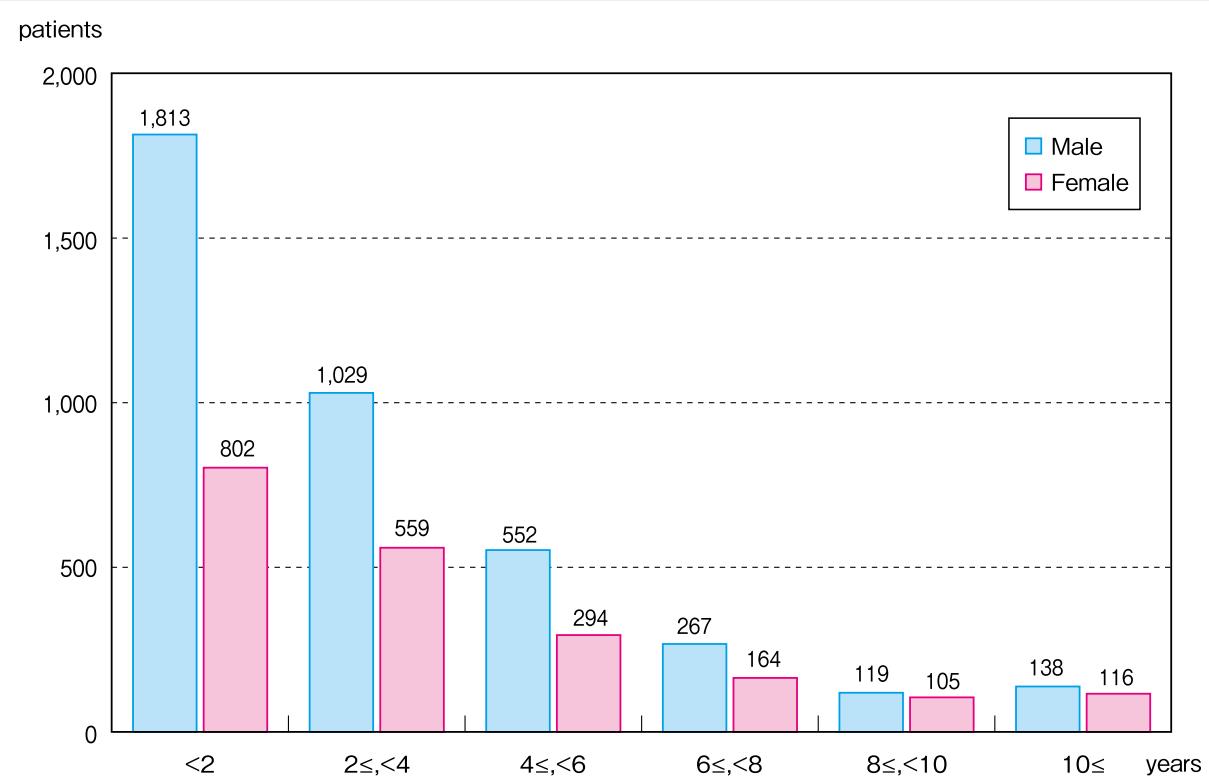

Fig. 46 Prevalent PD patient count, by PD vintage and sex, 2017. PD peritoneal dialysis

\section{Chapter 8: history of carpal tunnel surgery}

A total of 226,791 patients responded to the questions regarding history of carpal tunnel surgery, dialysis vintage, and sex (males: 147,041, females: 79,750). A higher percentage of females indicated that they had a history of carpal tunnel surgery than males (males: 2.9\%, females: $5.5 \%$ ). For both males and females, the percentage of those with a history of carpal tunnel surgery increased as the dialysis vintage became longer, with $62.4 \%$ of the males and $67.5 \%$ of the females with a dialysis vintage of at least 40 years reporting a history of carpal tunnel surgery (Fig. 52, Additional file 52: Table S52).

A total of 58,686 patients responded to the questions regarding history of carpal tunnel surgery and $\beta 2-M G$ removal rate. $\beta 2-\mathrm{MG}$ removal rate was calculated as previously addressed in Chapter 5, 2-6.

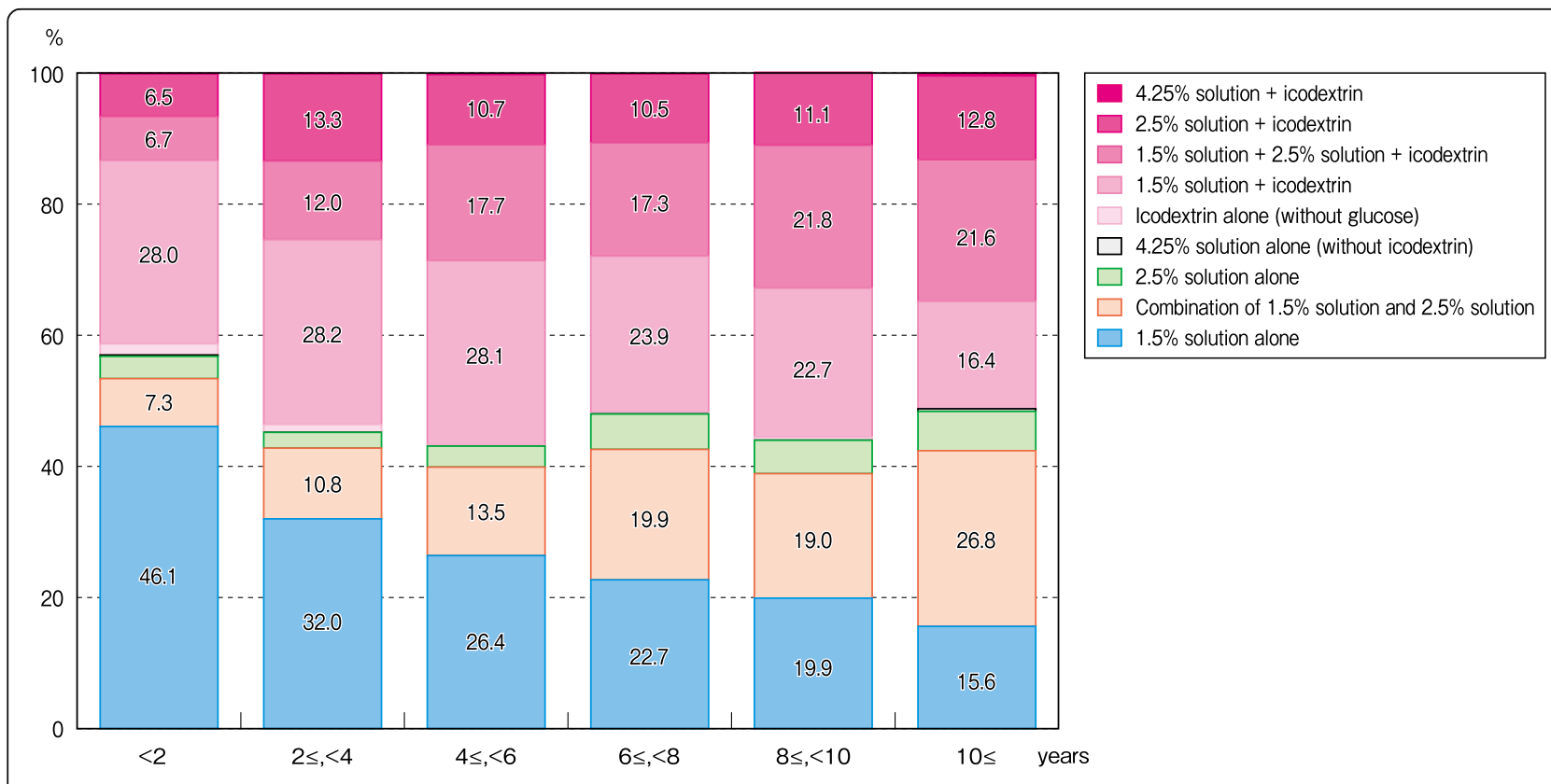

Fig. 47 Prevalent PD patient distribution, by type of PD fluid and PD vintage, 2017. PD peritoneal dialysis 


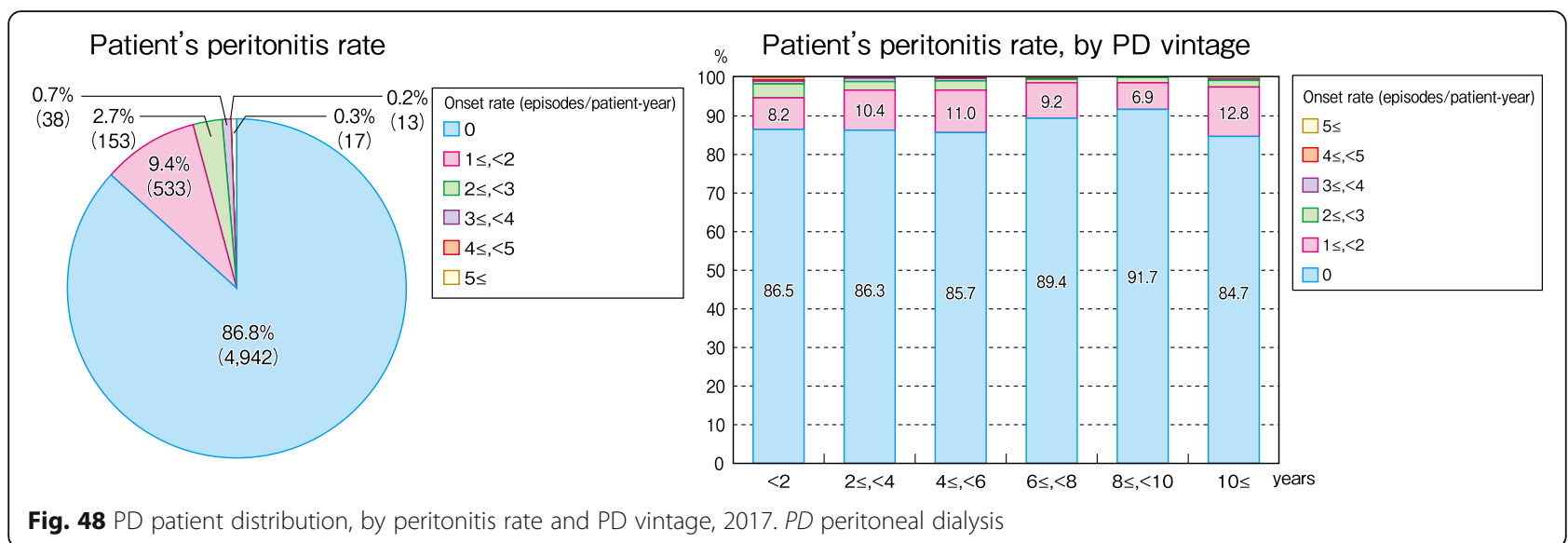

The removal rate of $\beta 2$-MG was $68.1 \pm 13.4 \%$ in the patients with carpal tunnel surgery and $64.0 \pm 15.3 \%$ in the patients without the history. It suggested that those with a history of carpal tunnel surgery undergo treatment with a higher $\beta 2-\mathrm{MG}$ removal rate (Fig. 53, Additional file 53: Table S53).

\section{Chapter 9: hospitalization and cause for hospitalization}

In the 2017 survey, hospitalization, which is an important outcome in the epidemiology of dialysis patients, was investigated. A total of 237,931 patients $(154,171$ males, 83,760 females) responded to the questions regarding hospitalization during 2017, age and sex. The percentages of those who had been hospitalized were
$40.3 \%$ for males and $42.1 \%$ for females. Hospitalizations tended to increase as age increased for both males and females, with high percentages among those aged 75 years and older (males: 46.7\%, females: 50.1\%; Fig. 54, Additional file 54: Table S54).

A total of 237,784 patients $(154,070$ males, 83,714 females) responded to the questions regarding hospitalization during 2017, dialysis vintage, and sex. Hospitalization of the patients were high soon after the start of dialysis (under 5 years) for both males and females (males: $41.9 \%$, females: $44.6 \%$ ) and that they tended to gradually decrease thereafter. The percentages of those with an extremely long dialysis vintage of 30 or more years who were hospitalized increased, reaching almost $40 \%$ for

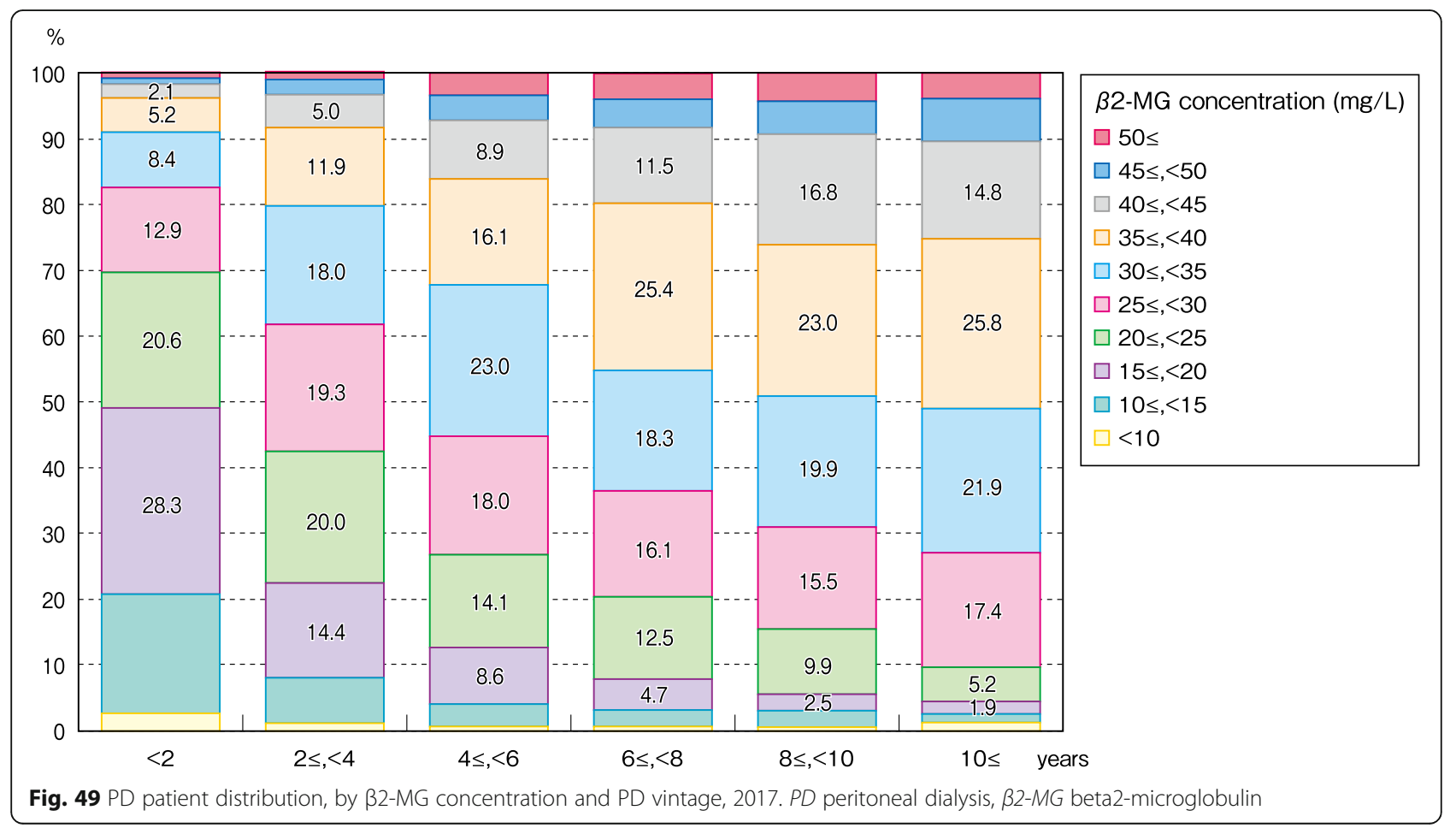




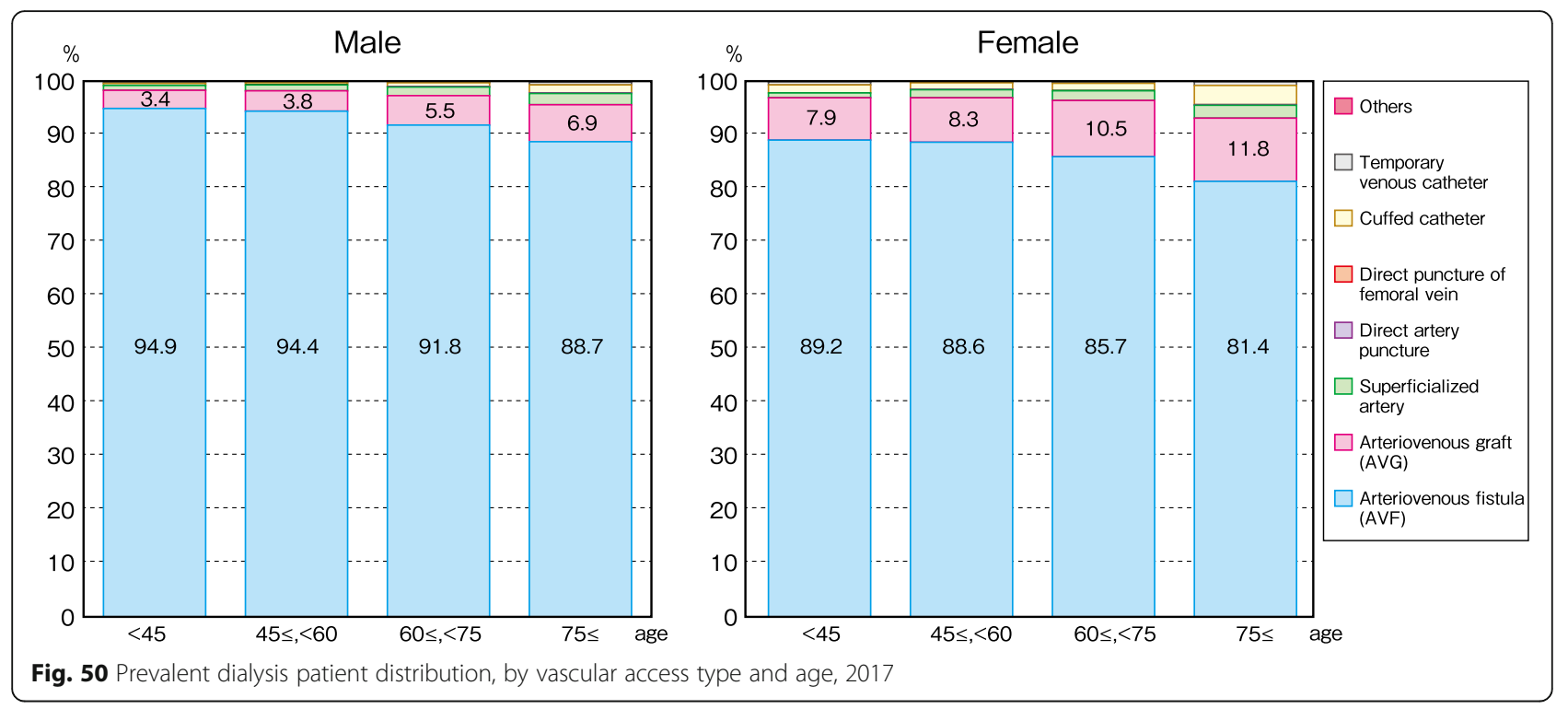

both males and females (Fig. 55, Additional file 55: Table S55).

A total of 237,932 patients $(154,171$ males, 83,761 females) responded to the questions regarding hospitalization during 2017, treatment modalities, and sex. The percentage of those hospitalized was highest among both males and females undergoing PD (males: $60.2 \%$, females: $55.1 \%$ ). Compared to the percentage of those hospitalized for facility hemodialysis (males: $41.4 \%$, females: $43.5 \%$ ), the percentage of those hospitalized for HDF tended to be lower (males: $36.0 \%$, females: $37.6 \%$; Fig. 56, Additional file 56: Table S56).
Of the patients who reported having been hospitalized during 2017, 89,748 (57,331 males, 32,417 females) responded to the questions regarding the cause for hospitalization and sex. As each respondent was able to indicate up to three "causes for hospitalization," the total is not $100 \%$. After excluding "other," the most common causes for hospitalization of males were "cardiac disease" (24.0\%), followed by "vascular access-related" (23.5\%), "infectious disease" (11.3\%), and "orthopedic disease" (8.2\%). After excluding "other," the most common causes for hospitalization of females were "vascular access-related" (27.9\%), followed by "cardiac disease" (17.5\%), "orthopedic

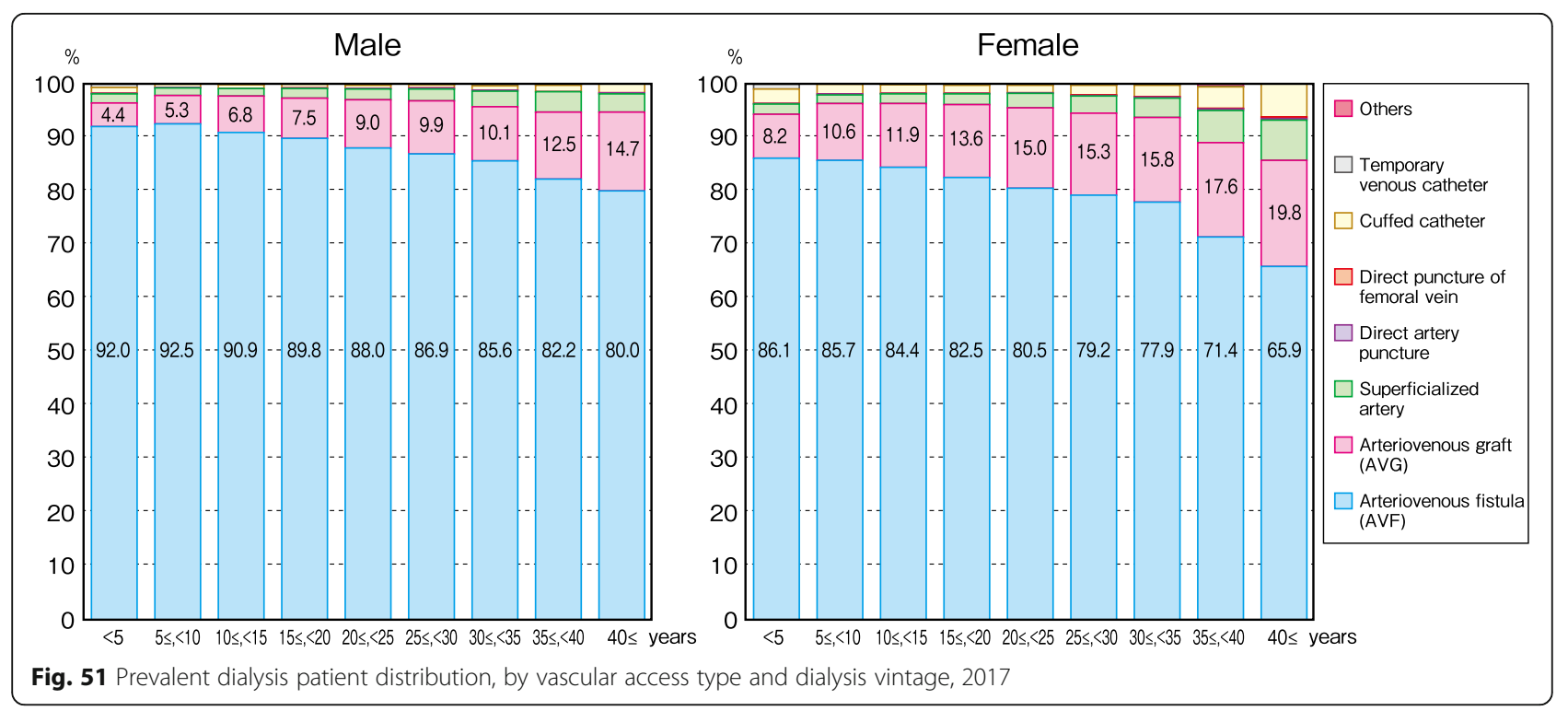




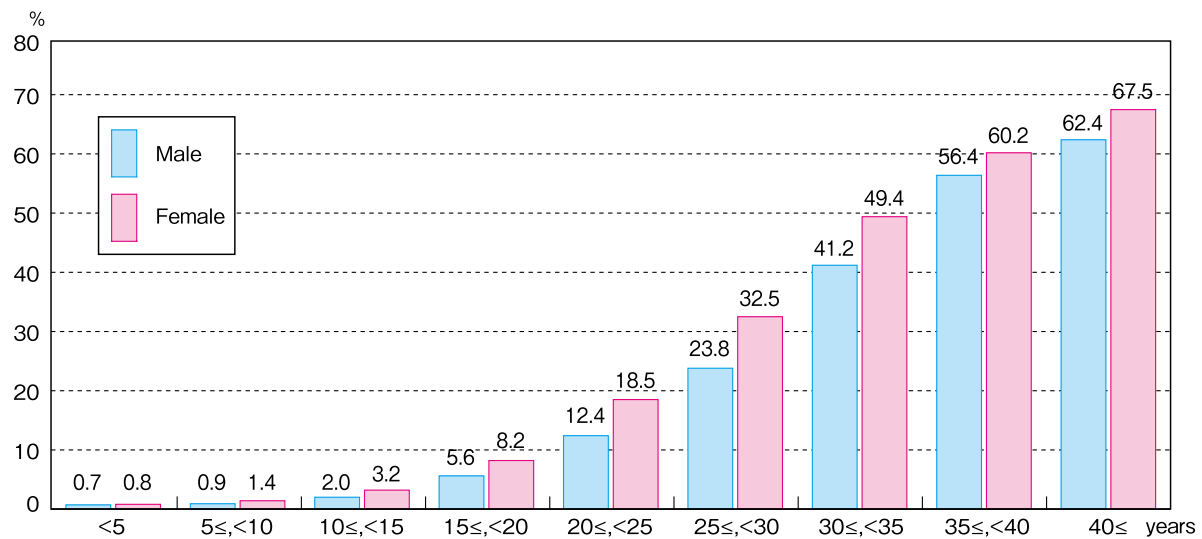

Fig. 52 Distribution of the prevalent patients with the history of carpal tunnel surgery, by dialysis vintage and sex, 2017

disease" (12.6\%), and "infectious disease" (10.4\%) (Fig. 57, Additional file 57: Table S57).

Of those patients who responded that they had been hospitalized during 2017, 89,747 responded to the questions regarding the cause for hospitalization and age. The most common cause for hospitalization, after excluding "other," was "vascular access-related" at all age groups $(25.1 \%$ overall). This was followed overall by "cardiac disease" (21.7\%), "infectious disease" (11.0\%), and "orthopedic disease" (9.8\%). The percentage of those hospitalized for "orthopedic disease" tended to increase as age increased (Fig. 58, Additional file 58: Table S58).

A total of 89,748 patients responded to the questions regarding the cause for hospitalization during 2017 and the treatment modality. After excluding "other," the most common cause for hospitalization for facility hemodialysis patients was "vascular access-related" (26.0\%). This was followed by "cardiac disease" (21.2\%), "infectious disease" (10.7\%), and "orthopedic disease" (9.6\%). After excluding "other," the most common cause for hospitalization for HDF patients was "vascular access-related" (24.6\%). This was followed by "cardiac disease" (24.0\%), "orthopedic disease" (10.9\%), and "infectious disease" (10.0\%). After excluding "other," the most common cause for hospitalization for PD patients was "infectious disease" (24.2\%), followed by "cardiac disease" (15.1\%). As there were only HDF patients, their data is not shown in the figure (Fig. 59, Additional file 59: Table S59).

\section{Conclusion}

The overview of the results of the 2017 JRDR indicates that the number of chronic dialysis patients and the number of dialysis facilities in Japan are still increasing. However, the rate of increase is gradually slowing. No change was observed in the primary diseases of incident patients and patients at the end of the year, with diabetes at number one. However, the percentage of incident patients with diabetes has remained at peak level for several years. HDF treatment, which increased rapidly as a result of the 2017 revision to the medical reimbursement system, increased even further and accounted for $28.4 \%$ of all dialysis patients. Although the number of PD patients and home hemodialysis patients increased slightly over 2016, the rate of home dialysis for both remains lowest in the world, at $2.9 \%$.

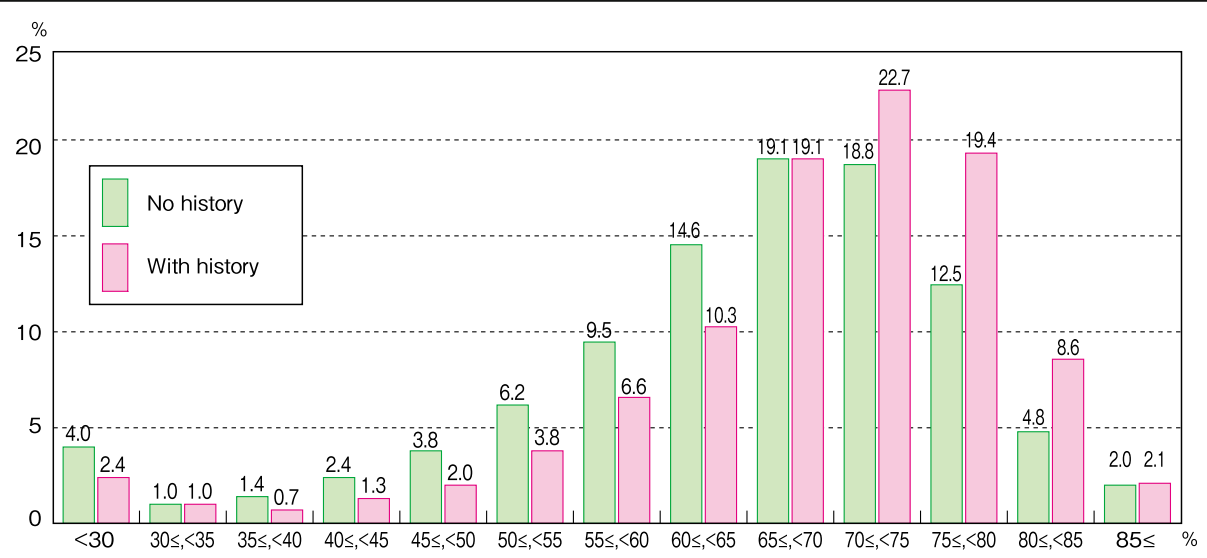

Fig. 53 Prevalent dialysis patient distribution, by history of carpal tunnel surgery and $\beta 2$-MG removal rate, 2017. $\beta 2-M G$ beta2-microglobulin 


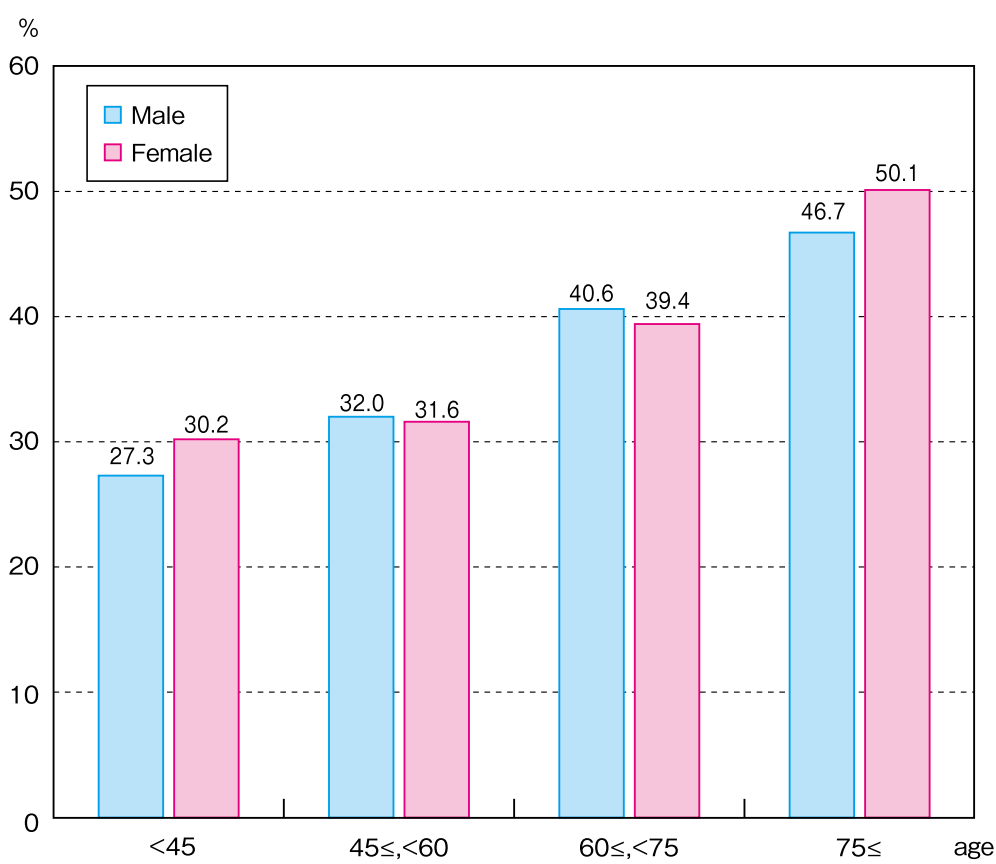

Fig. 54 Distribution of the prevalent dialysis patient with hospitalization, by age and sex, 2017

In the 2017 survey, a detailed investigation of dialysis prescriptions was conducted, as was done in 2008. One characteristic feature of dialysis prescriptions in HD and HDF in Japan have been rather protein permeable than the rest of the world. JRDR has published important reports about the advantages of this characteristic on patient survival [14-16]. We expect to release further information on dialysis prescriptions after further analysis of the results of the 2017 survey. Hospitalization and its reason which are important outcomes as well as a mortality in clinical epidemiology also surveyed in the 2017 JRDR survey. We will analyze their relationship between therapeutic indicators and hospitalization, and we should provide the valuable knowledge to improve the quality of dialysis treatment to the world.

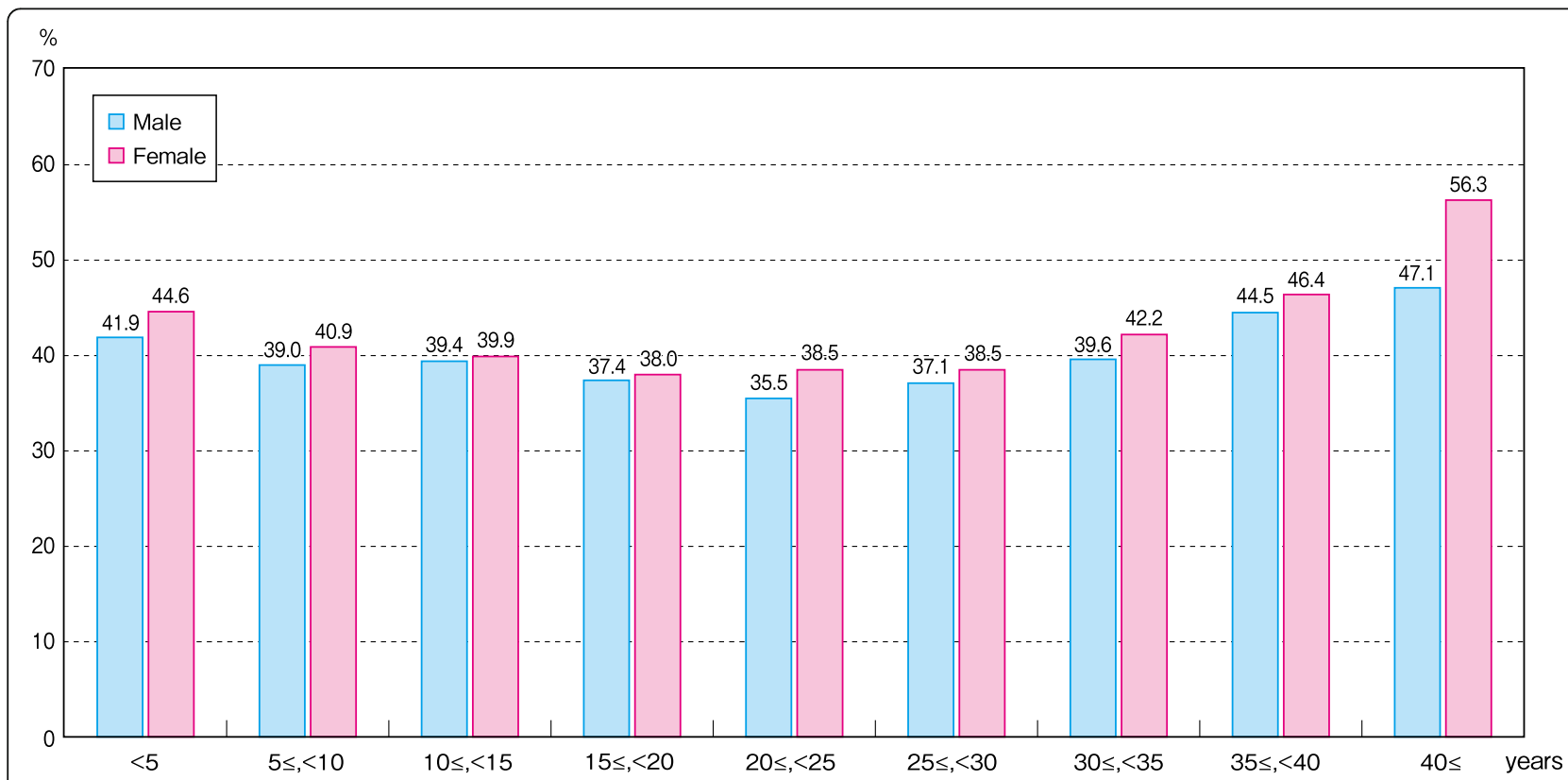

Fig. 55 Distribution of the prevalent dialysis patient with hospitalization, by dialysis vintage and sex, 2017 


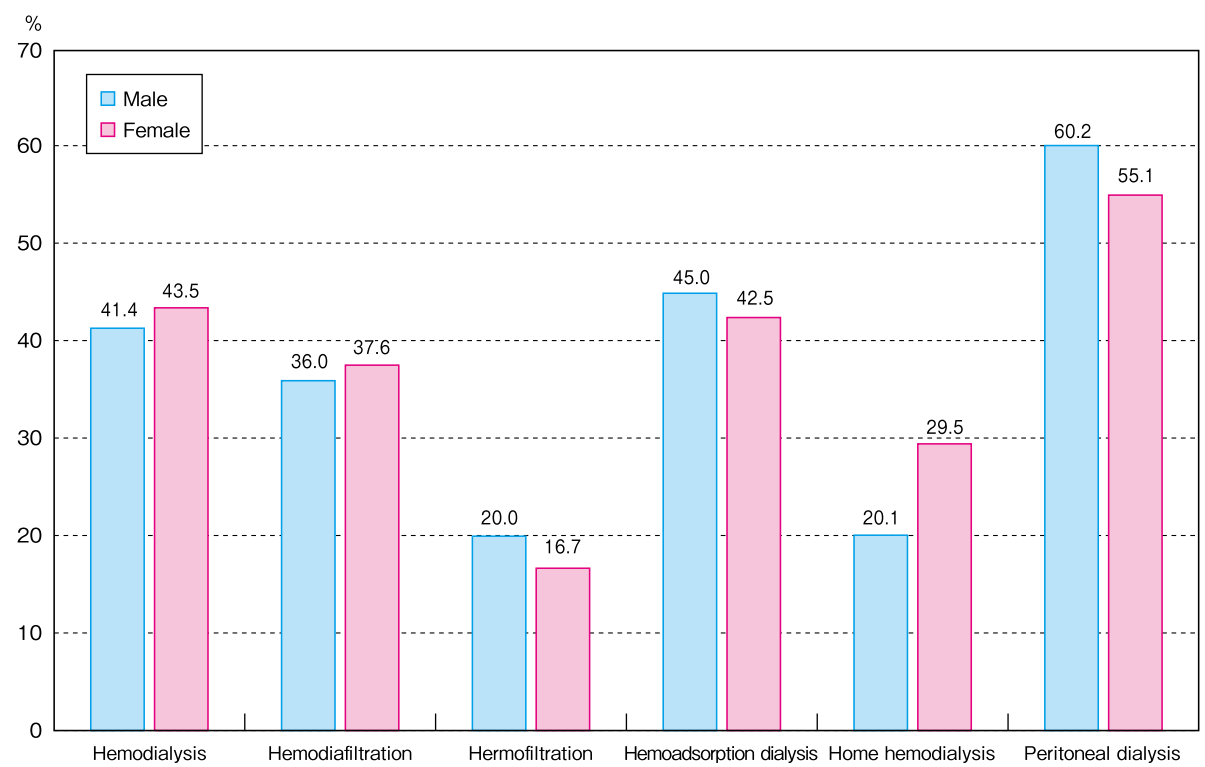

Fig. 56 Distribution of the prevalent dialysis patient with hospitalization, by dialysis modality and sex, 2017

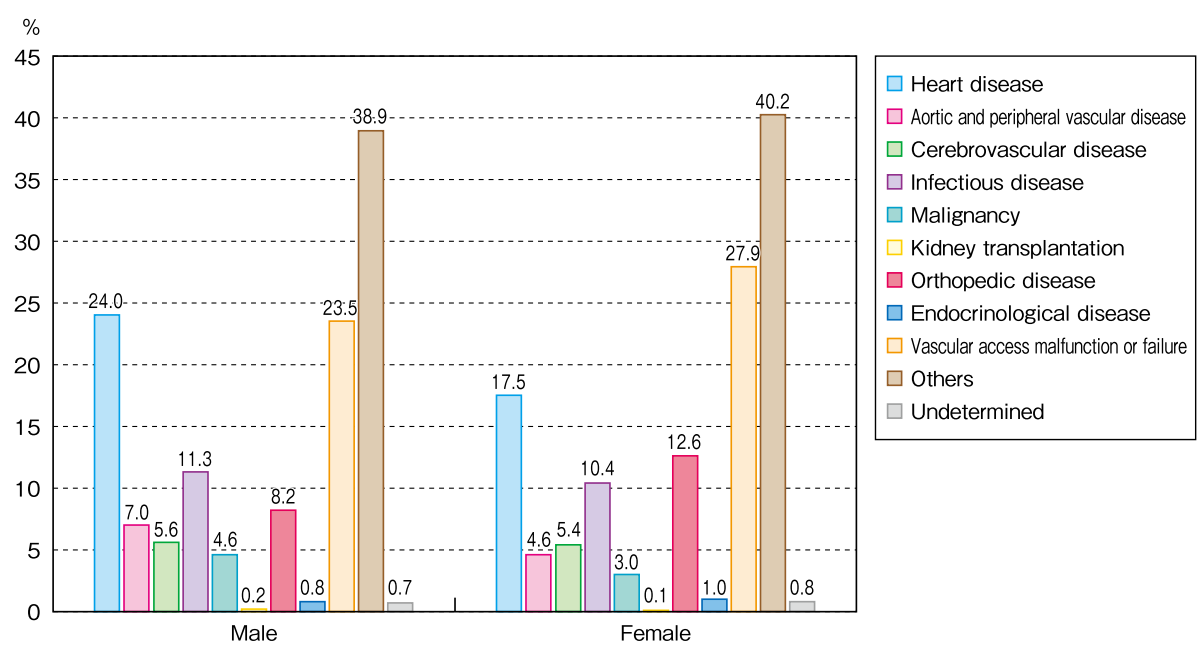

Fig. 57 Distribution of the prevalent dialysis patient with hospitalization, by cause of hospitalization and sex, 2017 

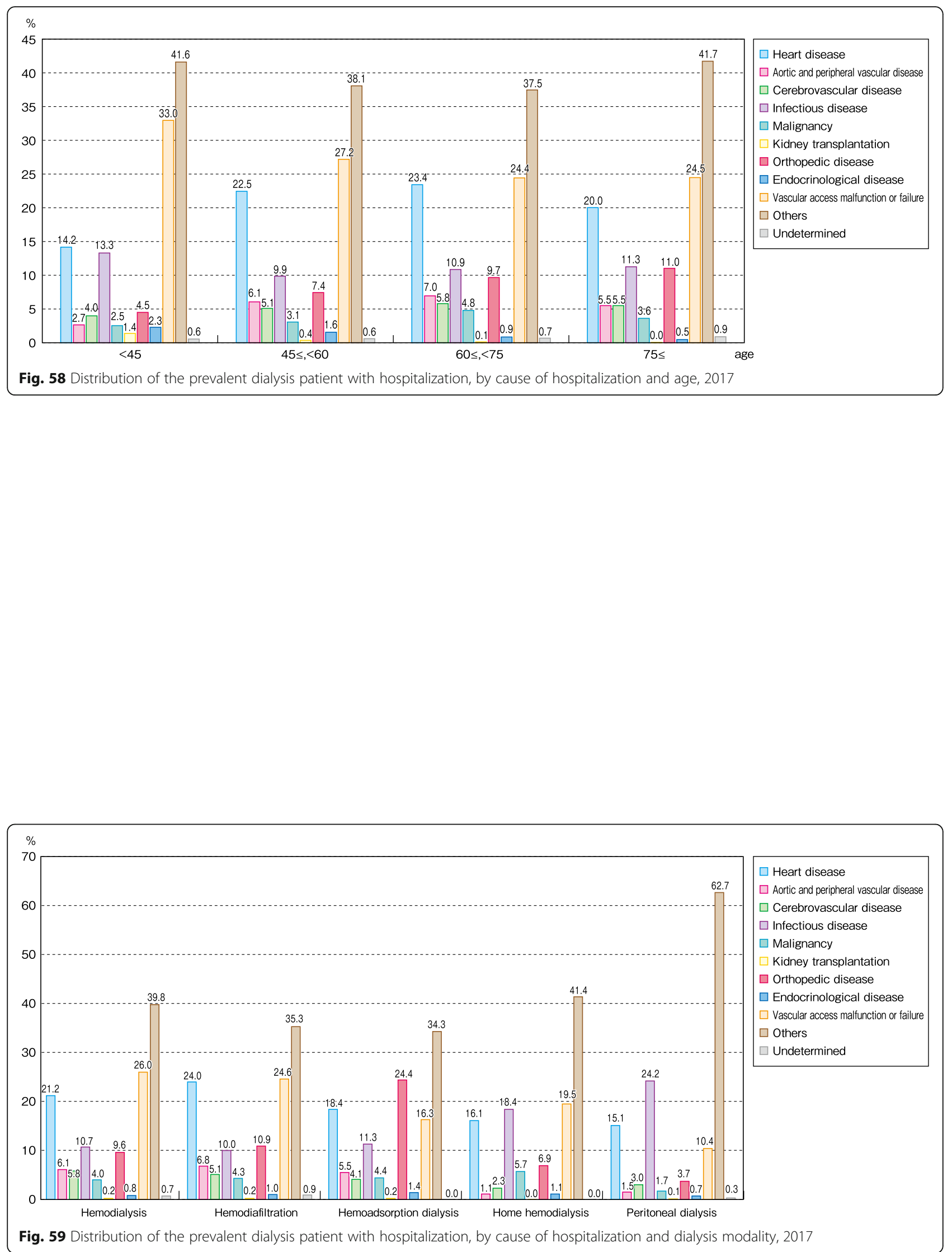


\section{Supplementary information}

Supplementary information accompanies this paper at https://doi.org/10 1186/s41100-019-0248-1

Additional file 1: Table S1. Trends in the prevalent dialysis patient count, 1968-2017 and the adjusted prevalent dialysis patient count (pmp), 1983-2017

Additional file 2: Table S2. Trends in the incident and deceased dialysis patient count, 1983-2017

Additional file 3: Table S3. Prevalent dialysis patient distribution, by age and sex, 2017

Additional file 4: Table S4. Trend in the average age of the prevalent dialysis patients, 1983-2017

Additional file 5: Table S5. Prevalent dialysis patient count, by age, 1982-2017

Additional file 6: Table S6. Prevalent dialysis patient count, by dialysis vintage and sex, 2017

Additional file 7: Table S7. Prevalent dialysis patient count, by dialysis vintage, 1988-2017

Additional file 8: Table S8. Prevalent dialysis patient distribution, by primary disease and sex, 2017

Additional file 9: Table S9. Trends in major primary diseases of the prevalent dialysis patients, 1983-2017

Additional file 10: Table S10. Deceased dialysis patient distribution, by cause of death and sex, 2017

Additional file 11: Table S11. Trends in major causes of death, 19832017

Additional file 12: Table S12. Trend in the annual crude death rate, 1983-2017

Additional file 13: Table S13. Incident dialysis patient distribution, by age and sex, 2017

Additional file 14: Table S14. Trend in the average age of the incident dialysis patients, 1983-2017

Additional file 15: Table S15. Incident dialysis patient distribution, by primary disease and sex, 2017

Additional file 16: Table S16. Trends in major primary diseases of the incident dialysis patients, 1983-2017

Additional file 17: Table S17. Incident dialysis patient distribution, by cause of death and sex, 2017

Additional file 18: Table S18. Trends in major causes of death during the incident year, 1990-2017

Additional file 19: Table S19. Facility distribution, by ETmeasurement frequency and ETconcentration, 2017

Additional file 20: Table S20. Trends in ET measurement frequency and ET concentration, 2006-2017

Additional file 21: Table S21. Facility distribution, by TVC

measurement frequency and TVC, 2017

Additional file 22: Table S22. Trends in TVC measurement frequency and TVC, 2006-2017

Additional file 23: Table S23. Facility distribution, by ET concentration and TVC, 2017

Additional file 24: Table S24. Trends in facility distribution for achieving UPD and standard dialysis fluid, 2009-2017

Additional file 25: Table S25. Facility distribution, by source of dialysis water

Additional file 26: Table S26. Facility distribution, by measurement frequency for residual chlorine and measurement method

Additional file 27: Table S27. Facility distribution, by awareness of the JSDT standard for chemical contaminants and measurement frequency

Additional file 28: Table S28. HD • HDF patient count, by dialysis

modality, 2009-2017
Additional file 29: Table S29. HD HDF patient distribution, by age and sex, 2017

Additional file 30: Table S30. HD HDF patient distribution, by dialysis vintage and sex, 2017

Additional file 31: Table S31. HD HDF patient distribution, by membrane material, 2017

Additional file 32: Table S32. HDF patient distribution, by HDF modality and membrane material, 2017

Additional file 33: Table S33. HD HDF patient distribution, by dialyzer category, 2017

Additional file 34: Table S34. HD HDF patient distribution, by membrane surface area $\left(\mathrm{m}^{2}\right), 2017$

Additional file 35: Table S35. HDF patient distribution, by HDF modality and membrane surface area, 2017

Additional file 36: Table S36. HD HDF patient distribution, by dialysis time, 2017

Additional file 37: Table S37. HD HDF patient distribution, by blood flow rate ( $\mathrm{mL} /$ minutes), 2017

Additional file 38: Table S38. HD HDF patient distribution, by predialysis $\beta 2-M G$ concentration (mg/L), 2017

Additional file 39: Table S39. Mean Pre-dialysis $\beta 2-M G$ concentration in HD patients, by dialyzer category, 2017

Additional file 40: Table S40. Mean Pre-dialysis $\beta 2-M G$ concentration in HDF patients, by HDF modality, 2017

Additional file 41: Table S41. HD . HDF patient distribution, by $\beta 2-M G$ removal rate (\%), 2017

Additional file 42: Table S42. Mean $\beta 2-M G$ removal rate in HD patients, by dialyzer category, 2017

Additional file 43: Table S43. Mean $\beta 2-M G$ removal rate in HDF patients, by HDF modality, 2017

Additional file 44: Table S44. Trends in the prevalent and incident PD patient counts, 2009-2017

Additional file 45: Table S45. Prevalent PD patient count, by age and sex, 2017

Additional file 46: Table S46. Prevalent PD patient count, by PD vintage and sex, 2017

Additional file 47: Table S47. Prevalent PD patient distribution, by type of PD fluid and PD vintage, 2017

Additional file 48: Table S48. PD patient distribution, by peritonitis rate and PD vintage, 2017

Additional file 49: Table S49. PD patient distribution, by pre-dialysis $\beta 2-M G$ concentration and PD vintage, 2017

Additional file 50: Table S50. Prevalent dialysis patient distribution, by vascular access type and age, 2017

Additional file 51: Table S51. Prevalent dialysis patient distribution, by vascular access type and dialysis vintage, 2017

Additional file 52: Table S52. Distribution of the prevalent patients with the history of carpal tunnel surgery, by dialysis vintage and sex, 2017

Additional file 53: Table S53. Prevalent dialysis patient distribution, by history of carpal tunnel surgery and $\beta 2-M G$ removal rate, 2017

Additional file 54: Table S54. Distribution of the prevalent dialysis patient with hospitalization, by age and sex, 2017

Additional file 55: Table S55. Distribution of the prevalent dialysis patient with hospitalization, by dialysis vintage and sex, 2017

Additional file 56: Table S56. Distribution of the prevalent dialysis patient with hospitalization, by dialysis modality and sex, 2017

Additional file 57: Table S57. Distribution of the prevalent dialysis patient with hospitalization, by cause of hospitalization and sex, 2017

Additional file 58: Table S58. Distribution of the prevalent dialysis patient with hospitalization, by cause of hospitalization and age, 2017 
Additional file 59: Table S59. Distribution of the prevalent dialysis patient with hospitalization, by cause of hospitalization and dialysis modality, 2017

\section{Abbreviations}

\%CGR: Percent creatinine generation rate; AFBF: Acetate free biofiltration; APD: Automated peritoneal dialysis; AVF: Arteriovenous fistula; AVG: Arteriovenous graft; CAKUT: Congenital anomalies of the kidney and urinary tract; CD-ROM: Compact disc-read only memory; cfu: Colony forming unit; CKD: Chronic kidney disease; CRP: C-reactive protein; CTA: Cellulose triacetate; CVC: Central venous catheters; D/P Cr ratio: Dialysate/plasma creatinine ratio; EPS: Encapsulating peritoneal sclerosis; ESI: Exit site infection; ESKD: End-stage kidney disease; ET: Endotoxin; ETRF: Endotoxin retentive filter; EVAL: Ethylene vinylalcohol copolymer; HAD: Hemoadsorption dialysis; HD: Hemodialysis; HDF: Hemodiafiltration; HDL-C: High-density-lipoproteincholesterol concentration; HF: Hemofiltration; HHD: Home hemodialysis; HPM: High performance membrane; IHDF: Intermittent infusion hemodiafiltration; JRDR: the JSDT Renal Data Registry; JSDT: Japanese Society for Dialysis Therapy; Kt/N: Index for standardized dialysis dose defined as; K: urea clearance, t: dialysis time, $\mathrm{V}$ : body fluid volume; MEXT: The Ministry of Education, Culture, Sports, Science and Technology; MHLW: The Ministry of Health, Labour and Welfare; nPCR: Normalized protein catabolic rate; PAN: Polyacrylonitrile; PD: Peritoneal dialysis; PEPA: Polyether polymer alloy; PES: Polyethersulfone; PET: Peritoneal equilibration test; PIH: Pregnancyinduced hypertension; PMMA: Polymethylmethacrylate; pmp: Per million population; PS: Polysulfone; PTH: Parathyroid hormone; R2A: Reasoner's Agar No. 2; RRT: Renal replacement therapy (an official journal of JSDT); TAD: Therapeutic apheresis and dialysis (an official journal of JSDT); TGEA: Tryptone glucose extract agar; TVC: Total viable microbial count; UF: Ultrafiltration; UMIN: University hospital Medical Information Network; UMIN-CTR: the UMIN Clinical Trials Registry; UN: Urea N; UPD: Ultrapure dialysis fluid; USB: Universal serial bus; USRDS: the United States Renal Data System; WADDA: Web-based Analysis of Dialysis Data Archives; $32-$ MG: Beta2-microglobulin

\section{Acknowledgments}

We owe the completion of this survey to the efforts of the members of the subcommittee for JRDR Regional Cooperation members mentioned as following, and the staff members of dialysis facilities who participated in the survey and responded to the questionnaires. We would like to express our deepest gratitude to all these people.

Subcommittee for JRDR Regional Cooperation: Kazuyuki Maeno, Tetsuya Kawata, Chikara Oyama, Koji Seino, Toshinobu Sato, Shigeru Sato, Minoru Ito, Junichiro Kazama, Atsushi Ueda, Osamu Saito, Tetsuo Ando, Tomonari Ogawa, Hiroo Kumagai, Makoto Ogura, Hiroyuki Terawaki, Ryoichi Ando, Masaki Abe, Tetsuya Kashiwagi, Chieko Hamada, Yugo Shibagaki, Nobuhito Hirawa, Hisaki Shimada, Yoichi Ishida, Hitoshi Yokoyama, Ryoichi Miyazaki, Mizuya Fukasawa, Yuji Kamijyo, Teppei Matsuoka, Akihiko Kato, Noriko Mori, Yasuhiko Ito, Hirotake Kasuga, Sukenari Koyabu, Tetsuro Arimura, Tetsuya Hashimoto, Masaaki Inaba, Terumasa Hayashi, Tomoyuki Yamakawa, Shinichi Nishi, Akira Fujimori, Tatsuo Yoneda, Shigeo Negi, Akihisa Nakaoka, Takafumi Ito, Hitoshi Sugiyama, Takao Masaki, Yutaka Nitta, Hirofumi Hashimoto, Masahito Yamanaka, Masaharu Kan, Kazumichi Ota, Masahito Tamura, Koji Mitsuiki, Yuji Ikeda, Masaharu Nishikido, Akira Miyata, Tadashi Tomo, Shoichi Fujimoto, Tsuyoshi Nosaki, Yoshinori Oshiro

\section{Authors' contributions}

$\mathrm{KN}, \mathrm{IM}$, and MT finalized the results of the survey and made this manuscript. $\mathrm{SN}, \mathrm{NH}$, and AW designed the survey sheets and made a special program mounted in MS Excel worksheet for the convenience of self-assessment for the dialysis quality of each dialysis facility. T Hase, T Hama, JH, SG, NJ, and MA had the responsibilities on the data analysis. KY and IM had the responsibility on the ethical aspect of the JRDR survey. HN was the president of JSDT in 2017 and checked all the results from the 2017 JRDR survey and approved them to be published. All authors read and approved the final manuscript.

\section{Funding}

There are no funding for the current study. All efforts and costs for the 2015 JRDR survey and making the ADR was totally given by JSDT.

\section{Availability of data and materials}

1. When anyone want to use the data and materials from the current manuscript without modifications, all data and materials are freely available with stating "data from JSDT."

2. When anyone want to use the data and materials from the current manuscript with modifications, any re-calculations or something, they have to state the following sentence in their publication. "The data reported here have been provided by the Japanese Society for Dialysis Therapy (JSDT). The interpretation and reporting of these data are the responsibility of the authors and in no way should be seen as an official policy or interpretation of the JSDT."

\section{Ethics approval and consent to participate}

1. The JSDT registry was approved by the ethical committee of JSDT, the approval no. is 1 .

2. The aims of JSDT Renal Data Registry (JRDR) were well explained for the participated dialysis patients through the dialysis facilities.

3. It does not always need to get the documented approval form from the patients because the all collected data were existing one and there were no new interventions.

4. The original data had been totally anonymized so there are no risks for deteriorating the privacy of the dialysis facilities and the patients.

5. The data presented in the current manuscript does not contain any images, videos, voice recording which might have a risk for identifying an individual.

\section{Consent for publication}

Not applicable.

\section{Competing interests}

The authors declare that they have no competing interests.

Received: 22 October 2019 Accepted: 25 November 2019

Published online: 16 December 2019

\section{References}

1. Nakai S. The history of Japanese Society for Dialysis Therapy Registry. J Jpn Soc Dial Ther. 2010;43:119-52 (in Japanese).

2. Masakane I, Nakai S. Recent trends of chronic dialysis in Japan from the viewpoint of the JSDT Renal Data Registry. J Jpn Soc Dial Ther. 2016;49:2118 (in Japnaese).

3. Ministry of Health, Labour, and Welfare and Ministry of Education, Culture, Sports, Science, and Technology: Ethical Guidelines for Medical and Health Research Involving Human Subjects. http://www.lifescience.mext.go.jp/files/ pdf/n1443_01.pdf, (last accessed 10 Sept 2019).

4. Japanese Society for Dialysis Therapy. http://www.jsdt.or.jp/info/2308.html.

5. Nakai S, Wakai K, Yamagata K, Iseki K, Tsubakihara Y. Prediction of dialysis patients in Japan: based on Japanese Society for Dialysis Therapy Registry. J Jpn Soc Dial Ther. 2012:45:599-613 (in Japanese).

6. Chapter 11: International Comparison, the 2017 USRDS Annual Data Report. Washington: United States Renal Data System, 2017. https://www.usrds. org/2017/view/v2_11.aspx (last accessed 20 July 2018)

7. Nakai $\mathrm{S}$, Iseki K, Itami $\mathrm{N}$, et al. An overview of regular dialysis treatment in Japan (as of December 31, 2010). Ther Apher Dial. 2012;16:483-521.

8. Kawanishi H, Akiba T, Masakane I, Tomo T, Mineshima M, Kawasaki T, et al. Standard on microbiological management of fluids for hemodialysis and related therapies by the Japanese Society for Dialysis Therapy 2008. Ther Apher Dial. 2009:13:161-6.

9. Mineshima M, Kawanishi H, Ase T, Kawasaki T, Tomo T, Nakamoto H. 2016 update Japanese Society for Dialysis Therapy Standard of fluids for hemodialysis and related therapies. Renal Replacement Therapy. 2018;4:15. https://doi.org/10.1186/ s41100-018- 0155-x.

10. Hasegawa T, Nakai S, Masakane I, et al. Dialysis fluid endotoxin level and mortality in maintenance hemodialysis:A nationwide cohort study. Am J Kidney Dis. 2015;65:899-904.

11. Nakai S, Iseki K, Itami N, et al. An overview of regular dialysis treatment in Japan (as of December 31, 2009). Ther Apher Dial. 2012;16:11-53.

12. Watanabe $Y$, Kawanishi H, Suzuki K, et al. Japanese Society for Dialysis Therapy Clinical Guideline for"Maintenance Hemodialysis: Hemodialysis Prescriptions". Ther Apher Dial. 2015;19:67-92. 
13. Li PK, Szeto CC, Piraino B, et al. ISPD peritonitis recommendation: 2016 update on prevention and treatment. Peritoni Dial Int. 2016;36:481-508.

14. Abe M, Hamano T, Wada A, Nakai S, Masakane I, Renal Data Registry Committee JSfDT. Effect of dialyzer membrane materials on survival in chronic hemodialysis patients: Results from the annual survey of the Japanese Nationwide Dialysis Registry. PLoS One. 2017;12:e0184424.

15. Abe M, Hamano T, Wada A, Nakai S, Masakane I. High-performance membrane dialyzers and mortality in hemodialysis patients: a 2-Year cohort study from the annual survey of the Japanese Renal Data Registry. Am J Nephrol. 2017;46:82-92.

16. Kikuchi K, Hamano T, Wada A, Nakai S, Masakane I. Association between predilution on-line hemodiafiltration and survival outcomes in dialysis patients. Kidney Int. 2019;95:929-38.

\section{Publisher's Note}

Springer Nature remains neutral with regard to jurisdictional claims in published maps and institutional affiliations.

Ready to submit your research? Choose BMC and benefit from:

- fast, convenient online submission

- thorough peer review by experienced researchers in your field

- rapid publication on acceptance

- support for research data, including large and complex data types

- gold Open Access which fosters wider collaboration and increased citations

- maximum visibility for your research: over $100 \mathrm{M}$ website views per year

At BMC, research is always in progress.

Learn more biomedcentral.com/submissions 